\title{
ON A NOTION OF MAPS BETWEEN ORBIFOLDS I. FUNCTION SPACES
}

\author{
WEIMIN CHEN
}

\begin{abstract}
This is the first of a series of papers which are devoted to a comprehensive theory of maps between orbifolds. In this paper, we define the maps in the more general context of orbispaces, and establish several basic results concerning the topological structure of the space of such maps. In particular, we show that the space of such maps of $C^{r}$ class between smooth orbifolds has a natural Banach orbifold structure if the domain of the map is compact, generalizing the corresponding result in the manifold case. Motivations and applications of the theory come from string theory and the theory of pseudoholomorphic curves in symplectic orbifolds.
\end{abstract}

\section{INTRODUCTION}

The space of differentiable maps is a fundamental object associated to smooth manifolds. More precisely, given smooth manifolds $M$ and $N$, one considers the set $C^{r}(M, N)$ of $C^{r}$ maps $f: M \rightarrow N$, ie. maps with continuous partial derivatives up to a given order $r$. It is well-known that $C^{r}(M, N)$ has a natural topology, called the Whitney topology, which in the case when $M$ is compact also gives $C^{r}(M, N)$ an infinite dimensional manifold (ie. Banach manifold) structure. A particularly important case is when $M$ is the circle $S^{1}$, in this case the space of maps is called the loop space of $N$. These function spaces are fundamental in many branches of mathematics, including algebraic and differential topology of manifolds, differential geometry and global analysis, as well as mathematical physics.

The purpose of this paper is to formulate a notion of maps between orbifolds, which is given in the more general perspective of orbispaces, such that orbifolds form a category under such maps. Several basic results concerning topological structure of the space of maps were established. In particular, we showed that the space of $C^{r}$-maps between smooth orbifolds $X$, $X^{\prime}$ is a Banach orbifold when $X$ is compact. Based on these structural results, we developed in the sequel [6] a basic machinery for studying homotopy classes of such maps.

Considerations in this work originated from construction of Gromov-Witten invariants of symplectic orbifolds $[10,11]$ and from our attempt to understand some of the mathematical implication of the work of Dixon, Harvey, Vafa and Witten [12] on string theories of orbifolds. While the treatment in $[10,11]$ was ad hoc in nature, the aforementioned structural theorem on mapping spaces provided a solid foundation for the Fredholm theory of pseudoholomorphic curves in symplectic orbifolds, which was more instrumental in dimension 4 in the recent, more geometric application in [7,8]. On the other hand, concerning the orbifold string theories it is worth noting that, with the notion of maps in this paper, the loop space of an

Date: September 12, 2018

Keywords. Orbifold. Mapping space. 2000 Mathematics Subject Classification. Primary 22A22. Secondary 57P99, 58D99, 46T20. 
orbifold gives exactly the configuration space of strings considered by the physicists in [12], where the orbifolds are global quotients $X=Y / G$. Given the fact that spaces of differentiable maps have been involved in many considerations in geometry, topology and mathematical physics, it is our hope that this work will pave the way for similar considerations, especially those originating from mathematical physics, in the orbifold category.

A brief history of orbifolds. The concept of orbifold was first introduced by Satake under the name "V-manifold" in his 1957 paper [31], where orbifolds were perceived as a class of singular spaces which can be modeled locally on a smooth manifold modulo a finite group. The smooth manifold together with the group action is called a local uniformizing system. The purpose of Satake was to demonstrate that the basic differential geometry of smooth manifolds can be suitably extended to this class of singular spaces. The local structure of orbifold, ie. being locally modeled as the quotient of a smooth manifold by a finite group action, was merely used as sort of generalized smooth structure here. This was clearly reflected in the notion of "V-manifold map" introduced by Satake, which was roughly speaking a continuous map with smooth liftings to local uniformizing systems. The basic intuition here is that orbifolds behave very much like smooth manifolds, as long as only $\mathbb{Q}$ coefficients are concerned.

The more popular name "orbifold" was due to Thurston [33], who rediscovered this concept in the late 70's as a useful device in studying geometric structures on 3-manifolds. Here is the basic idea. The quotient manifold $Y / \Gamma$ provides a useful, geometric device for studying a free, proper, and discontinuous action of $\Gamma$ on $Y$. In order to extend this to the case where the action may not be free, one has to allow singularities in the quotient space $Y / \Gamma$. The key issue here, however, is how to recover the action from the quotient space when it is not free. To this end, Thurston introduced the notion of fundamental group of an orbifold, which has the property that when $Y$ is simply-connected, the fundamental group of the orbifold $Y / \Gamma$ is always $\Gamma$, regardless the action is free or not. This is obviously different from the fundamental group of $Y / \Gamma$ regarded as a singular topological space. Thurston's notion of fundamental group proves to be particularly useful in a context where the orbifold arises as the quotient space of a group action.

There are two major sources of orbifolds. One is as the quotient space of either a proper discontinuous action or a compact Lie group action with only finite isotropy subgroups. The other is as a space or a variety with only "mild" singularities (for instance, as those appeared in birational transformation or in degeneration of certain Riemannian metrics), where there is not necessarily a natural global group action involved. Despite the abundance of appearance in the literature, orbifolds have been mainly involved as a useful technical device rather than an object of permanent interest, and have been treated in a rather practical, ad hoc manner, keeping in mind the basic observations of Satake and Thurston. In particular, there has been no comprehensive study or theory on orbifolds (eg. as the one we had on manifolds).

There is a somewhat formal and more contemporary formalism of orbifolds (compared with that in Satake [31] or Thurston [33]), using the categorical language of étale (smooth) topological groupoids (cf. eg. [1]). One of the advantages of this formalism is that canonical, functorial constructions on topological groupoids provided additional tools associated to the orbifolds (these, of course, work for manifolds also, but only become nontrivial when applied to orbifolds). For example, one can associate an orbifold with a classifying space (which 
is the classifying space of the associated étale topological groupoids, and which is unique up to homotopy equivalence), and define topological invariants of orbifolds through the classifying spaces, cf. eg. [15]. Thurston's orbifold fundamental group may be recovered as the fundamental group of the classifying space. This approach is particularly useful in the case of cofunctors, such as cohomologies, fibre bundles, characteristic classes, etc.

String theories on orbifolds. The suggestion of having a comprehensive study of orbifolds (as the one for manifolds) seemed to come first from string theory. In 1985, physicists Dixon, Harvey, Vafa and Witten [12] considered string theories on a compact Calabi-Yau manifold $Y$ equipped with a finite group action of $G$ preserving the Calabi-Yau structure of $Y$ (more generally, $Y$ could be noncompact with the quotient $X=Y / G$ being a compact orbifold). For the purpose of symmetry breaking, it was necessary to not only consider strings $y(t)$ which satisfies a periodic boundary condition, but also to consider those satisfying boundary conditions which are periodic up to the action of $G$ :

$$
y(t+2 \pi)=g \cdot y(t), \text { for some } g \in G
$$

(These more general boundary conditions are called "twisted boundary conditions".) An interesting idea in [12] was to study such string theories on $Y$ as a (closed) string theory on the quotient orbifold $X=Y / G$.

One of the advantages of introducing string theories on orbifolds is that string propagation on an orbifold may be regarded as an arbitrarily good approximation to the string propagation on any of the smooth resolutions of the orbifold. Given such a relation between string theories on a Calabi-Yau orbifold $X$ and any of its crepant resolutions $\tilde{X}$ (if there exists one), the physicists made some remarkable prediction concerning the Euler number $e(\tilde{X})$ of $\tilde{X}$ in terms of the orbifold $X$.

More concretely, in string theories on a smooth manifold, the Euler number of the manifold may be interpreted as twice of the "number of generations" in the physical theory. Extending this to orbifolds, one may simply define the "stringy Euler number" of an orbifold to be twice of the "number of generations" in the physical theory. With this understood, the physicists derived, using the path integral method, the following formula for the "stringy Euler number" of the Calabi-Yau orbifold $X=Y / G$ :

$$
e_{\text {string }}(X)=\frac{1}{|G|} \sum_{h g=g h} e\left(Y^{<g, h>}\right)
$$

where $Y^{<g, h>}$ denotes the common fixed-point set of $g$ and $h$ in $Y$. By the said relation between string theories on $X$ and any of its crepant resolutions $\tilde{X}$, the physicists then obtained the following identity

$$
e(\tilde{X})=\frac{1}{|G|} \sum_{h g=g h} e\left(Y^{<g, h>}\right) .
$$

The right-hand side of (1.2) was later reformulated by Hirzebruch and Höfer [20], and consequently (1.3) becomes

$$
e(\tilde{X})=e(X)+\sum_{(g), g \neq 1} e\left(Y^{g} / Z(g)\right)
$$


where $Y^{g}$ is the fixed-point set of $g$ in $G,(g)$ stands for the conjugacy class of $g$ in $G$ and $Z(g)$ is the centralizer of $g$ in $G$. The advantage of the right-hand side of (1.4) over that of (1.2) is that: (1) it explicitly shows that the "stringy Euler number" $e_{\text {string }}(X)$ of an orbifold $X$ differs in general from the Euler number $e(X),(2)$ the right-hand side of (1.4) can be defined for any orbifold, which is not necessarily of the form $Y / G$ with $G$ finite.

The physicists's prediction (1.3) (or equivalently (1.4)) was soon related to the so-called "McKay correspondence" in mathematics (cf. [27]), and has since stimulated a great deal of interests in this subject. See [30] for a recent survey on McKay correspondence.

Similar predictions may be made on other types of invariants, such as Gromov-Witten invariants, elliptic cohomology, etc., which also have an alleged string theory interpretation (cf. $[34,35,32]$ ). In order to fully explore in this direction of research, it is desirable to develop a comprehensive theory of orbifolds, which would provide a framework within which these "stringy invariants" of orbifolds may be properly interpreted and further studied. The theory of maps between orbifolds developed in this work was motivated by such a desire. It turns out that this theory of maps (1) is amenable to techniques of differential geometry and global analysis on orbifolds as pioneered by Satake, (2) reflects the distinct topological structure of orbifolds as discovered by Thurston, and (3) is consistent to the considerations in string theories of orbifolds by the physicists in [12].

Gromov-Witten invariants of orbifolds. The theory of maps between orbifolds developed in this work originated in the construction of Gromov-Witten invariants of symplectic orbifolds in $[10,11]$.

Recall that Gromov-Witten invariants of a symplectic manifold $(M, \omega)$ come as a certain algebraic count of $J$-holomorphic curves in $M$ for some fixed, $\omega$-tamed almost complex structure $J$. The actual counting of $J$-holomorphic curves goes roughly as follows. One introduces a Banach manifold $B$, which is the space of maps of a certain fixed Sobolev or Hölder type from a Riemann surface into $M$, and a Banach bundle $E \rightarrow B$ such that the set of $J$-holomorphic curves in question is given as the zero set of a Fredholm section $s: B \rightarrow E$. In nice situations, one can show that for a generic choice of $J$ the Fredholm section $s$ intersects transversely with the zero section of $E$, and consequently, as the zero set the moduli space of $J$-holomorphic curves is a smooth manifold whose dimension can be computed via the Riemann-Roch theorem. Furthermore, one can compactify the moduli space using Gromov's compactness theorem, and again in nice situations one can show that the compactification of the moduli space has only codimension at least 2 "boundary" components, thus has a well-defined "fundamental class" in the Banach manifold $B$. In these nice situations, the Gromov-Witten invariants are defined by evaluating certain "universal" cohomology classes on the Banach manifold $B$ against the fundamental class of the compactified moduli space of $J$-holomorphic curves, which turns out to be independent of the choice of the almost complex structure $J$. (For details, see eg. [26].) In any event, such a setup in the Banach manifold framework is the so-called Fredholm theory of pseudoholomorphic curves.

The above construction in the manifold case does not generalize to orbifolds in any obvious way because there is a lack of corresponding theory of maps between orbifolds, and thus no Fredholm theory is available. More concretely, one may regard a $J$-holomorphic curve in a symplectic orbifold as the image of a $J$-holomorphic $V$-manifold map (in the sense of Satake) from an orbifold Riemann surface into the symplectic orbifold. The problem is that 
the corresponding space of $\mathrm{V}$-manifold maps is not known to have any infinite dimensional manifold or orbifold structure. On the other hand, there is also a related but more conceptual issue due to the lack of a good theory of maps (or in a more general sense, due to a lack of comprehensive theory of orbifolds), that is, how to determine whether the constructed Gromov-Witten invariants of symplectic orbifolds (no matter how technically the construction was done) actually give the corresponding "stringy invariants" in the orbifold string theory [12].

The problem of orbifold Gromov-Witten theory was solved in [10, 11] at the technical level. A crucial step in the construction is to add an additional piece of data to a $\mathrm{V}$-manifold map, which is an isomorphism class of pull-back bundles by the V-manifold map. The secret behind this is that one needs to specify a deformation type for each $J$-holomorphic curve in order to "count" the J-holomorphic curves properly. The resulting Gromov-Witten invariants turn out to be a certain algebraic count of $J$-holomorphic curves together with a specified deformation type in the orbifold. Formally, one would have defined the GromovWitten invariants in $[10,11]$ by working with the space of pairs $(f, \xi)$, where $f$ is a $\mathrm{V}$-manifold map and $\xi$ is an isomorphism class of pull-back bundles by $f$. However, no Fredholm theory based on the space of pairs $(f, \xi)$ was available back then, so the construction in $[10,11]$ was carried out in a rather ad hoc manner.

The pair $(f, \xi)$ in the preceding paragraph is a prototype of the maps defined in this paper. In this regard, the theory of maps developed in this work has accomplished the following two goals: (1) providing a necessary foundation for the Fredholm theory of pseudoholomorphic curves in symplectic orbifolds, and (2) providing a mathematical framework for the stringy interpretation of the Gromov-Witten invariants constructed in [10, 11]. In particular, the construction of Gromov-Witten invariants of symplectic orbifolds may be done in the same line as in the smooth case, and the one in $[10,11]$ can be substantially simplified.

In dimension 4, Gromov's pseudoholomorphic curve theory has had more geometric applications (cf. eg. [26]), where the manifold structure of the corresponding moduli space of pseudoholomorphic curves played a fundamental role. (To the contrary, in the problem of counting pseudoholomorphic curves one only needs a well-defined fundamental class of the moduli space.) In order to apply similar ideas to a situation where quotient singularities are present, a Fredholm theory in the orbifold context must be in place first. For a theory of pseudoholomorphic curves in symplectic 4-orbifolds and some related applications, see the recent survey [9], and for more details, see [7, 8].

Extension of the equivariant category. Define the "equivariant category" as follows: the objects are pairs $(Y, G)$, where $Y$ is a smooth manifold equipped with a smooth action of a finite group $G$, and the morphisms from $(Y, G)$ to $\left(Y^{\prime}, G^{\prime}\right)$ are pairs $(f, \rho)$, where $f: Y \rightarrow Y^{\prime}$ is a differentiable map and $\rho: G \rightarrow G^{\prime}$ is a homomorphism, such that $f$ is $\rho$-equivariant, ie. $f(g \cdot y)=\rho(g) \cdot f(y)$ for any $y \in Y$ and $g \in G$. Then the category of orbifolds, where the objects are smooth orbifolds and the morphisms are differentiable maps between orbifolds defined in this paper, may be regarded as an extension of the equivariant category in the following sense.

Given any two objects $(Y, G)$ and $\left(Y^{\prime}, G^{\prime}\right)$ of the equivariant category, let $X=Y / G$ and $X^{\prime}=Y^{\prime} / G^{\prime}$ be the corresponding orbifolds. Let $\left[(Y, G) ;\left(Y^{\prime}, G^{\prime}\right)\right]$ denote the set of morphisms $(f, \rho):(Y, G) \rightarrow\left(Y^{\prime}, G^{\prime}\right)$, and let $\left[X ; X^{\prime}\right]$ denote the set of morphisms $\Phi: X \rightarrow X^{\prime}$. Then 
each $(f, \rho) \in\left[(Y, G) ;\left(Y^{\prime}, G^{\prime}\right)\right]$ defines a differentiable map $\Phi \in\left[X ; X^{\prime}\right]:(f, \rho) \mapsto \Phi$, which induces an identification as a subset (cf. Lemma 3.1.2)

$$
\left[(Y, G) ;\left(Y^{\prime}, G^{\prime}\right)\right] / G^{\prime} \subset\left[X ; X^{\prime}\right]
$$

where the action of $G^{\prime}$ on $\left[(Y, G) ;\left(Y^{\prime}, G^{\prime}\right)\right]$ is given by

$$
g^{\prime} \cdot(f, \rho)=\left(g^{\prime} \circ f, A d\left(g^{\prime}\right) \circ \rho\right), \forall g^{\prime} \in G^{\prime},(f, \rho) \in\left[(Y, G) ;\left(Y^{\prime}, G^{\prime}\right)\right] .
$$

Moreover, each $\Phi: X \rightarrow X^{\prime}$ induces a homomorphism between Thurston's orbifold fundamental groups $\Phi_{*}: \pi_{1}(X) \rightarrow \pi_{1}\left(X^{\prime}\right)$, such that $\Phi$ is defined by a pair $(f, \rho)$ if and only if $\Phi_{*}$ maps the subgroup $\pi_{1}(Y)$ of $\pi_{1}(X)$ into the subgroup $\pi_{1}\left(Y^{\prime}\right)$ of $\pi_{1}\left(X^{\prime}\right)$ (cf. Lemma 2.4.1 in [6]). Finally, for any $r>0$, let $\left[(Y, G) ;\left(Y^{\prime}, G^{\prime}\right)\right]^{r}$ be the set of pairs $(f, \rho)$ where $f$ is a $C^{r}$-map, and let $\left[X ; X^{\prime}\right]^{r}$ be the space of $C^{r}$-maps from $X$ to $X^{\prime}$. Then when $Y$ is compact, $\left[(Y, G) ;\left(Y^{\prime}, G^{\prime}\right)\right]^{r} / G^{\prime}$ is naturally a Banach orbifold, and since $X=Y / G$ is also compact, $\left[X ; X^{\prime}\right]^{r}$ is a Banach orbifold by Theorem 1.4 of this paper. In this case, (1.5) gives

$$
\left[(Y, G) ;\left(Y^{\prime}, G^{\prime}\right)\right]^{r} / G^{\prime} \subset\left[X ; X^{\prime}\right]^{r}
$$

as an open and closed Banach suborbifold.

The above observation suggests that when developing a theory of orbifolds (based on the theory of maps in this work), one should look at the corresponding theory in the equivariant category first, and then seek a proper generalization to the orbifold category.

An important technique in the equivariant category is given by the various localization theorems. It would be interesting to see if such a technique can be extended to the orbifold category (or more generally the orbispace category). For any orbifold $X$, one can write $X=Y / G$ for a smooth manifold $Y$ and a compact Lie group $G$, where $Y$ is the bundle of orthonormal frames on $X$ and $G=O(n)$ (here $n=\operatorname{dim} X$ ). Technically, one may reduce a "localization problem" for $X$ to a localization problem for $(Y, G)$, for example, as done in the derivation of the index theorem over orbifolds in [22]. However, such an approach is not natural and an intrinsic localization principle would be more desirable.

Not every orbifold can be written as a global quotient $Y / G$. For a general orbifold where $G$ is missing, what would be an appropriate replacement for the "structure group" $G$ ? The answer seems to lie in the notion of "complex of groups" introduced by Haefliger [16]. More concretely, given any orbifold $X$ (or more generally an orbispace), one can associate a complex of groups $G\left(\left\{U_{i}\right\}\right)$ to a given cover $\left\{U_{i}\right\}$ of $X$, where each $U_{i}$ is a uniformized open set, such that for any refinement $\left\{V_{k}\right\}$ of $\left\{U_{i}\right\}$, there is a canonically defined homomorphism from $G\left(\left\{V_{k}\right\}\right)$ to $G\left(\left\{U_{i}\right\}\right)$. In this way, each orbifold is associated with a direct limit of complexes of groups, such that a map between orbifolds induces a "morphism" between the corresponding direct limits of complexes of groups. When $X=Y / G$ is a global quotient and the cover $\left\{U_{i}\right\}$ is taken to be $\{X\}$, the complex of groups $G\left(\left\{U_{i}\right\}\right)$ reduces to the group $G$. See Remark 2.1.2 (7) for more details.

We would like to point out that Haefliger [17] has developed the homological algebra aspects of complexes of groups, generalizing most of the notions having been developed for groups, for instance as in the book of Brown [2].

Summary of main results. We shall develop the theory of maps between orbifolds in the more general context of orbispaces and étale topological groupoids. The following definition of orbispaces is taken from Haefliger [16]. See $§ 2.1$ for a review on étale topological groupoids. 
Definition 1.1 An orbispace is a topological space $X$ equipped with an (equivalence class of ) étale topological groupoid $\Gamma$, such that (1) $X$ is covered by a set of open sets $\left\{U_{i}\right\}$, where for each $U_{i}$ there is a space $\widehat{U_{i}}$ acted on by a discrete group $G_{i}$ such that $U_{i}=\widehat{U}_{i} / G_{i},(2)$ the space of units of $\Gamma$ is the disjoint union $\bigsqcup_{i} \widehat{U}_{i}$ such that the restriction of $\Gamma$ to each $\widehat{U}_{i}$ is the product groupoid $G_{i} \times \widehat{U_{i}}$, and (3) $X$ is the space of $\Gamma$-orbits.

The orbispaces studied in this work are required to further satisfy certain technical conditions, which are given in terms of the defining étale topological groupoid $\Gamma$.

\section{Technical Assumptions:}

(C1) The étale topological groupoid $\Gamma$ is locally connected.

(C2) Denote by $\alpha, \omega$ the maps sending each morphism in $\Gamma$ to its right and left units. For any $U_{i}, U_{j}$, set $\Gamma\left(U_{i}, U_{j}\right)=\left\{\gamma \in \Gamma \mid \alpha(\gamma) \in \widehat{U_{i}}, \omega(\gamma) \in \widehat{U_{j}}\right\}$. Then the restriction of $\alpha$ (resp. $\omega)$ to any connected component of $\Gamma\left(U_{i}, U_{j}\right)$ is a homeomorphism onto a connected component of the image of $\left.\alpha\right|_{\Gamma\left(U_{i}, U_{j}\right)}\left(\right.$ resp. $\left.\left.\omega\right|_{\Gamma\left(U_{i}, U_{j}\right)}\right)$ in $\widehat{U}_{i}\left(\right.$ resp. $\left.\widehat{U}_{j}\right)$.

We remark that the condition (C2) is not preserved under the usual equivalence of étale topological groupoids. For example, consider Thurston's "teardrop" orbifold which is a 2sphere with one orbifold point of order $n$ (cf. [33]). One can easily construct a defining étale topological groupoid $\Gamma$ which does not satisfy $(\mathrm{C} 2)$ (eg. when the space of units of $\Gamma, \bigsqcup_{i} \widehat{U_{i}}$, contains only two connected components). On the other hand, we will show that smooth orbifolds satisfy (C1) and (C2) after taking an appropriate refinement of any given cover of local uniformizing systems (cf. Proposition 2.1.3).

With the preceding understood, the main results in this paper are listed below.

Theorem 1.2 Consider the set of orbispaces which satisfy (C1), (C2). There is a naturally defined notion of maps under which the said set of orbispaces forms a category.

From now on, without explicitly mentioning to the contrary, all orbispaces are assumed to satisfy $(\mathrm{C} 1),(\mathrm{C} 2)$.

The following structural theorem of mapping space will also serve as a technical foundation for the subsequent development. (In this regard, the key technical lemma is Lemma 3.2.2.)

Theorem 1.3 Let $X$ be a paracompact, locally compact and Hausdorff orbispace ${ }^{1}, X^{\prime}$ be any orbispace. The set of maps from $X$ to $X^{\prime}$ is naturally an orbispace (as defined in Definition 1.1) under a canonical étale topological groupoid.

Specializing in the case of smooth orbifolds, one may consider the space of maps of $C^{r}$ class, namely, those maps which can be represented locally by $C^{r}$-maps between local uniformizing systems. A natural topology can be given to the space of $C^{r}$-maps between smooth orbifolds, which generalizes the Whitney topology in the case of smooth manifolds (cf. [19]). The following theorem generalizes the corresponding basic results on smooth manifolds.

Theorem 1.4 Let $X, X^{\prime}$ be any smooth orbifolds where $X$ is compact.

\footnotetext{
${ }^{1}$ see the beginning of $\S 3.2$ for the precise definition of this condition.
} 
(1) The space of $C^{r}$-maps from $X$ to $X^{\prime}$ is naturally a smooth Banach orbifold. In particular, it is Hausdorff and second countable.

(2) For any $l \geq r$, the set of $C^{l}$-maps is a dense subset of the space of $C^{r}$-maps.

Suppose $X, X^{\prime}$ are complete Riemannian orbifolds and $X$ is compact. Then each $C^{r}$-map from $X$ to $X^{\prime}$ has a natural $C^{r}$-norm. (The corresponding topology is called the $C^{r}$-topology.) The following result extends the classical Arzela-Ascoli theorem to the orbifold setting.

Theorem 1.5 Let $X, X^{\prime}$ be any complete Riemannian orbifolds where $X$ is compact. For any sequence of $C^{r}$-maps from $X$ to $X^{\prime}$ which have bounded $C^{r}$-norms, there is a subsequence which converges to a $C^{r-1}$-map in the $C^{r-1}$-topology.

Remark 1.6 (1) A map between orbispaces defined in this paper is a certain equivalence class of homomorphisms between the corresponding defining étale topological groupoids. In this sense, there are several related notions of maps in the literature, cf. Haefliger [14], Hilsum-Skandalis [18], and Pronk [29]. (See also Moerdijk [28]). Our definition is most closely related to that of an Haefliger structure in [14]. More precisely, let $T$ be a locally connected topological space which is trivially regarded as an orbispace, $X$ be an orbispace with a defining étale topological groupoid $\Gamma$. Then a map from $T$ to $X$ defined in this paper may be canonically identified with a $\Gamma$-structure on $T$ defined in [14].

(2) The space of maps as the set of certain equivalence classes of groupoid homomorphisms is naturally the orbit space of a certain "tautological" groupoid. It is not obvious, however, that one can define a nature topology on the groupoid which makes it into an étale topological groupoid. Our main result Theorem 1.3 asserts that this can be done assuming the technical conditions $(\mathrm{C} 1)$ and $(\mathrm{C} 2)$. It would be interesting to know whether these conditions can be removed.

(3) It proved to be important and much more convenient to allow non-effective actions in the local uniformizing systems of an orbifold. This will be assumed throughout the work unless it is explicitly mentioned to the contrary. Theorem 1.4 and Theorem 1.5 remain valid for orbifolds in this more general sense.

(4) The Arzela-Ascoli theorem is fundamental in proofs of various compactness theorems. In [10] the orbifold version of Gromov's compactness theorem for pseudoholomorphic curves was proved by an ad hoc method, relying essentially on the unique continuity property of pseudoholomorphic curves (so were many other arguments in [10]), thus requiring the involved almost complex structures be of $C^{\infty}$ class. Theorem 1.5 allows us to bring the proof of orbifold Gromov Compactness Theorem to the usual line of arguments.

A brief history of this work. Some of the ideas and results in this work were first written down in an article under the title "A homotopy theory of orbispaces" [4] in January 2001, which were also presented in the talk [5] (compare also [25]) in the Madison conference in May 2001. The main point of this preliminary version is that the category of orbispaces introduced in [4] may provide a mathematical framework for considerations originating from the orbifold string theory [12]. More concretely, the following observations were made in [4]. 
(1) Let $X=Y / G$ be an orbifold which is a global quotient. Then the loop space of $X$, ie., the space of maps from $S^{1}$ into $X$, can be identified with the space $P(Y, G) / G$, where

$$
P(Y, G) \equiv\{(\gamma, g) \mid \gamma: \mathbb{R} \rightarrow Y, g \in G \text { such that } \gamma(t+2 \pi)=g \cdot \gamma(t)\}
$$

and $G$ acts on $P(Y, G)$ by $h \cdot(\gamma, g)=(h \circ \gamma, A d(h)(g))$ (cf. Lemma 3.5.1 in [4]).

(2) For any orbifold $X$, the space of "constant" loops in $X$, ie., the fixed-point set of the canonical $S^{1}$-action on the loop space, can be naturally identified with

$$
\widetilde{X} \equiv\left\{\left(p,(g)_{G_{p}}\right) \mid p \in X, g \in G_{p}\right\}
$$

(here $G_{p}$ stands for the isotropy group at $p$ and $(g)_{G_{p}}$ the conjugacy class of $g$ in $G_{p}$ ), which is a disjoint union of orbifolds of various dimensions, containing $X$ as an open and closed suborbifold of top dimension (cf. Proposition 3.5.3 in [4]).

(3) The loop space of an orbifold has a natural infinite dimensional orbifold structure (cf. Theorem 3.5.5 in [4]). (This was also independently observed in [13].)

Based on these observations, we made the following speculations.

(1) Notice that the space $P(Y, G)$ in (1.7) is exactly the configuration space of strings satisfying the so-called "twisted boundary conditions" (cf. (1.1)) considered by the physicists in [12]. Thus observation (1) suggests that the loop space of an orbifold in [4] may serve as the configuration space of strings for string theories of orbifolds in general.

(2) The space $\widetilde{X}$ in (1.8) (first introduced by Kawasaki in [21]) played a key role in the orbifold Gromov-Witten theory [10, 11]. More concretely, the quantum cohomology of an orbifold $X$ is given as an abelian group by the rational cohomology of $\widetilde{X}$ (rather than that of $X$ ) with degrees properly shifted. On the other hand, by the stringy interpretation of quantum cohomology in Vafa [34], the quantum cohomology ring is a deformation of the rational cohomology ring of the space of constant loops. Thus observation (2) suggests that the Gromov-Witten invariants in $[10,11]$ are indeed the "stringy invariants" of orbifolds in [12].

(3) In Witten [35], elliptic genus was interpreted as the $S^{1}$-equivariant index of the Dirac operator on the loop space. By observation (3), one may attempt to develop a theory of orbifold elliptic genera and orbifold elliptic cohomology by extending the constructions in [35] to the loop space of an orbifold. (Later we learned that orbifold elliptic genera had already been studied, cf. [24].) We also remarked in [4] that one may also attempt to extend the work of Chas and Sullivan on "string topology" [3] to the orbifold category, by working with the loop space of orbifold in [4].

The bulk of the current version of this work was carried out in the academic year of 2001-2002, during which time the author was visiting the Institute for Advanced Study in Princeton. The work was completed in its current form in 2003. There were several substantial improvements over the preliminary version [4]. For example, the structural theorems were not available in [4] except for the case of loop space. The homotopy groups defined in [4] were only part of those defined in [6], which are equivalent to the homotopy groups of the corresponding classifying spaces. The CW-complex theory in [6] was not yet developed. 
In January 2001, the author had a conversation with Dennis Sullivan about the subject discussed in [4]. Sullivan pointed out to the author that the existing methods for groupoids or alike work best in the case of cofunctors (eg. cohomology theories). In this regard, the direction taken in the present study is to develop a formalism that works as well in the case of functors.

The rest of this paper is organized as follows.

Section 2 is concerned with a basic foundation for the category of orbispaces introduced in this work. In $\S 2.1$, we present the set of orbispaces which satisfy (C1), (C2) in terms of local charts. This formalism is the most natural one in which $(\mathrm{C} 1),(\mathrm{C} 2)$ are expressed. We end $\S 2.1$ with an elementary proof that smooth orbifolds satisfy (C1), (C2) after taking an appropriate refinement of any given cover of local uniformizing systems. In $\S 2.2$, we discuss the definition of the maps studied in this work, and give the proof of Theorem 1.2.

Section 3 is devoted to a basic structural study on mapping spaces. Theorem 1.3, Theorem 1.4 and Theorem 1.5 are proved in $\S 3.2, \S 3.3$ and $\S 3.4$ respectively. In $\S 3.1$, three preliminary lemmas are presented.

\section{Acknowledgments}

I wish to thank Yongbin Ruan for introducing me to the work of Dixon, Harvey, Vafa and Witten on string theories of orbifolds, and for the joint work on orbifold Gromov-Witten theory, without which the work in this paper and its sequel would not have become possible. I am also very grateful to André Haefliger for several very useful email correspondences, and to Dennis Sullivan for some enlightening conversations. I also wish to thank the Institute for Advanced Study for her warm hospitality and excellent academic atmosphere, where the bulk of this research in its current form was done. This work has been partially supported by NSF grant DMS-9971454, and by the financial support from the Institute for Advanced Study through NSF grant DMS-9729992. During the final preparation, it is partially supported by NSF grant DMS-0304956.

\section{A CATEGORY OF ORBISPACES}

\subsection{Groupoid Versus LOCAL CHART}

We begin by recalling briefly the basic definitions regarding groupoids. See e.g. Haefliger [16] or Bridson-Haefliger [1] for details.

A groupoid $\Gamma$ is a small category whose morphisms are all invertible. The set of objects of $\Gamma$ is naturally identified with the set of units $U$. There are mappings $\alpha, \omega: \Gamma \rightarrow U$ sending each morphism $\gamma \in \Gamma$ to its initial object (also called right unit) and its terminal object (also called left unit) respectively. For any $x \in U$, the set $\Gamma_{x}=\{\gamma \in \Gamma \mid \alpha(\gamma)=\omega(\gamma)=x\}$ is naturally a group, called the isotropy group of $x$. The set $\Gamma \cdot x=\{y \in U \mid \exists \gamma \in \Gamma$ s.t. $\alpha(\gamma)=$ $x, \omega(\gamma)=y\}$ is called the $\Gamma$-orbit of $x$. The set of $\Gamma$-orbits is denoted by $\Gamma \backslash U$.

A topological groupoid is a groupoid $\Gamma$ where $\Gamma$ is also a topological space, such that with the induced topology on the set of units $U$, the mappings $\alpha, \omega: \Gamma \rightarrow U$ as well as the 
mappings of taking composition and inverse are continuous. The set of $\Gamma$-orbits is naturally a topological space with the quotient topology. A topological groupoid is called étale if $\alpha, \omega: \Gamma \rightarrow U$ are local homeomorphisms.

Given any pair $(Y, G)$, where $G$ is a topological group acting continuously on a topological space $Y$ from the left, one may canonically put a topological groupoid structure on $G \times Y$ as follows. Define composition and inverse by $(h, g \cdot y) \circ(g, y)=(h g, y)$ and $(g, y)^{-1}=\left(g^{-1}, g \cdot y\right)$. The space of units is naturally identified with the space $Y$, and $\alpha, \omega$ are given by $\alpha(g, y)=y$ and $\omega(g, y)=g \cdot y$. The groupoid $G \times Y$ is étale if and only if $G$ is a discrete group.

A homomorphism of topological groupoids from $\Gamma$ to $\Gamma^{\prime}$ is a continuous map $\Phi: \Gamma \rightarrow \Gamma^{\prime}$, which commutes with the mappings $\alpha, \omega$ and the mappings of taking composition and inverse, and which induces a continuous map between the spaces of units. When both $\Gamma$ and $\Gamma^{\prime}$ are étale, $\Phi$ is called an equivalence if it is a local homeomorphism and induces a bijection between $\Gamma \backslash U$ and $\Gamma^{\prime} \backslash U^{\prime}$ and an isomorphism $\Gamma_{x} \cong \Gamma_{\Phi(x)}^{\prime}$ for any $x \in U$.

With the preceding understood, we return to the conditions $(\mathrm{C} 1),(\mathrm{C} 2)$ in the introduction. First, by $(\mathrm{C} 1)$ the space of units $U=\bigsqcup_{i} \widehat{U_{i}}$ is locally connected, which implies that the topological space $X=\Gamma \backslash U$ is also locally connected. Second, the étale topological groupoid $\Gamma$ is only determined up to equivalence. Because the condition (C2) may not be preserved under equivalence of étale topological groupoids, it should be understood throughout this work that when an orbispace is said to satisfy (C1), (C2), it is meant that some preferred étale topological groupoid in the equivalence class has been chosen, with respect to which (C1), (C2) are satisfied.

Orbispaces satisfying $(\mathrm{C} 1),(\mathrm{C} 2)$ may be equivalently formulated in a way in terms of local charts instead of groupoids, which is more concrete and geometric. In fact, it is in this formalism that conditions (C1), (C2) are most naturally expressed and sufficiently exploited. We give details of this formulation in the following proposition.

Proposition 2.1.1 Let $X$ be an orbispace under an étale topological groupoid $\Gamma$ which satisfies (C1), (C2). Then $\Gamma$ may be given by the following set of data:

- An atlas of local charts $\left\{\left(\widehat{U}_{i}, G_{U_{i}}, \pi_{U_{i}}\right)\right\}$, where each $U_{i}$ is a connected open subset of $X, \widehat{U_{i}}$ is a locally connected space with continuous left action by a discrete group $G_{U_{i}}$, and $\pi_{U_{i}}: \widehat{U_{i}} \rightarrow X$ is a continuous map inducing a homeomorphism $\widehat{U}_{i} / G_{U_{i}} \cong U_{i}$. Note that the atlas of local charts $\left\{\left(\widehat{U}_{i}, G_{U_{i}}, \pi_{U_{i}}\right)\right\}$ is naturally identified with the set $\mathcal{U}=\left\{U_{i}\right\}$, which forms an open cover of $X$.

- A collection of discrete sets

$$
\mathcal{T}=\left\{T\left(U_{i}, U_{j}\right) \mid U_{i}, U_{j} \in \mathcal{U} \text {, s.t. } U_{i} \cap U_{j} \neq \emptyset\right\}
$$

which satisfies the following conditions:

$-T\left(U_{i}, U_{i}\right)=G_{U_{i}}$, and for any $i \neq j, T\left(U_{i}, U_{j}\right)=\bigsqcup_{u \in I_{i j}} T_{W_{u}}\left(U_{i}, U_{j}\right)$ where $\left\{W_{u} \mid\right.$ $\left.u \in I_{i j}\right\}$ is the set of connected components of $U_{i} \cap U_{j}$.

- Each $\xi \in T_{W_{u}}\left(U_{i}, U_{j}\right)$ is assigned with a homeomorphism $\phi_{\xi}$, whose domain and range are connected components of the inverse image of $W_{u}$ in $\widehat{U}_{i}$ and $\widehat{U}_{j}$ respectively, such that $\pi_{U_{i}}=\pi_{U_{j}} \circ \phi_{\xi}$. (For each $\xi \in G_{U_{i}}$, we define $\phi_{\xi}$ to be the self-homeomorphism of $\widehat{U_{i}}$ induced by the action of $\xi$.) 
- For any $\xi \in T\left(U_{i}, U_{j}\right), \eta \in T\left(U_{j}, U_{k}\right)$, and any $x \in$ Domain $\left(\phi_{\xi}\right)$ such that $\phi_{\xi}(x) \in \operatorname{Domain}\left(\phi_{\eta}\right)$, there exists an $\eta \circ \xi(x) \in T\left(U_{i}, U_{k}\right)$ such that $\phi_{\eta \circ \xi(x)}(x)=$ $\phi_{\eta}\left(\phi_{\xi}(x)\right)$. Moreover, $x \mapsto \eta \circ \xi(x)$ is locally constant, and the composition $(\xi, \eta) \mapsto \eta \circ \xi(x)$ is associative and coincides with the group multiplication in $G_{U_{i}}$ when restricted to $T\left(U_{i}, U_{i}\right)=G_{U_{i}}$.

- Every $\xi \in T\left(U_{i}, U_{j}\right)$ has an inverse $\xi^{-1} \in T\left(U_{j}, U_{i}\right)$, such that Domain $\left(\phi_{\xi}\right)=$ Range $\left(\phi_{\xi^{-1}}\right)$, Domain $\left(\phi_{\xi^{-1}}\right)=$ Range $\left(\phi_{\xi}\right)$, and $\xi^{-1} \circ \xi(x)=1 \forall x \in$ Domain $\left(\phi_{\xi}\right)$, $\xi \circ \xi^{-1}(x)=1 \forall x \in \operatorname{Domain}\left(\phi_{\xi^{-1}}\right)$.

Proof Assume that $\Gamma$ is given, satisfying $(\mathrm{C} 1),(\mathrm{C} 2)$, and that the space of units of $\Gamma$ is the disjoint union $\bigsqcup_{i} \widehat{U}_{i}$ with the restriction to each $\widehat{U}_{i}$ being the product groupoid $G_{i} \times \widehat{U}_{i}$. We obtain the atlas of local charts by setting $G_{U_{i}}=G_{i}$, and $\pi_{U_{i}}: \widehat{U}_{i} \rightarrow X$ the orbit map. (Without loss of generality, we may always assume that $U_{i}=\widehat{U}_{i} / G_{i}$ is connected.)

As for the collection of discrete sets $\mathcal{T}=\left\{T\left(U_{i}, U_{j}\right)\right\}$, we define $T\left(U_{i}, U_{i}\right)=G_{U_{i}}$, and for any $i \neq j$, we define $T\left(U_{i}, U_{j}\right)$ to be the set of connected components of $\Gamma\left(U_{i}, U_{j}\right)=\{\gamma \in$ $\left.\Gamma \mid \alpha(\gamma) \in \widehat{U_{i}}, \omega(\gamma) \in \widehat{U_{j}}\right\}$. For any $\xi \in T\left(U_{i}, U_{j}\right), i \neq j$, by $(\mathrm{C} 2)$, there exists an inverse of $\alpha$ defined from a connected component of the image of $\left.\alpha\right|_{\Gamma\left(U_{i}, U_{j}\right)}$ in $\widehat{U}_{i}$ to the connected component of $\Gamma\left(U_{i}, U_{j}\right)$ which is named by $\xi$. We define $\phi_{\xi}=\omega \circ s_{\xi}$ where $s_{\xi}$ is the said inverse of $\alpha$. We define $\eta \circ \xi(x)$ to be the connected component which contains the morphism $s_{\eta}\left(\phi_{\xi}(x)\right) \circ s_{\xi}(x)$, and define the inverse $\xi^{-1}$ to be the connected component which contains the morphism $s_{\xi}(x)^{-1}$ for some (and hence for all) $x \in$ Domain $\left(\phi_{\xi}\right)$. It is easy to verify that these objects satisfy the claimed conditions. We leave the details to the reader.

Conversely, assume that the said set of data is given, we may recover the groupoid $\Gamma$ as follows. Define $\Gamma$ to be the subset of $\bigsqcup_{i, j} T\left(U_{i}, U_{j}\right) \times \widehat{U_{i}}$ with induced topology which consists

of pairs $(\xi, x)$ such that $x \in$ Domain $\left(\phi_{\xi}\right)$. The space of units is the disjoint union $\bigsqcup_{i} \widehat{U}_{i}$ via the identification $x \mapsto(1, x)$. The groupoid structure of $\Gamma$ is given as follows: $\alpha(\xi, x)=x$, $\omega(\xi, x)=\phi_{\xi}(x),(\eta, y) \circ(\xi, x)=(\eta \circ \xi(x), x)$ where $y=\phi_{\xi}(x)$, and $(\xi, x)^{-1}=\left(\xi^{-1}, \phi_{\xi}(x)\right)$. It is an easy exercise to verify that $\Gamma$ is an étale topological groupoid which satisfies (C1), (C2). We leave the details to the reader.

Some remarks are in order to further explain a few points.

\section{Remark 2.1.2}

(1) One may always require that each local chart $\widehat{U_{i}}$ is also connected by replacing it with one of its connected component, which amounts to replace $\Gamma$ with an equivalent groupoid such that $(\mathrm{C} 1),(\mathrm{C} 2)$ are still satisfied.

(2) Since $x \mapsto \eta \circ \xi(x)$ is locally constant, $\eta \circ \xi(x)$ depends only on the connected component of $\phi_{\xi}^{-1}$ (Domain $\left.\left(\phi_{\eta}\right)\right)$ in which $x$ lies. More generally, for any sequence $\xi_{k, k+1} \in T\left(U_{k}, U_{k+1}\right)$, $1 \leq k \leq n-1$, such that

$$
\phi_{\xi_{n-1, n}} \circ \cdots \circ \phi_{\xi_{23}} \circ \phi_{\xi_{12}}
$$

is defined, then $\xi_{n-1, n} \circ \cdots \circ \xi_{23} \circ \xi_{12}(x)$ depends only on the connected component of the domain of (2.1.1) in which $x$ lies. We introduce

(2.1.2) $\Lambda\left(\xi_{12}, \xi_{23}, \cdots, \xi_{n-1, n}\right)=\{\mathbf{a} \mid \mathbf{a}$ is a connected component of the domain of (2.1.1)\}, 
and for any $\mathbf{a} \in \Lambda\left(\xi_{12}, \xi_{23}, \cdots, \xi_{n-1, n}\right)$, set

$$
\xi_{n-1, n} \circ \cdots \circ \xi_{23} \circ \xi_{12}(\mathbf{a})=\xi_{n-1, n} \circ \cdots \circ \xi_{23} \circ \xi_{12}(x), \forall x \in \mathbf{a} .
$$

When there is no ambiguity, we simply write $\xi_{n-1, n} \circ \cdots \circ \xi_{23} \circ \xi_{12}$ for $\xi_{n-1, n} \circ \cdots \circ \xi_{23} \circ \xi_{12}(\mathbf{a})$.

(3) For any $\xi \in T\left(U_{i}, U_{j}\right)$, there exists an isomorphism of groups $\lambda_{\xi}$ defined by

$$
\lambda_{\xi}(g)=\xi \circ g \circ \xi^{-1}(x),
$$

where $x$ is any point in Domain $\left(\phi_{\xi^{-1}}\right)$ and $g$ is in the subgroup of $G_{U_{i}}$ fixing the set Domain $\left(\phi_{\xi}\right)$. The range of $\lambda_{\xi}$ is the subgroup of $G_{U_{j}}$ fixing the set Range $\left(\phi_{\xi}\right)$. In particular, for any $\xi \in T\left(U_{i}, U_{i}\right)=G_{U_{i}}, \lambda_{\xi}=A d(\xi): G_{U_{i}} \rightarrow G_{U_{i}}$. We observe that for any $\xi, \phi_{\xi}$ is $\lambda_{\xi}$-equivariant, and the pairs $\left(\phi_{\xi}, \lambda_{\xi}\right)$ satisfy

$$
\left(\phi_{\eta \circ \xi(\mathbf{a})}, \lambda_{\eta \circ \xi(\mathbf{a})}\right)=\left(\phi_{\eta}, \lambda_{\eta}\right) \circ\left(\phi_{\xi}, \lambda_{\xi}\right)
$$

when restricted to $\left(\mathbf{a}, G_{\mathbf{a}}\right)$ for any $\mathbf{a} \in \Lambda(\xi, \eta)$, where $G_{\mathbf{a}}$ is the subgroup of Domain $\left(\lambda_{\xi}\right)$ which fixes a. Hence each element $\xi$ is associated with a 'transformation of local charts' $\left(\phi_{\xi}, \lambda_{\xi}\right)$. Roughly speaking, (C2) dictates that each 'transformation of local charts' be defined over a domain that is maximally large as possible. In a certain sense, étale topological groupoids satisfying (C1), (C2) are the closest generalization of the product groupoids, or in other words, orbispaces satisfying $(\mathrm{C} 1),(\mathrm{C} 2)$ are the closest generalization of the orbispaces of global quotients.

(4) We may maximize the atlas of local charts by adding all the connected open subsets of each $U_{i} \in \mathcal{U}$ to $\mathcal{U}=\left\{U_{i}\right\}$. This amounts to change $\Gamma$ to an equivalent groupoid (still satisfying (C1), (C2)), which is done as follows. Let $V$ be any connected open subset of some $U_{i_{0}} \in \mathcal{U}$, and $\widehat{V}$ be the inverse image of $V$ in $\widehat{U_{i_{0}}}$. We define a groupoid $\Gamma_{1}=\left.\Gamma \sqcup \Gamma\right|_{\widehat{V}} \sqcup \Gamma^{\prime}$ where $\Gamma^{\prime}=\{\gamma \in \Gamma \mid$ either $\alpha(\gamma) \in \widehat{V}$ or $\omega(\gamma) \in \widehat{V}\}$. The space of units of $\Gamma_{1}$ is the disjoint union $\widehat{V} \sqcup\left(\bigsqcup_{i} \widehat{U}_{i}\right)$, hence the atlas of local charts for $\Gamma_{1}$ is $\{V\} \cup \mathcal{U}$. Moreover, the inclusion $\Gamma \subset \Gamma_{1}$ induces an equivalence of groupoids, and $\Gamma_{1}$ satisfies (C1), (C2). One may iterate this process to maximize $\mathcal{U}$ by Zorn's Lemma.

(5) We introduce the following equivalence relation: two orbispace structures on $X$ is said to be directly equivalent if the atlas of local charts of one of them is contained in that of the other, and is said to be equivalent if they are related by a finite chain of directly equivalent orbispace structures.

(6) Product groupoids $G \times Y$, where $Y$ is locally connected, satisfy (C1), (C2) trivially. Thus the global quotient spaces are the most basic examples of orbispaces considered in this work. Other known examples of orbispaces satisfying (C1), (C2) include smooth orbifolds (which is shown next), and orbihedra of Haefliger in [16]. On the other hand, this class of orbispaces is closed under taking subspaces in the following sense. Let $Y$ be a subset of $X$ such that the inverse image of $Y$ in $\bigsqcup_{i} \widehat{U_{i}}, \widehat{Y}$, is locally connected. Then $Y$ becomes an orbispace under the groupoid $\left.\Gamma\right|_{\widehat{Y}}$, which also satisfies $(\mathrm{C} 1),(\mathrm{C} 2)$.

(7) We shall explain here that, with the technical assumptions (C1) and (C2), each orbispace may be associated with a direct limit of complexes of groups defined in Haefliger [16]. We hope that this direct limit would play the role of 'structure group' (ie. the role of $G$ in a global quotient $X=Y / G$ ) for a general orbispace. 
We first recall the definition of complex of groups. Let $K$ be a simplicial cell complex. We set $V(K)$ for the set of barycenters of cells of $K$, and $E(K)$ for the set of edges of the barycentric subdivision of $K$. Each edge $a \in E(K)$ is natually oriented, ie., if the initial point $i(a)$ of $a$ is the barycenter of a cell $\sigma$ and the terminal point $t(a)$ is the barycenter of $\tau$, then $\operatorname{dim} \tau<\operatorname{dim} \sigma$. Two edges $a, b \in E(K)$ are said to be composable if $i(a)=t(b)$ and the composition $c=a b$ is the edge with $i(c)=i(b)$ and $t(c)=t(a)$ such that $a, b$ and $c$ form the boundary of a 2-simplex in the barycentric subdivision of $K$.

With the above notations understood, a complex of groups $G(K)=\left(K, G_{\sigma}, \psi_{a}, g_{a, b}\right)$ on $K$ is given by the following data:

(1) a group $G_{\sigma}$ for each cell $\sigma \in V(K)$,

(2) an injective homomorphism $\psi_{a}: G_{i(a)} \rightarrow G_{t(a)}$ for each edge $a \in E(K)$,

(3) an element $g_{a, b} \in G_{t(a)}$ for each pair $a, b \in E(K)$ of composable edges such that

$$
A d\left(g_{a, b}\right) \circ \psi_{a b}=\psi_{a} \circ \psi_{b},
$$

and the set of elements $\left\{g_{a, b}\right\}$ satisfies the cocycle condition

$$
\psi_{a}\left(g_{b, c}\right) g_{a, b c}=g_{a, b} g_{a b, c}
$$

for any triple $a, b, c \in E(K)$ of composable edges.

Homomorphisms of complexes of groups are defined as follows. Let $G(K)=\left(K, G_{\sigma}, \psi_{a}, g_{a, b}\right)$, $G\left(K^{\prime}\right)=\left(K^{\prime}, G_{\sigma^{\prime}}^{\prime}, \psi_{a^{\prime}}^{\prime}, g_{a^{\prime}, b^{\prime}}^{\prime}\right)$ be complexes of groups on $K, K^{\prime}$ respectively, and let $f: K \rightarrow$ $K^{\prime}$ be a simplicial map. A homomorphism $\phi=\left(f, \phi_{\sigma}, g_{a}^{\prime}\right): G(K) \rightarrow G\left(K^{\prime}\right)$ over $f$ is given by the following data:

(1) a homomorphism $\phi_{\sigma}: G_{\sigma} \rightarrow G_{f(\sigma)}^{\prime}$ for each cell $\sigma \in V(K)$,

(2) an element $g_{a}^{\prime} \in G_{f(t(a))}^{\prime}$ for each edge $a \in E(K)$ such that

$$
A d\left(g_{a}^{\prime}\right) \circ \psi_{f(a)}^{\prime} \circ \phi_{i(a)}=\phi_{t(a)} \circ \psi_{a},
$$

and for each pair $a, b \in E(K)$ of composable edges,

$$
\phi_{t(a)}\left(g_{a, b}\right) g_{a b}^{\prime}=g_{a}^{\prime} \psi_{f(a)}^{\prime}\left(g_{b}^{\prime}\right) g_{f(a), f(b)}^{\prime} .
$$

(Note: when $f(a)$ is not a well-defined edge in $E\left(K^{\prime}\right)$, ie. when $f(i(a))=f(t(a))$, we set $\psi_{f(a)}^{\prime}=i d: G_{f(i(a))}^{\prime} \rightarrow G_{f(t(a))}^{\prime}$, and moreover, we set $g_{f(a), f(b)}^{\prime}=1$ if either $f(a)$ or $f(b)$ is not well-defined.)

Finally, two complexes of groups $\left(K, G_{\sigma}, \psi_{a}, g_{a, b}\right),\left(K, G_{\sigma}, \psi_{a}^{\prime}, g_{a, b}^{\prime}\right)$ on $K$ are said to differ by a coboundary if there is a homomorphism $\phi=\left(i d, i d, g_{a}\right)$ over $i d: K \rightarrow K$ between them.

Now let $X$ be an orbispace with atlas of local charts $\mathcal{U}$. For any cover $\left\{U_{i}\right\}$ of $X$ where $U_{i} \in \mathcal{U}$, we define a complex of groups $G\left(\left\{U_{i}\right\}\right)$ as follows. The corresponding simplicial cell complex $K\left(\left\{U_{i}\right\}\right)$ consists of $n$-cells $\left(\left\{U_{i_{0}}, \cdots, U_{i_{n}}\right\}, j\right), n \geq 0$, where $U_{i_{0}}, \cdots, U_{i_{n}}$ are distinct elements in $\left\{U_{i}\right\}$ such that $U_{i_{0}} \cap \cdots \cap U_{i_{n}} \neq \emptyset$, and $j$ is a connected component of $U_{i_{0}} \cap \cdots \cap U_{i_{n}}$. The faces of $\left(\left\{U_{i_{0}}, \cdots, U_{i_{n}}\right\}, j\right)$ are obtained by removing one of $U_{i_{k}}$ 's and by replacing $j$ with the corresponding connected component containing $j$.

To obtain the remaining data, we rely on the following fact due to the assumptions $(\mathrm{C} 1)$ and (C2): For each cell $\sigma=\left(\left\{U_{i_{0}}, \cdots, U_{i_{n}}\right\}, j\right), j$ is a local chart of $X$, which we denote by $U_{\sigma}$. With this understood, we define the group $G_{\sigma}$ in $G\left(\left\{U_{i}\right\}\right)$ associated to $\sigma$ by $G_{\sigma} \equiv G_{U_{\sigma}}$. Now observe that an edge $a$ with $i(a)=\sigma$ corresponds to removing one or several $U_{i_{k}}$ 's in 
$\left\{U_{i_{0}}, \cdots, U_{i_{n}}\right\}$, and if we let $j_{a}$ be the corresponding connected component for the cell $t(a)$, we have $j \subset j_{a}$. We define $\psi_{a}$ in $G\left(\left\{U_{i}\right\}\right)$ by fixing a $\xi \in T\left(j, j_{a}\right)$ and set $\psi_{a} \equiv \lambda_{\xi}$ (see (2.1.3) for the definition of $\lambda_{\xi}$ ). Then it follows easily that there are unique elements $g_{a, b}$ such that (2.1.4a) and (2.1.4b) are satisfied, thus giving the construction of $G\left(\left\{U_{i}\right\}\right)$. If we choose a different $\xi \in T\left(j, j_{a}\right)$, the corresponding complex of groups differs by a coboundary.

We leave to the reader to verify that for any refinement $\left\{V_{k}\right\}$ of $\left\{U_{i}\right\}$, there is a canonically defined homomorphism from $G\left(\left\{V_{k}\right\}\right)$ to $G\left(\left\{U_{i}\right\}\right)$, so that in this way, the orbispace $X$ is associated with a direct limit of complexes of groups. Moreover, a map between two orbispaces (to be defined in the next subsection) induces a "morphism" between the corresponding direct limits of complexes of groups. Finally, we observe that if $X=Y / G$ and $X$ is connected, and $\left\{U_{i}\right\}=\{X\}$, then the simplicial cell complex $K\left(\left\{U_{i}\right\}\right)$ consists of a single vertex, and the complex of groups $G\left(\left\{U_{i}\right\}\right)$ reduces to the group $G$.

The remainder of this subsection is occupied with an elementary proof that after taking a suitable refinement of any given cover of local uniformizing systems, smooth orbifolds satisfy (C1), (C2). We remark that passing to a refinement is necessary here, as one can easily construct an example not satisfying (C2) using Thurston's 'teardrop' orbifold (cf. [33]).

Recall the definition of orbifolds due to Satake [31]. Suppose $X$ is a Hausdorff, paracompact space. An orbifold structure on $X$ is given by an open cover $\left\{U_{i}\right\}$ satisfying the following conditions: (1) Each $U_{i}$ is given with a uniformizing system $\left(\widehat{U_{i}}, G_{i}, \pi_{i}\right)$, where $\widehat{U_{i}}$ is a connected open subset of $\mathbb{R}^{n}, G_{i}$ is a finite group of self-diffeomorphisms of $\widehat{U}_{i}$, and $\pi_{i}: \widehat{U}_{i} \rightarrow U_{i}$ is a continuous map inducing a homeomorphism $\widehat{U}_{i} / G_{i} \cong U_{i}$. (2) For any pair $U_{i}, U_{j}$ such that $U_{j} \subset U_{i}$, there is an associated set of injections $\{\phi\}$, where $\phi: \widehat{U_{j}} \rightarrow \widehat{U_{i}}$ is an open embedding such that $\pi_{i} \circ \phi=\pi_{j}$. The group $G_{i}$ acts on the set of injections freely and transitively via post-compositions. Moreover, for any $U_{k} \subset U_{j} \subset U_{i}$, the composition of an injection associated to $U_{k} \subset U_{j}$ with an injection associated to $U_{j} \subset U_{i}$ is an injection associated to $U_{k} \subset U_{i}$. (3) For any point $p \in X$ such that $p \in U_{i} \cap U_{j}$, there is a $U_{k}$ satisfying $p \in U_{k}$ and $U_{k} \subset U_{i} \cap U_{j}$. In Satake's original definition, it was assumed that for each $i$ the fixed-point set of any element of $G_{i}$ in $\widehat{U}_{i}$ is of codimension at least 2. This assumption implies the properties about injections listed in Condition (2) above. Later in [21], Kawasaki removed Satake's original assumption, and instead, he imposed these properties as part of the axioms in the definition of orbifolds.

The orbifolds of Satake may also be described equivalently using étale topological groupoids. More concretely, let $U=\bigsqcup_{i} \widehat{U}_{i}$ be the disjoint union. Then the set of open embeddings $\{\phi\}$ generates a pseudogroup of local diffeomorphisms of $U$. The associated étale topological groupoid $\Gamma$, which is the space of germs given with the étale topology, defines an orbispace structure on $X$ in the sense of Definition 1.1. See e.g. [1] for more details.

Proposition 2.1.3 Given any orbifold $\left(X,\left\{U_{i}\right\}\right)$ as defined by Satake, with the associated étale topological groupoid denoted by $\Gamma$, there is a refinement $\left\{V_{\alpha}\right\}$ of $\left\{U_{i}\right\}$, such that the restriction of $\Gamma$ to the inverse image of $\bigsqcup_{\alpha} V_{\alpha}$ in $\bigsqcup_{i} \widehat{U_{i}}$ is an equivalent groupoid with respect to which (C1), (C2) are satisfied. 
Proof We need to assume an auxiliary Riemannian metric on the orbifold $X$. Recall that a Riemannian metric on $X$ is a collection of metrics $\left\{g_{i}\right\}$ where $g_{i}$ is a $G_{i}$-equivariant Riemannian metric on $\widehat{U}_{i}$ with respect to which each injection is isometric. Such a metric on $X$ exists because $X$ is paracompact. Given a Riemannian metric on $X$, a path $\gamma: I \rightarrow X$ is called a geodesic if for any $t \in I$, the restriction of $\gamma$ in a neighborhood of $t$ can be lifted to a geodesic $\tilde{\gamma}_{t}$ in $\widehat{U}_{i}$ for some $i$. We observe two facts about geodesics: (1) the length of a geodesic $\gamma: I \rightarrow X$ is well-defined, which is $\ell(\gamma)=\int_{I}\left|\frac{d \tilde{\gamma}_{t}}{d t}(t)\right| d t$, and (2) if $\gamma(I) \subset U_{i}$ for some $i$, then there is a geodesic $\tilde{\gamma}: I \rightarrow \widehat{U}_{i}$ such that $\gamma=\pi_{i} \circ \tilde{\gamma}$, and moreover, $\ell(\gamma)=\ell(\tilde{\gamma})=\int_{I}\left|\frac{d \tilde{\gamma}}{d t}\right| d t$.

Given any point $p \in X$, suppose $p \in U_{i}$ for some $i$ and let $\hat{p} \in \pi_{i}^{-1}(p) \subset \widehat{U}_{i}$. There is a family of neighborhoods $U_{p}(r)$ of $p$ in $X$, parameterized by $r$ where $0<r \leq r_{p}$ for some $r_{p}$ depending only on $p$ and the fixed Riemannian metric. Each $U_{p}(r)$ has a natural uniformizing system $\left(B_{p}(r), G_{p}, \pi_{p}\right)$, where $B_{p}(r)$ is the open ball of radius $r$ in $\left(T \widehat{U}_{i}\right)_{\hat{p}}$ centered at the origin, $G_{p}$ is the stabilizer of $G_{i}$ at $\hat{p}$, and $\pi_{p}:\left(T \widehat{U}_{i}\right)_{\hat{p}} \rightarrow X$ is the map sending each line $t v$, where $v \in\left(T \widehat{U}_{i}\right)_{\hat{p}}$, to a geodesic $\gamma_{v}$ in $X$ such that $\gamma_{v}(0)=p$ and locally at $0, \gamma_{v}$ can be lifted to a geodesic $\tilde{\gamma}_{v}$ in $\widehat{U}_{i}$ with $\tilde{\gamma}_{v}(0)=\hat{p}$ and $\frac{d \tilde{\gamma}_{v}}{d t}(0)=v$. These neighborhoods $U_{p}(r)$ have the following nice property: for any $U_{i} \in\left\{U_{i}\right\}$, if $U_{p}(r) \subset U_{i}$, then the uniformizing system $\left(B_{p}(r), G_{p}, \pi_{p}\right)$ is isomorphic to an induced one of $U_{p}(r)$ from $\left(\widehat{U}_{i}, G_{i}, \pi_{i}\right)$. Each $B_{p}(r)$ inherits a $G_{p}$-equivariant metric from the auxiliary metric on $X$. We may further assume that all $\overline{B_{p}(r)}, r \leq r_{p}$, are geodesically convex, meaning that any two points in $\overline{B_{p}(r)}$ are joined by a unique geodesic in $\overline{B_{p}(r)}$ such that the distance from a point on the geodesic to the center of $\overline{B_{p}(r)}$ is a strict convex function.

Let $\mathcal{W}=\left\{U_{p}(r) \mid p \in X, r \leq \frac{r_{p}}{5}\right\}$. We will show next that for any $U_{p_{1}}\left(r_{1}\right), \cdots, U_{p_{n}}\left(r_{n}\right) \in \mathcal{W}$ with $r_{p_{1}} \leq r_{p_{k}}, 2 \leq k \leq n$, and $U_{p_{1}}\left(r_{1}\right) \cap \cdots \cap U_{p_{n}}\left(r_{n}\right) \neq \emptyset$, the subset $\pi_{p_{1}}^{-1}\left(\overline{U_{p_{1}}\left(r_{1}\right)} \cap \cdots \cap\right.$ $\left.\overline{U_{p_{n}}\left(r_{n}\right)}\right)$ of $\overline{B_{p_{1}}\left(r_{1}\right)}$ has the property that every two points in $\pi_{p_{1}}^{-1}\left(\overline{U_{p_{1}}\left(r_{1}\right)} \cap \cdots \cap \overline{U_{p_{n}}\left(r_{n}\right)}\right)$ are joined by a unique geodesic in $\pi_{p_{1}}^{-1}\left(\overline{U_{p_{1}}\left(r_{1}\right)} \cap \cdots \cap \overline{U_{p_{n}}\left(r_{n}\right)}\right)$. The proof goes as follows. Suppose $x_{1}, x_{2}$ are any two points in $\pi_{p_{1}}^{-1}\left(\overline{U_{p_{1}}\left(r_{1}\right)} \cap \cdots \cap \overline{U_{p_{n}}\left(r_{n}\right)}\right)$. Let $\tilde{\gamma}_{1}$ be the unique geodesic in $\overline{B_{p_{1}}\left(r_{1}\right)}$ connecting $x_{1}, x_{2}$. We shall prove that $\tilde{\gamma}_{1}$ lies in the subset $\pi_{p_{1}}^{-1}\left(\overline{U_{p_{1}}\left(r_{1}\right)} \cap \cdots \cap \overline{U_{p_{n}}\left(r_{n}\right)}\right)$. It suffices to show that $\gamma=\pi_{p_{1}}\left(\tilde{\gamma}_{1}\right)$ lies in $\overline{U_{p_{k}}\left(r_{k}\right)}$ for any $2 \leq k \leq n$. To this end, recall that we have assumed that $r_{p_{1}} \leq r_{p_{k}}$ for any $2 \leq k \leq n$. It follows that $\ell(\gamma) \leq 2 r_{1} \leq \frac{2 r_{p_{1}}}{5} \leq \frac{2 r_{p_{k}}}{5}$, so that the distance between $p_{k}$ and any point on $\gamma$ is less than or equal to $r_{k}+\frac{2 r_{p_{k}}}{5} \leq \frac{3 r_{p_{k}}}{5}$, which implies that $\gamma$ lies in $U_{p_{k}}\left(r_{p_{k}}\right)$. Hence there is a geodesic lifting $\tilde{\gamma}_{k}$ of $\gamma$ into $B_{p_{k}}\left(r_{p_{k}}\right)$, with the end points of $\tilde{\gamma}_{k}$ lying in $\overline{B_{p_{k}}\left(r_{k}\right)}$. Now recall that all $\overline{B_{p_{k}}(r)}, r \leq r_{p_{k}}$, are geodesically convex. Hence $\tilde{\gamma}_{k}$ must lie in $\overline{B_{p_{k}}\left(r_{k}\right)}$, and therefore $\gamma=\pi_{p_{k}}\left(\tilde{\gamma}_{k}\right)$ must lie in $\overline{U_{p_{k}}\left(r_{k}\right)}$.

Note that each $\pi_{p_{1}}^{-1}\left(U_{p_{1}}\left(r_{1}\right) \cap \cdots \cap U_{p_{n}}\left(r_{n}\right)\right)$ is a convex, topological ball. In particular, $U_{p_{1}}\left(r_{1}\right) \cap \cdots \cap U_{p_{n}}\left(r_{n}\right)$ is connected.

Now we take a refinement of $\left\{U_{i}\right\}$, denoted by $\mathcal{V}=\left\{V_{\alpha}\right\}$, where each $V_{\alpha} \in \mathcal{W}$. Let $V=\bigsqcup_{U_{p}(r) \in \mathcal{V}} B_{p}(r)$ be the disjoint union. Then the étale topological groupoid $\Gamma$ induces an equivalent étale topological groupoid $\Gamma^{\prime}$ having $V$ as the space of units. ( $\Gamma^{\prime}$ is simply the restriction of $\Gamma$ by thinking $V$ as a subset of $\bigsqcup_{i} \widehat{U}_{i}$, the space of units of $\Gamma$.) We shall prove that $\Gamma^{\prime}$ satisfies $(\mathrm{C} 1)$, (C2). 
First, $(\mathrm{C} 1)$ is trivial. To verify $(\mathrm{C} 2)$, assume $U_{p_{1}}\left(r_{1}\right), U_{p_{2}}\left(r_{2}\right) \in \mathcal{V}$ have nonempty intersection, and without loss of generality, assume $r_{p_{1}} \leq r_{p_{2}}$. Set $\Gamma\left(U_{p_{1}}\left(r_{1}\right), U_{p_{2}}\left(r_{2}\right)\right)=\{\gamma \in$ $\left.\Gamma^{\prime} \mid \alpha(\gamma) \in B_{p_{1}}\left(r_{1}\right), \omega(\gamma) \in B_{p_{2}}\left(r_{2}\right)\right\}$. Then the image of $\left.\alpha\right|_{\Gamma\left(U_{p_{1}}\left(r_{1}\right), U_{p_{2}}\left(r_{2}\right)\right)}$ in $B_{p_{1}}\left(r_{1}\right)$ is $\pi_{p_{1}}^{-1}\left(U_{p_{1}}\left(r_{1}\right) \cap U_{p_{2}}\left(r_{2}\right)\right)$, which is a convex ball by the nature of the set $\mathcal{W}$. Since $\left.\alpha\right|_{\Gamma\left(U_{p_{1}}\left(r_{1}\right), U_{p_{2}}\left(r_{2}\right)\right)}$ is a covering map, it must be a homeomorphism onto its image when restricted to each connected component of $\Gamma\left(U_{p_{1}}\left(r_{1}\right), U_{p_{2}}\left(r_{2}\right)\right)$. In particular, each connected component of $\Gamma\left(U_{p_{1}}\left(r_{1}\right), U_{p_{2}}\left(r_{2}\right)\right)$ is homeomorphic to an open ball. On the other hand, $\left.\omega\right|_{\Gamma\left(U_{p_{1}}\left(r_{1}\right), U_{p_{2}}\left(r_{2}\right)\right)}$ is a covering map onto $\pi_{p_{2}}^{-1}\left(U_{p_{1}}\left(r_{1}\right) \cap U_{p_{2}}\left(r_{2}\right)\right)$. The group of deck transformations for any connected component is isomorphic to a subgroup $K$ of $H$, where $H$ is the subgroup of $G_{p_{1}}$ which leaves the subset $\pi_{p_{1}}^{-1}\left(U_{p_{1}}\left(r_{1}\right) \cap U_{p_{2}}\left(r_{2}\right)\right)$ of $B_{p_{1}}\left(r_{1}\right)$ invariant. Furthermore, $K$ acts on $\pi_{p_{1}}^{-1}\left(U_{p_{1}}\left(r_{1}\right) \cap U_{p_{2}}\left(r_{2}\right)\right)$ freely. But this is impossible unless $K$ is trivial, because the action of $K$ is easily seen to extend over to the closure of $\pi_{p_{1}}^{-1}\left(U_{p_{1}}\left(r_{1}\right) \cap U_{p_{2}}\left(r_{2}\right)\right.$ ), which is a closed ball. By Brouwer's fixed point theorem, it can not be free unless $K$ is trivial. Thus $\left.\omega\right|_{\Gamma\left(U_{p_{1}}\left(r_{1}\right), U_{p_{2}}\left(r_{2}\right)\right)}$ must be a homeomorphism onto its image also when restricted to each connected component of $\Gamma\left(U_{p_{1}}\left(r_{1}\right), U_{p_{2}}\left(r_{2}\right)\right)$. This verifies $(\mathrm{C} 2)$.

\subsection{EquiVAlenCe Class of GROUPOID HOMOMORPHISMS}

Let $X$ be an orbispace, defined by an étale topological groupoid $\Gamma$. Denote by $\mathcal{U}=\left\{U_{i}\right\}$ the atlas of local charts on $X$. Then any subset $\left\{U_{\alpha}\right\}$ of $\mathcal{U}$ is associated with an étale topological groupoid $\Gamma\left\{U_{\alpha}\right\}$, which is the restriction of $\Gamma$ to $\bigsqcup_{\alpha} \widehat{U_{\alpha}}$. More explicitly, $\Gamma\left\{U_{\alpha}\right\}$ is the subset of $\bigsqcup_{\alpha, \beta} T\left(U_{\alpha}, U_{\beta}\right) \times \widehat{U_{\alpha}}$ which consists of pairs $(\xi, x)$ such that $x \in \operatorname{Domain}\left(\phi_{\xi}\right)$.

Lemma 2.2.1 Let $X, X^{\prime}$ be orbispaces whose atlas of local charts are denoted by $\mathcal{U}=$ $\left\{U_{i}\right\}, \mathcal{U}^{\prime}=\left\{U_{i^{\prime}}^{\prime}\right\}$ respectively, and $\left\{U_{\alpha}\right\} \subset \mathcal{U},\left\{U_{\alpha^{\prime}}^{\prime}\right\} \subset \mathcal{U}^{\prime}$ be any subsets where each $\widehat{U_{\alpha}}$ is connected. Then any homomorphism from $\Gamma\left\{U_{\alpha}\right\}$ to $\Gamma\left\{U_{\alpha^{\prime}}^{\prime}\right\}$ may be written as $\left(\left\{f_{\alpha}\right\},\left\{\rho_{\beta \alpha}\right\}\right)$, where there is a mapping $U_{\alpha} \mapsto U_{\alpha}^{\prime} \in\left\{U_{\alpha^{\prime}}^{\prime}\right\}$, with respect to which $f_{\alpha}: \widehat{U_{\alpha}} \rightarrow \widehat{U_{\alpha}^{\prime}}, \rho_{\beta \alpha}$ : $T\left(U_{\alpha}, U_{\beta}\right) \rightarrow T\left(U_{\alpha}^{\prime}, U_{\beta}^{\prime}\right)$, such that

(a) $\phi_{\rho_{\beta \alpha}(\xi)} \circ f_{\alpha}(x)=f_{\beta} \circ \phi_{\xi}(x)$ for any $\alpha, \beta$, where $\xi \in T\left(U_{\alpha}, U_{\beta}\right), x \in \operatorname{Domain}\left(\phi_{\xi}\right)$.

(b) $\rho_{\gamma \alpha}(\eta \circ \xi(x))=\rho_{\gamma \beta}(\eta) \circ \rho_{\beta \alpha}(\xi)\left(f_{\alpha}(x)\right)$ for any $\alpha, \beta, \gamma$, where $\xi \in T\left(U_{\alpha}, U_{\beta}\right), \eta \in$ $T\left(U_{\beta}, U_{\gamma}\right)$, and $x \in \phi_{\xi}^{-1}\left(\operatorname{Domain}\left(\phi_{\eta}\right)\right)$. Note that $f_{\alpha}(x) \in \phi_{\rho_{\beta \alpha}(\xi)}^{-1}\left(\operatorname{Domain}\left(\phi_{\rho_{\gamma \beta}(\eta)}\right)\right.$, which follows from the equations in (a) and the assumption that $x \in \phi_{\xi}^{-1}\left(\right.$ Domain $\left.\left(\phi_{\eta}\right)\right)$.

Proof Any homomorphism from $\Gamma\left\{U_{\alpha}\right\}$ to $\Gamma\left\{U_{\alpha^{\prime}}^{\prime}\right\}$ will induce a continuous map between the corresponding spaces of units, $\bigsqcup_{\alpha} \widehat{U_{\alpha}}$ and $\bigsqcup_{\alpha^{\prime}} \widehat{U_{\alpha^{\prime}}^{\prime}}$. Since each $\widehat{U_{\alpha}}$ is connected, this map determines a mapping $U_{\alpha} \mapsto U_{\alpha}^{\prime} \in\left\{U_{\alpha^{\prime}}^{\prime}\right\}$ by the rule that $\widehat{U_{\alpha}}$ is being mapped into $\widehat{U_{\alpha}^{\prime}}$. We define $f_{\alpha}: \widehat{U_{\alpha}} \rightarrow \widehat{U_{\alpha}^{\prime}}$ to be the restriction of this map to $\widehat{U_{\alpha}}$.

Since a groupoid homomorphism commutes with the mapping $\omega$, it follows that the image of any $(\xi, x) \in T\left(U_{\alpha}, U_{\beta}\right) \times \widehat{U_{\alpha}}$ under the homomorphism is $\left(\xi^{\prime}, f_{\alpha}(x)\right)$ for some $\xi^{\prime} \in T\left(U_{\alpha}^{\prime}, U_{\beta}^{\prime}\right)$. We define $\rho_{\beta \alpha}: T\left(U_{\alpha}, U_{\beta}\right) \rightarrow T\left(U_{\alpha}^{\prime}, U_{\beta}^{\prime}\right)$ by setting $\rho_{\beta \alpha}(\xi)=\xi^{\prime}$. Then the equations in (a) are a consequence of the fact that the homomorphism commutes with the mapping $\omega$. The 
equations in (b) are simply another way of saying that the homomorphism commutes with composition in the groupoid.

Thus we have shown that any homomorphism from $\Gamma\left\{U_{\alpha}\right\}$ to $\Gamma\left\{U_{\alpha^{\prime}}^{\prime}\right\}$ gives rise to a $\left(\left\{f_{\alpha}\right\},\left\{\rho_{\beta \alpha}\right\}\right)$ with the claimed properties, such that it may be written $(\xi, x) \mapsto\left(\rho_{\beta \alpha}(\xi), f_{\alpha}(x)\right)$ for any $(\xi, x) \in T\left(U_{\alpha}, U_{\beta}\right) \times \widehat{U_{\alpha}}$. On the other hand, any such a $\left(\left\{f_{\alpha}\right\},\left\{\rho_{\beta \alpha}\right\}\right)$ with the claimed properties defines a homomorphism from $\Gamma\left\{U_{\alpha}\right\}$ to $\Gamma\left\{U_{\alpha^{\prime}}^{\prime}\right\}$ in this way, even without the assumption that each $\widehat{U_{\alpha}}$ is connected.

We follow up with a few remarks.

\section{Remark 2.2.2}

(1) In this paper, we shall only consider homomorphisms from $\Gamma\left\{U_{\alpha}\right\}$ to $\Gamma\left\{U_{\alpha^{\prime}}^{\prime}\right\}$ which are in the form $\left(\left\{f_{\alpha}\right\},\left\{\rho_{\beta \alpha}\right\}\right)$. By Lemma 2.2.1, these will cover all the homomorphisms from $\Gamma\left\{U_{\alpha}\right\}$ to $\Gamma\left\{U_{\alpha^{\prime}}^{\prime}\right\}$ if each local chart $\widehat{U_{\alpha}}$ is connected.

(2) For any $\xi_{12}, \xi_{23}, \cdots, \xi_{n-1, n}$, we denote by $\xi_{12}^{\prime}, \xi_{23}^{\prime}, \cdots, \xi_{n-1, n}^{\prime}$ their images under the mappings $\left\{\rho_{\beta \alpha}\right\}$. Then the equations in Lemma 2.2.1 (a) imply that if $x$ lies in the domain of

$$
\phi_{\xi_{n-1, n}} \circ \cdots \circ \phi_{\xi_{23}} \circ \phi_{\xi_{12}}
$$

then $f_{\alpha}(x)$ lies in the domain of

$$
\phi_{\xi_{n-1, n}^{\prime}} \circ \cdots \circ \phi_{\xi_{23}^{\prime}} \circ \phi_{\xi_{12}^{\prime}}
$$

where $\alpha$ is any index such that $\xi_{12} \in T\left(U_{\alpha}, U_{\beta}\right)$ for some $\beta$. This induces a mapping

$$
\underline{\left\{f_{\alpha}\right\}}: \Lambda\left(\xi_{12}, \xi_{23}, \cdots, \xi_{n-1, n}\right) \rightarrow \Lambda\left(\xi_{12}^{\prime}, \xi_{23}^{\prime}, \cdots, \xi_{n-1, n}^{\prime}\right),
$$

which is defined by the rule that if $x \in \mathbf{a}$, then $f_{\alpha}(x) \in\left\{f_{\alpha}\right\}(\mathbf{a})$. With this understood, the equations in Lemma 2.2.1 (b) may be equivalently written as

$$
\rho_{\gamma \alpha}(\eta \circ \xi(\mathbf{a}))=\rho_{\gamma \beta}(\eta) \circ \rho_{\beta \alpha}(\xi)\left(\underline{\left\{f_{\alpha}\right\}}(\mathbf{a})\right), \quad \forall \mathbf{a} \in \Lambda(\xi, \eta) .
$$

(3) Note that the equations in Lemma 2.2 .1 (b) imply that $\rho_{\alpha \alpha}: T\left(U_{\alpha}, U_{\alpha}\right) \rightarrow T\left(U_{\alpha}^{\prime}, U_{\alpha}^{\prime}\right)$ is in fact a homomorphism from $G_{U_{\alpha}}$ to $G_{U_{\alpha}^{\prime}}$ for each $\alpha$, which will be denoted by $\rho_{\alpha}: G_{U_{\alpha}} \rightarrow$ $G_{U_{\alpha}^{\prime}}$ throughout. Moreover, each $f_{\alpha}$ is $\rho_{\alpha}$-equivariant, and we have

$$
\left(\phi_{\rho_{\beta \alpha}(\xi)}, \lambda_{\rho_{\beta \alpha}(\xi)}\right) \circ\left(f_{\alpha}, \rho_{\alpha}\right)=\left(f_{\beta}, \rho_{\beta}\right) \circ\left(\phi_{\xi}, \lambda_{\xi}\right)
$$

over (Domain $\left(\phi_{\xi}\right)$, Domain $\left(\lambda_{\xi}\right)$ ). (See (2.1.3) for the definition of $\lambda_{\xi}$.) In other words, the set of local 'equivariant' maps $\left\{\left(f_{\alpha}, \rho_{\alpha}\right)\right\}$ is compatible with respect to the 'transformations of local charts'.

A map of orbispaces to be defined in this paper will be a certain equivalence class of groupoid homomorphisms of the form described in Lemma 2.2.1. First of all, it proves to be convenient to introduce 
Definition 2.2.3 A homomorphism $\left(\left\{f_{\alpha}^{(2)}\right\},\left\{\rho_{\beta \alpha}^{(2)}\right\}\right): \Gamma\left\{U_{\alpha}\right\} \rightarrow \Gamma\left\{U_{\alpha^{\prime}}^{\prime}\right\}$ is said to be conjugate to $\left(\left\{f_{\alpha}^{(1)}\right\},\left\{\rho_{\beta \alpha}^{(1)}\right\}\right)$ via $\left\{g_{\alpha}\right\}$, where each $g_{\alpha}$ is an element of $G_{U_{\alpha}^{\prime}}$, if

$$
f_{\alpha}^{(2)}=g_{\alpha} \circ f_{\alpha}^{(1)} \text { and } \rho_{\beta \alpha}^{(2)}(\xi)=g_{\beta} \circ \rho_{\beta \alpha}^{(1)}(\xi) \circ g_{\alpha}^{-1}
$$

are satisfied for all $\alpha, \beta$ and all $\xi \in T\left(U_{\alpha}, U_{\beta}\right)$. (Note that $\# \Lambda\left(g_{\alpha}^{-1}, \rho_{\beta \alpha}^{(1)}(\xi), g_{\beta}\right)=1$ so that the notation $g_{\beta} \circ \rho_{\beta \alpha}^{(1)}(\xi) \circ g_{\alpha}^{-1}$ has no ambiguity.)

For the rest of this paper, we shall make the following harmless assumption on the domain orbispace $X$ : in the atlas of local charts $\mathcal{U}=\left\{U_{i}\right\}$, each $\widehat{U}_{i}$ is connected.

Now suppose $\left(\left\{f_{\alpha}\right\},\left\{\rho_{\beta \alpha}\right\}\right): \Gamma\left\{U_{\alpha}\right\} \rightarrow \Gamma\left\{U_{\alpha^{\prime}}^{\prime}\right\}$ is a homomorphism where $\left\{U_{\alpha}\right\}$ is a cover of $X$. Denote by $f: X \rightarrow X^{\prime}$ the induced map between the underlying spaces. Let $\left\{U_{a}\right\} \subset \mathcal{U},\left\{U_{a^{\prime}}^{\prime}\right\} \subset \mathcal{U}^{\prime}$ be any subsets, with a correspondence $U_{a} \mapsto U_{a}^{\prime} \in\left\{U_{a^{\prime}}^{\prime}\right\}$ which satisfies $f\left(U_{a}\right) \subset U_{a}^{\prime}$. Moreover, $\left\{U_{a}\right\}$ is a refinement of $\left\{U_{\alpha}\right\}$ and is also a cover of $X$.

Lemma 2.2.4 The homomorphism $\left(\left\{f_{\alpha}\right\},\left\{\rho_{\beta \alpha}\right\}\right)$ canonically induces a family of homomorphisms $\left(\left\{f_{a}\right\},\left\{\rho_{b a}\right\}\right): \Gamma\left\{U_{a}\right\} \rightarrow \Gamma\left\{U_{a^{\prime}}^{\prime}\right\}$, which are mutually conjugate in the sense of Definition 2.2.3.

Proof Since $\left\{U_{a}\right\}$ is a refinement of $\left\{U_{\alpha}\right\}$, there is a mapping $\theta: a \mapsto \alpha=\theta(a)$ such that $U_{a} \subset U_{\theta(a)}$. We fix a choice $\theta$ of such mappings to start with. By the assumption that $U_{a} \subset U_{\theta(a)}$ and that each $\widehat{U_{a}}$ is connected, we see that $T\left(U_{a}, U_{\theta(a)}\right)$ is nonempty and Domain $\left(\phi_{\xi}\right)=\widehat{U_{a}}$ for each $\xi \in T\left(U_{a}, U_{\theta(a)}\right)$. On the other hand, from the assumption that $f\left(U_{a}\right) \subset U_{a}^{\prime}$, we deduce that $f\left(U_{a}\right) \subset U_{a}^{\prime} \cap U_{\theta(a)}^{\prime}$, and hence $T\left(U_{a}^{\prime}, U_{\theta(a)}^{\prime}\right) \neq \emptyset$. For each $a$, we pick a $\xi_{a} \in T\left(U_{a}, U_{\theta(a)}\right)$, and then fix a choice $\xi_{a}^{\prime}$ of elements $\xi^{\prime} \in T\left(U_{a}^{\prime}, U_{\theta(a)}^{\prime}\right)$ satisfying

$$
f_{\theta(a)} \circ \phi_{\xi_{a}}\left(\widehat{U}_{a}\right) \subset \text { Range }\left(\phi_{\xi^{\prime}}\right)
$$

Now with a fixed choice of data $\left(\theta,\left\{\xi_{a}\right\},\left\{\xi_{a}^{\prime}\right\}\right)$, we define maps $\left\{f_{a}\right\}, f_{a}: \widehat{U_{a}} \rightarrow \widehat{U_{a}^{\prime}}$, by

$$
f_{a}=\left(\phi_{\xi_{a}^{\prime}}\right)^{-1} \circ f_{\theta(a)} \circ \phi_{\xi_{a}}
$$

and for each $(a, b)$ with $U_{a} \cap U_{b} \neq \emptyset$, define a mapping $\rho_{b a}: T\left(U_{a}, U_{b}\right) \rightarrow T\left(U_{a}^{\prime}, U_{b}^{\prime}\right)$ as follows. For any $\eta \in T\left(U_{a}, U_{b}\right)$, we set $\theta(\eta)=\xi_{b} \circ \eta \circ \xi_{a}^{-1} \in T\left(U_{\theta(a)}, U_{\theta(b)}\right)$ (note that $\left.\# \Lambda\left(\xi_{a}^{-1}, \eta, \xi_{b}\right)=1\right)$, and define

$$
\rho_{b a}(\eta)=\left(\xi_{b}^{\prime}\right)^{-1} \circ \rho_{\theta(b) \theta(a)}(\theta(\eta)) \circ \xi_{a}^{\prime}(x), \quad \forall x \in f_{a}\left(\text { Domain }\left(\phi_{\eta}\right)\right) .
$$

One can easily check that $\phi_{\rho_{b a}(\eta)} \circ f_{a}(x)=f_{b} \circ \phi_{\eta}(x)$ for any $\eta \in T\left(U_{a}, U_{b}\right), x \in$ Domain $\left(\phi_{\eta}\right)$. For $\left(\left\{f_{a}\right\},\left\{\rho_{b a}\right\}\right)$ to be a homomorphism from $\Gamma\left\{U_{a}\right\}$ to $\Gamma\left\{U_{a^{\prime}}^{\prime}\right\}$, it remains to show that

$$
\rho_{c a}(\zeta \circ \eta(\mathbf{a}))=\rho_{c b}(\zeta) \circ \rho_{b a}(\eta)\left(\underline{\left\{f_{a}\right\}}(\mathbf{a})\right)
$$

for any $\eta \in T\left(U_{a}, U_{b}\right), \zeta \in T\left(U_{b}, U_{c}\right)$ and $\mathbf{a} \in \Lambda(\eta, \zeta)$. 
Observe that $\Lambda(\eta, \zeta)$ may be identified with $\Lambda\left(\xi_{a}^{-1}, \eta, \zeta, \xi_{c}\right)=\Lambda\left(\xi_{a}^{-1}, \eta, \xi_{b}, \xi_{b}^{-1}, \zeta, \xi_{c}\right)$ via $\mathbf{a} \mapsto \phi_{\xi_{a}}(\mathbf{a})$. We deduce that

$$
\begin{aligned}
\theta(\zeta \circ \eta(\mathbf{a})) & =\xi_{c} \circ \zeta \circ \eta(\mathbf{a}) \circ \xi_{a}^{-1} \\
& =\xi_{c} \circ \zeta \circ \eta \circ \xi_{a}^{-1}\left(\phi_{\xi_{a}}(\mathbf{a})\right) \\
& =\xi_{c} \circ \zeta \circ \xi_{b}^{-1} \circ \xi_{b} \circ \eta \circ \xi_{a}^{-1}\left(\phi_{\xi_{a}}(\mathbf{a})\right) \\
& =\theta(\zeta) \circ \theta(\eta)(\theta(\mathbf{a}))
\end{aligned}
$$

for any $\mathbf{a} \in \Lambda(\eta, \zeta)$, where $\theta(\mathbf{a})$ stands for the unique element of $\Lambda(\theta(\eta), \theta(\zeta))$ which contains $\phi_{\xi_{a}}(\mathbf{a})$. Now we fix a $z \in \mathbf{a}$, and by (2.2.6), we have

$$
\begin{aligned}
& \rho_{c a}(\zeta \circ \eta(\mathbf{a})) \\
= & \left(\xi_{c}^{\prime}\right)^{-1} \circ \rho_{\theta(c) \theta(a)}(\theta(\zeta \circ \eta(\mathbf{a}))) \circ \xi_{a}^{\prime}\left(f_{a}(z)\right) \\
= & \left(\xi_{c}^{\prime}\right)^{-1} \circ \rho_{\theta(c) \theta(a)}(\theta(\zeta) \circ \theta(\eta)(\theta(\mathbf{a}))) \circ \xi_{a}^{\prime}\left(f_{a}(z)\right) \\
= & \left(\xi_{c}^{\prime}\right)^{-1} \circ \rho_{\theta(c) \theta(b)}(\theta(\zeta)) \circ \rho_{\theta(b) \theta(a)}(\theta(\eta))\left(\underline{\left\{f_{\alpha}\right\}}(\theta(\mathbf{a}))\right) \circ \xi_{a}^{\prime}\left(f_{a}(z)\right) \\
= & \left(\xi_{c}^{\prime}\right)^{-1} \circ \rho_{\theta(c) \theta(b)}(\theta(\zeta)) \circ \rho_{\theta(b) \theta(a)}(\theta(\eta)) \circ \xi_{a}^{\prime}\left(f_{a}(z)\right) \\
= & \left(\xi_{c}^{\prime}\right)^{-1} \circ \rho_{\theta(c) \theta(b)}(\theta(\zeta)) \circ \xi_{b}^{\prime} \circ\left(\xi_{b}^{\prime}\right)^{-1} \circ \rho_{\theta(b) \theta(a)}(\theta(\eta)) \circ \xi_{a}^{\prime}\left(f_{a}(z)\right) \\
= & \left(\left(\xi_{c}^{\prime}\right)^{-1} \circ \rho_{\theta(c) \theta(b)}(\theta(\zeta)) \circ \xi_{b}^{\prime}\left(f_{b}\left(\phi_{\eta}(z)\right)\right)\right) \circ\left(\left(\xi_{b}^{\prime}\right)^{-1} \circ \rho_{\theta(b) \theta(a)}(\theta(\eta)) \circ \xi_{a}^{\prime}\left(f_{a}(z)\right)\right)\left(f_{a}(z)\right) \\
= & \rho_{c b}(\zeta) \circ \rho_{b a}(\eta)\left(\underline{\left\{f_{a}\right\}}(\mathbf{a})\right) .
\end{aligned}
$$

We thus verified (2.2.7), and we conclude that $\left(\left\{f_{a}\right\},\left\{\rho_{b a}\right\}\right): \Gamma\left\{U_{a}\right\} \rightarrow \Gamma\left\{U_{a^{\prime}}^{\prime}\right\}$ is a homomorphism.

It remains to examine the dependence of $\left(\left\{f_{a}\right\},\left\{\rho_{b a}\right\}\right)$ on the choice of $\left(\theta,\left\{\xi_{a}\right\},\left\{\xi_{a}^{\prime}\right\}\right)$. Suppose $\bar{\theta}: a \mapsto \alpha$ is another mapping of indices such that $U_{a} \subset U_{\bar{\theta}(a)}$. For any choice of $\bar{\xi}_{a} \in T\left(U_{a}, U_{\bar{\theta}(a)}\right)$, there is a $\bar{\xi}_{a}^{\prime} \in T\left(U_{a}^{\prime}, U_{\bar{\theta}(a)}^{\prime}\right)$ defined by

$$
\bar{\xi}_{a}^{\prime}=\rho_{\bar{\theta}(a) \theta(a)}\left(\bar{\xi}_{a} \circ \xi_{a}^{-1}\right) \circ \xi_{a}^{\prime}\left(\mathbf{a}_{a}\right)
$$

where $\mathbf{a}_{a} \in \Lambda\left(\xi_{a}^{\prime}, \rho_{\bar{\theta}(a) \theta(a)}\left(\bar{\xi}_{a} \circ \xi_{a}^{-1}\right)\right)$ is the element containing the image of $f_{a}, f_{a}\left(\widehat{U_{a}}\right)$. Then $\bar{\xi}_{a}^{\prime}$ satisfies $(2.2 .4)$ with respect to $\bar{\xi}_{a}$, i.e., $f_{\bar{\theta}(a)} \circ \phi_{\bar{\xi}_{a}}\left(\widehat{U_{a}}\right) \subset$ Range $\left(\phi_{\bar{\xi}_{a}^{\prime}}\right)$. We define

$$
\bar{f}_{a}=\left(\phi_{\bar{\xi}_{a}^{\prime}}\right)^{-1} \circ f_{\bar{\theta}(a)} \circ \phi_{\bar{\xi}_{a}}
$$

and

$$
\bar{\rho}_{b a}(\eta)=\left(\bar{\xi}_{b}^{\prime}\right)^{-1} \circ \rho_{\bar{\theta}(b) \bar{\theta}(a)}(\bar{\theta}(\eta)) \circ \bar{\xi}_{a}^{\prime}(x), \forall x \in \bar{f}_{a}\left(\text { Domain }\left(\phi_{\eta}\right)\right), \eta \in T\left(U_{a}, U_{b}\right),
$$

where $\bar{\theta}(\eta)=\bar{\xi}_{b} \circ \eta \circ \bar{\xi}_{a}^{-1}$. We shall verify that $\bar{f}_{a}=f_{a}, \bar{\rho}_{b a}=\rho_{b a}$.

Observe that $\operatorname{Im}\left(f_{\bar{\theta}(a)} \circ \phi_{\bar{\xi}_{a}}\right) \subset \phi_{\bar{\xi}_{a}^{\prime}}\left(\mathbf{a}_{a}\right)$, thus we have

$$
\begin{aligned}
\bar{f}_{a} & =\left(\phi_{\bar{\xi}_{a}^{\prime}}\right)^{-1} \circ f_{\bar{\theta}(a)} \circ \phi_{\bar{\xi}_{a}} \\
& =\left(\phi_{\rho_{\bar{\theta}(a) \theta(a)}\left(\bar{\xi}_{a} \circ \xi_{a}^{-1}\right) \circ \xi_{a}^{\prime}\left(\mathbf{a}_{a}\right)}\right)^{-1} \circ f_{\bar{\theta}(a)} \circ \phi_{\bar{\xi}_{a}} \\
& =\left(\phi_{\xi_{a}^{\prime}}\right)^{-1} \circ \phi_{\rho_{\theta(a) \bar{\theta}(a)}\left(\xi_{a} \circ \bar{\xi}_{a}^{-1}\right)} \circ f_{\bar{\theta}(a)} \circ \phi_{\bar{\xi}_{a}} \\
& =\left(\phi_{\xi_{a}^{\prime}}\right)^{-1} \circ f_{\theta(a)} \circ \phi_{\xi_{a}}=f_{a} .
\end{aligned}
$$

The verification of $\bar{\rho}_{b a}=\rho_{b a}$ is similar, hence we leave it to the reader. 
Thus the dependence of $\left(\left\{f_{a}\right\},\left\{\rho_{b a}\right\}\right)$ on the choices of $\left(\theta,\left\{\xi_{a}\right\},\left\{\xi_{a}^{\prime}\right\}\right)$ boils down to the dependence on the choices of $\left\{\xi_{a}^{\prime}\right\}$, with constrains in (2.2.4), for some fixed choice of $\left\{\xi_{a}\right\}$. It is easy to see that different choices of $\left\{\xi_{a}^{\prime}\right\}$ differ by a pre-composition by an element $g_{a} \in G_{U_{a}^{\prime}}$ for each index $a$. The resulting homomorphisms are conjugate via $\left\{g_{a}\right\}$. Hence different choices of $\left(\theta,\left\{\xi_{a}\right\},\left\{\xi_{a}^{\prime}\right\}\right)$ will result in mutually conjugate homomorphisms $\left(\left\{f_{a}\right\},\left\{\rho_{b a}\right\}\right)$.

We remark that the family of homomorphisms $\left(\left\{f_{a}\right\},\left\{\rho_{b a}\right\}\right)$ all induce the same map $f: X \rightarrow X^{\prime}$ between the underlying spaces. On the other hand, the process $\left(\left\{f_{\alpha}\right\},\left\{\rho_{\beta \alpha}\right\}\right) \Rightarrow$ $\left(\left\{f_{a}\right\},\left\{\rho_{b a}\right\}\right)$ is obviously transitive.

Lemma 2.2.5 The following is indeed an equivalence relation on the set of all groupoid homomorphisms: two homomorphisms are equivalent if they induce a common family of mutually conjugate homomorphisms in the sense of the preceding lemma.

Proof The only nontrivial part is transitivity, namely, for any homomorphisms $\tau_{1}, \tau_{2}$ and $\tau_{3}$, if $\tau_{1}$ is equivalent to $\tau_{2}$ and $\tau_{2}$ is equivalent to $\tau_{3}$, then $\tau_{1}$ is equivalent to $\tau_{3}$. The proof goes as follows. There are homomorphisms $\sigma_{12}, \sigma_{23}$ which are induced by $\tau_{1}, \tau_{2}$ and $\tau_{2}, \tau_{3}$ respectively. By the transitivity of the process of inducing homomorphisms as described in the preceding lemma, if there is a homomorphism $\kappa$ induced by both $\sigma_{12}$ and $\sigma_{23}$, then $\kappa$ must also be induced by both $\tau_{1}$ and $\tau_{3}$, which implies that $\tau_{1}$ is equivalent to $\tau_{3}$.

Thus the problem boils down to show that $\sigma_{12}, \sigma_{23}$ induce a common family of mutually conjugate homomorphisms. To be more explicit, let us assume $\sigma_{12}: \Gamma\left\{U_{a}\right\} \rightarrow \Gamma\left\{U_{a^{\prime}}^{\prime}\right\}$ and $\sigma_{23}: \Gamma\left\{U_{\alpha}\right\} \rightarrow \Gamma\left\{U_{\alpha^{\prime}}^{\prime}\right\}$. We then pick a cover of $X$, denoted by $\left\{U_{s}\right\}$, where each $U_{s}$ is a connected component of $U_{a} \cap U_{\alpha}$ for some indices $a, \alpha$. Clearly $\left\{U_{s}\right\}$ is a refinement of both $\left\{U_{a}\right\}$ and $\left\{U_{\alpha}\right\}$. On the other hand, there exists a $\left\{U_{s^{\prime}}^{\prime}\right\}$, where each $U_{s^{\prime}}^{\prime}$ is a connected component of $U_{a^{\prime}}^{\prime} \cap U_{\alpha^{\prime}}^{\prime}$ for some indices $a^{\prime}, \alpha^{\prime}$, such that each $U_{s}$ may be assigned with a $U_{s}^{\prime} \in\left\{U_{s^{\prime}}^{\prime}\right\}$ so that $f\left(U_{s}\right) \subset U_{s}^{\prime}$. (Here $f: X \rightarrow X^{\prime}$ is the common map of underlying spaces induced by $\sigma_{12}, \sigma_{23}$.) Now by the preceding lemma, there are homomorphisms $\kappa_{12}, \kappa_{23}$ : $\Gamma\left\{U_{s}\right\} \rightarrow \Gamma\left\{U_{s^{\prime}}^{\prime}\right\}$ induced by $\sigma_{12}, \sigma_{23}$ respectively. Again by the transitivity of the process of inducing homomorphisms as described in the preceding lemma, $\kappa_{12}, \kappa_{23}$ are also induced by $\tau_{2}$, hence must be conjugate to each other. Thus we have shown that $\sigma_{12}, \sigma_{23}$ induce a common family of mutually conjugate homomorphisms.

Definition 2.2.6 A map of orbispaces from $X$ to $X^{\prime}$ is an equivalence class of homomorphisms $\left(\left\{f_{\alpha}\right\},\left\{\rho_{\beta \alpha}\right\}\right): \Gamma\left\{U_{\alpha}\right\} \rightarrow \Gamma\left\{U_{\alpha^{\prime}}^{\prime}\right\}$ in the sense of Lemma 2.2.5, where $\left\{U_{\alpha}\right\}$ is a cover of $X$ by local charts.

Now we conclude this subsection with the proof of Theorem 1.2.

Theorem 2.2.7 With the notion of maps in Definition 2.2.6, the set of orbispaces (satisfying (C1), (C2)) forms a category.

Proof It suffices to show that if $\Phi: X \rightarrow Y, \Psi: Y \rightarrow Z$, then their composition $\Psi \circ \Phi:$ $X \rightarrow Z$ is well-defined and associative. 
First of all, we fix a homomorphism $\tau=\left(\left\{g_{a}\right\},\left\{\eta_{b a}\right\}\right): \Gamma\left\{V_{a}\right\} \rightarrow \Gamma\left\{W_{a^{\prime}}\right\}$ whose equivalence class is the map $\Psi: Y \rightarrow Z$, and we shall prove that the composition of $\Phi$ with $\tau$ is welldefined, which will be denoted by $\tau \circ \Phi: X \rightarrow Z$.

Let $\sigma=\left(\left\{f_{\alpha}\right\},\left\{\rho_{\beta \alpha}\right\}\right): \Gamma\left\{U_{\alpha}\right\} \rightarrow \Gamma\left\{V_{\alpha^{\prime}}\right\}$ be any homomorphism which represents the map $\Phi: X \rightarrow Y$. Denote by $f: X \rightarrow Y$ the induced map between the underlying spaces. We consider the set $\left\{U_{i}\right\}$ of all connected components of $U_{\alpha} \cap f^{-1}\left(V_{a}\right)$ for all $\alpha, a$. There is a mapping $\theta$ between the indices $\{i\}$ and $\{\alpha\}, \theta: i \mapsto \alpha=\theta(i)$, such that $U_{i}$ is a connected component of $U_{\theta(i)} \cap f^{-1}\left(V_{a}\right)$ for some index $a$. The mapping $\theta$ defines the cover $\left\{U_{i}\right\}$ as a refinement of $\left\{U_{\alpha}\right\}$. On the other hand, we fix a correspondence $i \mapsto a=\hat{\theta}(i)$ where the indices $i$ and $\hat{\theta}(i)$ satisfy the condition that $U_{i}$ is a connected component of $U_{\alpha} \cap f^{-1}\left(V_{\hat{\theta}(i)}\right)$ for some index $\alpha$. We set $\left\{V_{i^{\prime}}\right\}=\left\{V_{\hat{\theta}(i)}\right\}$, and assign $V_{i}=V_{\hat{\theta}(i)}$ to $U_{i}$ for each index $i$, which verifies the condition $f\left(U_{i}\right) \subset V_{i}$. Now by Lemma 2.2.4, $\sigma$ induces a family of mutually conjugate homomorphisms from $\Gamma\left\{U_{i}\right\}$ to $\Gamma\left\{V_{i^{\prime}}\right\}$. Let $\sigma^{\prime}=\left(\left\{f_{i}\right\},\left\{\rho_{j i}\right\}\right)$ be one of the homomorphisms. We define the composition $\tau \circ \Phi: X \rightarrow Z$ to be the equivalence class of the composition of $\sigma^{\prime}$ with $\tau, \tau \circ \sigma^{\prime}=\left(\left\{h_{i}\right\},\left\{\delta_{j i}\right\}\right): \Gamma\left\{U_{i}\right\} \rightarrow \Gamma\left\{W_{i^{\prime}}\right\}$, where each $U_{i}$ is assigned with $W_{i}=W_{\hat{\theta}(i)}, h_{i}=g_{\hat{\theta}(i)} \circ f_{i}$, and $\delta_{j i}=\eta_{\hat{\theta}(j) \hat{\theta}(i)} \circ \rho_{j i}$.

The map $\tau \circ \Phi$ does not depend on the various choices made in the construction, hence it is well-defined. First, it is independent of the choice of $\sigma^{\prime}$, because a different one is conjugate to $\sigma^{\prime}$ which results in a conjugate composition with $\tau$. In particular, the choice of the mapping $\theta$ is irrelevant here. Second, let's examine the dependence on the choice of $V_{i}$ made via the mapping $i \mapsto a=\hat{\theta}(i)$. Suppose we have two choices $\hat{\theta}_{1}$ and $\hat{\theta}_{2}$, which give rise to $\left\{V_{i, 1}\right\}$ and $\left\{V_{i, 2}\right\}$ by $V_{i, 1}=V_{\hat{\theta}_{1}(i)}$ and $V_{i, 2}=V_{\hat{\theta}_{2}(i)}$. Let $\sigma_{l}^{\prime}=\left(\left\{f_{i, l}\right\},\left\{\rho_{j i, l}\right\}\right): \Gamma\left\{U_{i}\right\} \rightarrow \Gamma\left\{V_{i^{\prime}, l}\right\}$, $l=1,2$, be a choice of the corresponding homomorphisms induced by $\sigma$, which is defined by (2.2.5), (2.2.6) for some choices of $\xi_{i, l}=\xi_{i} \in T\left(U_{i}, U_{\theta(i)}\right)$, and $\xi_{i, l}^{\prime} \in T\left(V_{i, l}, V_{\theta(i)}\right)$ satisfying (2.2.4). Observe that there is an $\mathbf{a} \in \Lambda\left(\xi_{i, 1}^{\prime},\left(\xi_{i, 2}^{\prime}\right)^{-1}\right)$ containing $\phi_{\xi_{i, 1}^{\prime}}^{-1}\left(\operatorname{Im}\left(f_{\theta(i)} \circ \xi_{i}\right)\right)$. We define $\zeta_{i}=\left(\xi_{i, 2}^{\prime}\right)^{-1} \circ \xi_{i, 1}^{\prime}(\mathbf{a}) \in T\left(V_{i, 1}, V_{i, 2}\right)$. Then by (2.2.5), (2.2.6), we have $f_{i, 2}=\zeta_{i} \circ f_{i, 1}$ and $\rho_{j i, 2}=\zeta_{j} \circ \rho_{j i, 1} \circ \zeta_{i}^{-1}$. The corresponding compositions with $\tau, \tau \circ \sigma_{l}^{\prime}=\left(\left\{h_{i, l}\right\},\left\{\delta_{j i, l}\right\}\right)$ : $\Gamma\left\{U_{i}\right\} \rightarrow \Gamma\left\{W_{i^{\prime}, l}\right\}, l=1,2$, which are related by $h_{i, 2}=\zeta_{i}^{\prime} \circ h_{i, 1}$ and $\delta_{j i, 2}=\zeta_{j}^{\prime} \circ \delta_{j i, 1} \circ\left(\zeta_{i}^{\prime}\right)^{-1}$ with $\zeta_{i}^{\prime}=\eta_{\hat{\theta}_{2}(i) \hat{\theta}_{1}(i)}\left(\zeta_{i}\right) \in T\left(W_{i, 1}, W_{i, 2}\right)$, are equivalent homomorphisms. Hence the choice on $\left\{V_{i}\right\}$ is also irrelevant. Finally, suppose $\sigma$ is replaced by a $\sigma_{1}$ which is induced by $\sigma$. Then the corresponding induced homomorphism $\sigma_{1}^{\prime}$, which is from $\Gamma\left\{U_{x}\right\}$ to $\Gamma\left\{V_{i^{\prime}}\right\}$ where $\left\{U_{x}\right\}$ is a refinement of $\left\{U_{i}\right\}$ through a mapping $x \mapsto i=\theta^{\prime}(x)$, and each $U_{x}$ is assigned with $V_{x}=V_{\theta^{\prime}(x)}$, is induced by $\sigma^{\prime}: \Gamma\left\{U_{i}\right\} \rightarrow \Gamma\left\{V_{i^{\prime}}\right\}$. It is easily seen that $\tau \circ \sigma_{1}^{\prime}$ is induced by $\tau \circ \sigma^{\prime}$, so that the choice of $\sigma$ is irrelevant. Hence the map $\tau \circ \Phi$ is well-defined.

We define $\Psi \circ \Phi=\tau \circ \Phi$. In order to see that $\Psi \circ \Phi$ is independent of the choice on $\tau$, we replace $\tau$ in the construction of $\tau \circ \Phi$ by a homomorphism $\tau_{1}$ which is induced by $\tau$, say via the data $\left(\iota,\left\{\xi_{e}\right\},\left\{\xi_{e}^{\prime}\right\}\right)$. Here $\tau_{1}$ is from $\Gamma\left\{V_{e}\right\}$ to $\Gamma\left\{W_{e^{\prime}}\right\}$, where $\left\{V_{e}\right\}$ is a refinement of $\left\{V_{a}\right\}$ via a mapping $e \mapsto a=\iota(e)$. Now we let $\left\{U_{x}\right\}$ be the set of connected components of $U_{\alpha} \cap f^{-1}\left(V_{e}\right)$ for all indices $\alpha, e$. We define a refinement relation between $\left\{U_{x}\right\}$ and $\left\{U_{i}\right\}$ by the following rule: if $U_{x}$ is a connected component of $U_{\alpha} \cap f^{-1}\left(V_{e}\right)$, then $U_{i}$ is the corresponding connected component of $U_{\alpha} \cap f^{-1}\left(V_{\iota(e)}\right)$ such that $U_{x} \subset U_{i}$. Denote the corresponding mapping of indices by $x \mapsto i=\jmath(x)$. We assign each $U_{x}$ with $V_{x}$, where $V_{x}=V_{\hat{\theta}(x)}$ for some choice of mapping $x \mapsto e=\hat{\theta}(x)$. Then we assign each $U_{i}$ with $V_{i}=V_{\iota(\hat{\theta}(x))}$. With these preparations, 
we observe that a homomorphism $\sigma_{1}^{\prime}: \Gamma\left\{U_{x}\right\} \rightarrow \Gamma\left\{V_{x^{\prime}}\right\}$ induced by $\sigma$ may be regarded as a homomorphism induced by the induced homomorphism $\sigma^{\prime}: \Gamma\left\{U_{i}\right\} \rightarrow \Gamma\left\{V_{i^{\prime}}\right\}$ of $\sigma$, say via the data $\left(\jmath,\left\{\xi_{x}\right\},\left\{\xi_{x}^{\prime}\right\}\right)$ where one may arrange to have $\xi_{x}^{\prime}=\xi_{\hat{\theta}(x)}$ for some $\sigma_{1}^{\prime}$. Now it is easy to see that $\tau_{1} \circ \sigma_{1}^{\prime}$ is induced by $\tau \circ \sigma^{\prime}$ via the data $\left(\jmath,\left\{\xi_{x}\right\},\left\{\xi_{\hat{\theta}(x)}^{\prime}\right\}\right)$. Thus we have verified that the composition $\Psi \circ \Phi$ is well-defined.

The compositions are associative: $\tau \circ(\Psi \circ \Phi)=(\tau \circ \Psi) \circ \Phi$ and $\Xi \circ(\Psi \circ \Phi)=(\Xi \circ \Psi) \circ \Phi$, which is clear from the nature of construction.

\section{Structure of MAPping SPACES}

\subsection{SOME PRELIMINARY LEMMAS}

Let $\sigma=\left(\left\{f_{\alpha}\right\},\left\{\rho_{\beta \alpha}\right\}\right): \Gamma\left\{U_{\alpha}\right\} \rightarrow \Gamma\left\{U_{\alpha^{\prime}}^{\prime}\right\}$ be any homomorphism where $\left\{U_{\alpha}\right\}$ is a cover of $X$. We set

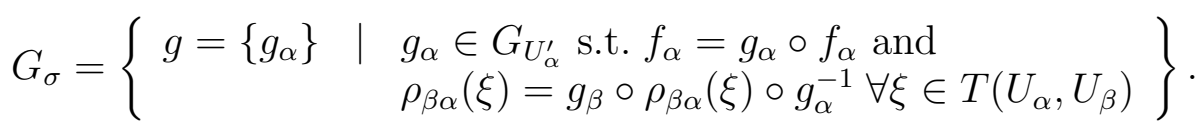

The set $G_{\sigma}$ is naturally a group under $\left\{g_{\alpha}\right\}\left\{h_{\alpha}\right\}=\left\{g_{\alpha} h_{\alpha}\right\}$. We call $G_{\sigma}$ the isotropy group of $\sigma$. Denote by $f: X \rightarrow X^{\prime}$ the induced map of $\sigma$ between the underlying spaces. For each connected component $X_{i}$ of $X$, we pick a $q_{i} \in f\left(X_{i}\right)$, assuming $q_{i} \in U_{\alpha_{i}}^{\prime}$ for some index $\alpha_{i}$. Then there is an injective homomorphism $G_{\sigma} \rightarrow \prod_{i} G_{q_{i}}$ defined by $\left\{g_{\alpha}\right\} \mapsto \prod_{i} g_{\alpha_{i}}$, where $G_{q_{i}}$ stands for the isotropy group of $q_{i}$.

Lemma 3.1.1 To any pair $(\sigma, \tau)$ of equivalent homomorphisms, there is associated a set $\Gamma_{\sigma \tau}$ with the following significance:

(a) Each $\gamma \in \Gamma_{\sigma \tau}$ is assigned with an isomorphism $\epsilon(\gamma): G_{\tau} \rightarrow G_{\sigma}$.

(b) $\Gamma_{\sigma \sigma}$ is canonically identified with $G_{\sigma}$, under which $\epsilon(g)=\operatorname{Ad}(g)$ for any $g \in G_{\sigma}$.

(c) There are mappings $\Gamma_{\sigma \tau} \times \Gamma_{\tau \kappa} \rightarrow \Gamma_{\sigma \kappa}$, denoted by $\left(\gamma_{2}, \gamma_{1}\right) \mapsto \gamma_{2} \circ \gamma_{1}$, which are associative and satisfy $\epsilon\left(\gamma_{2} \circ \gamma_{1}\right)=\epsilon\left(\gamma_{2}\right) \circ \epsilon\left(\gamma_{1}\right)$, and when restricted to $\Gamma_{\sigma \sigma}$, coincide with the multiplication in $G_{\sigma}$ under the canonical identification $\Gamma_{\sigma \sigma}=G_{\sigma}$.

(d) The actions $G_{\sigma} \times \Gamma_{\sigma \tau} \rightarrow \Gamma_{\sigma \tau}$ and $\Gamma_{\sigma \tau} \times G_{\tau} \rightarrow \Gamma_{\sigma \tau}$ on $\Gamma_{\sigma \tau}$ are transitive.

(e) $\gamma \circ g=\epsilon(\gamma)(g) \circ \gamma$ holds for any $g \in G_{\tau}$ and $\gamma \in \Gamma_{\sigma \tau}$.

Proof Let $\tau=\left(\left\{f_{a}\right\},\left\{\rho_{b a}\right\}\right): \Gamma\left\{U_{a}\right\} \rightarrow \Gamma\left\{U_{a^{\prime}}^{\prime}\right\}$ and $\sigma=\left(\left\{f_{\alpha}\right\},\left\{\rho_{\beta \alpha}\right\}\right): \Gamma\left\{U_{\alpha}\right\} \rightarrow \Gamma\left\{U_{\alpha^{\prime}}^{\prime}\right\}$. We shall first prove the lemma for the special case where $\tau$ is induced by $\sigma$, and for any index $\alpha$, there is a subset $\left\{U_{a} \mid a \in I_{\alpha}\right\}$ of $\left\{U_{a}\right\}$ such that

$$
U_{\alpha}=\bigcup_{a \in I_{\alpha}} U_{a} .
$$

In this case, we consider the set $\bar{\Gamma}_{\sigma \tau}$, which consists of all $\left(\theta,\left\{\xi_{a}\right\},\left\{\xi_{a}^{\prime}\right\}\right)$, where $\theta: a \mapsto \alpha$ is a mapping of indices satisfying $U_{a} \subset U_{\theta(a)}$, and $\xi_{a} \in T\left(U_{a}, U_{\theta(a)}\right), \xi_{a}^{\prime} \in T\left(U_{a}^{\prime}, U_{\theta(a)}^{\prime}\right)$, such that the following equations

$$
f_{a}=\left(\phi_{\xi_{a}^{\prime}}\right)^{-1} \circ f_{\theta(a)} \circ \phi_{\xi_{a}}, \quad \rho_{b a}(\eta)=\left(\xi_{b}^{\prime}\right)^{-1} \circ\left(\rho_{\theta(b) \theta(a)} \circ \theta_{b a}(\eta)\right) \circ \xi_{a}^{\prime}(x),
$$


are satisfied, where $\theta_{b a}: T\left(U_{a}, U_{b}\right) \rightarrow T\left(U_{\theta(a)}, U_{\theta(b)}\right)$ is defined by $\eta \mapsto \xi_{b} \circ \eta \circ \xi_{a}^{-1}$, and $x \in f_{a}$ (Domain $\left(\phi_{\eta}\right)$ ), cf. equations (2.2.5) and (2.2.6). The set $\Gamma_{\sigma \tau}$ is $\bar{\Gamma}_{\sigma \tau}$ modulo the following equivalence relation: $\left(\theta_{1},\left\{\xi_{a, 1}\right\},\left\{\xi_{a, 1}^{\prime}\right\}\right)$ and $\left(\theta_{2},\left\{\xi_{a, 2}\right\},\left\{\xi_{a, 2}^{\prime}\right\}\right)$ are equivalent if and only if

$$
\rho_{\theta_{2}(a) \theta_{1}(a)}\left(\xi_{a, 2} \circ \xi_{a, 1}^{-1}\right)=\xi_{a, 2}^{\prime} \circ\left(\xi_{a, 1}^{\prime}\right)^{-1}(x), \forall x \in f_{a}\left(\widehat{U_{a}}\right) .
$$

One can easily verify that (3.1.4) indeed defines an equivalence relation, and that as in the proof of Lemma 2.2.4, (3.1.3) is preserved under this equivalence. When $\tau$ and $\sigma$ are identical, $G_{\tau}$ is canonically identified with $\Gamma_{\tau \tau}$ by the correspondence $\left\{g_{a}\right\} \mapsto\left(I d,\{1\},\left\{g_{a}\right\}\right)$. Suppose $\kappa: \Gamma\left\{U_{i}\right\} \rightarrow \Gamma\left\{U_{i^{\prime}}^{\prime}\right\}$ is a homomorphism induced by $\tau$. We define the mappings $\Gamma_{\sigma \tau} \times \Gamma_{\tau \kappa} \rightarrow \Gamma_{\sigma \kappa}$, denoted by $\left(\gamma_{2}, \gamma_{1}\right) \mapsto \gamma_{2} \circ \gamma_{1}$, to be the ones induced by

$$
\left(\left(\theta,\left\{\xi_{a}\right\},\left\{\xi_{a}^{\prime}\right\}\right),\left(\iota,\left\{\eta_{i}\right\},\left\{\eta_{i}^{\prime}\right\}\right)\right) \mapsto\left(\theta \circ \iota,\left\{\xi_{\iota(i)} \circ \eta_{i}\right\},\left\{\xi_{\iota(i)}^{\prime} \circ \eta_{i}^{\prime}\right\}\right) .
$$

It is a routine exercise to check that the equivalence defined by (3.1.4) is preserved under (3.1.5). Hence the mappings $\Gamma_{\sigma \tau} \times \Gamma_{\tau \kappa} \rightarrow \Gamma_{\sigma \kappa}$ are well-defined, which are naturally associative. Under the canonical identification $G_{\tau}=\Gamma_{\tau \tau}$, we have $g_{2} \circ g_{1}=g_{2} g_{1}$ for any $g_{1}, g_{2} \in G_{\tau}$. The action $G_{\sigma} \times \Gamma_{\sigma \tau} \rightarrow \Gamma_{\sigma \tau}$ is thus on the left and the action $\Gamma_{\sigma \tau} \times G_{\tau} \rightarrow \Gamma_{\sigma \tau}$ is on the right. The transitivity of the latter is part of Lemma 2.2.4, and the transitivity of the former follows from the existence of isomorphisms $\epsilon(\gamma): G_{\tau} \rightarrow G_{\sigma}$ which satisfy $\gamma \circ g=\epsilon(\gamma)(g) \circ \gamma$ for any $g \in G_{\tau}$ and $\gamma \in \Gamma_{\sigma \tau}$. The construction of $\epsilon(\gamma): G_{\tau} \rightarrow G_{\sigma}$ will occupy the next four paragraphs.

For each $\gamma \in \Gamma_{\sigma \tau}$, we define the isomorphism $\epsilon(\gamma): G_{\tau} \rightarrow G_{\sigma}$ as follows. First of all, we fix a representative $\bar{\gamma}=\left(\theta,\left\{\xi_{a}\right\},\left\{\xi_{a}^{\prime}\right\}\right)$ of $\gamma$. Now suppose $\left\{g_{a}\right\} \in G_{\tau}$ is any given element. Then for each $U_{\alpha}$, we assign each pair $\left(U_{a}, \eta_{a}\right)$, where $U_{a} \in\left\{U_{a} \mid a \in I_{\alpha}\right\}$ and $\eta_{a} \in T\left(U_{a}, U_{\alpha}\right)$, with an $\eta_{a}^{\prime} \in T\left(U_{a}^{\prime}, U_{\alpha}^{\prime}\right)$ defined by

$$
\eta_{a}^{\prime}=\rho_{\alpha \theta(a)}\left(\eta_{a} \circ \xi_{a}^{-1}\right) \circ \xi_{a}^{\prime}(x), \forall x \in f_{a}\left(\widehat{U_{a}}\right) .
$$

With this understood, we define

$$
g\left(U_{a}, \eta_{a}\right)=\eta_{a}^{\prime} \circ g_{a} \circ\left(\eta_{a}^{\prime}\right)^{-1}(x), \forall x \in f_{a}\left(\widehat{U_{a}}\right) .
$$

Note that $g\left(U_{a}, \eta_{a}\right) \in T\left(U_{\alpha}^{\prime}, U_{\alpha}^{\prime}\right)=G_{U_{\alpha}^{\prime}}$. We shall prove that $g\left(U_{a}, \eta_{a}\right)$ depends only on the index $\alpha$, whose common value is to be denoted by $g_{\alpha}$, and that $\left\{g_{\alpha}\right\}$ is an element of $G_{\sigma}$. We define $\epsilon(\gamma)$ by $\left\{g_{a}\right\} \mapsto\left\{g_{\alpha}\right\}$.

It is easy to see that $g\left(U_{a}, \eta_{a}\right)$ satisfies $\left.g\left(U_{a}, \eta_{a}\right) \circ f_{\alpha}\right|_{\text {Range }{\left(\phi_{\eta_{a}}\right)}}=\left.f_{\alpha}\right|_{\text {Range }\left(\phi_{\eta_{a}}\right)}$. Now let $\left(U_{b}, \eta_{b}\right)$ be another pair such that Range $\left(\phi_{\eta_{a}}\right) \cap$ Range $\left(\phi_{\eta_{b}}\right) \neq \emptyset$. Then $\Lambda\left(\eta_{a}, \eta_{b}^{-1}\right) \neq \emptyset$. Let $\mathbf{a} \in \Lambda\left(\eta_{a}, \eta_{b}^{-1}\right)$ be any element, and $\mathbf{a}^{\prime} \in \Lambda\left(\eta_{a}^{\prime},\left(\eta_{b}^{\prime}\right)^{-1}\right)$ be the corresponding element containing $f_{a}(\mathbf{a})$. Then by the second equation in (3.1.3), we have

$$
\rho_{b a}\left(\eta_{b}^{-1} \circ \eta_{a}(\mathbf{a})\right)=\left(\eta_{b}^{\prime}\right)^{-1} \circ \rho_{\alpha \alpha}\left(\eta_{b} \circ\left(\eta_{b}^{-1} \circ \eta_{a}(\mathbf{a})\right) \circ \eta_{a}^{-1}\right) \circ \eta_{a}^{\prime}\left(\mathbf{a}^{\prime}\right)=\left(\eta_{b}^{\prime}\right)^{-1} \circ \eta_{a}^{\prime}\left(\mathbf{a}^{\prime}\right) .
$$

With this in hand, the following holds on the appropriate components:

$$
\begin{aligned}
& \left(\eta_{b}^{\prime}\right)^{-1} \circ g\left(U_{a}, \eta_{a}\right)=\rho_{b a}\left(\eta_{b}^{-1} \circ \eta_{a}\right) \circ g_{a} \circ\left(\eta_{a}^{\prime}\right)^{-1} \\
= & g_{b} \circ \rho_{b a}\left(\eta_{b}^{-1} \circ \eta_{a}\right) \circ\left(\eta_{a}^{\prime}\right)^{-1}=g_{b} \circ\left(\eta_{b}^{\prime}\right)^{-1}=\left(\eta_{b}^{\prime}\right)^{-1} \circ g\left(U_{b}, \eta_{b}\right),
\end{aligned}
$$


which implies that $g\left(U_{a}, \eta_{a}\right)=g\left(U_{b}, \eta_{b}\right)$. Since $U_{\alpha}=U_{a \in I_{\alpha}} U_{a}$ and $\widehat{U_{\alpha}}$ is connected, we actually proved that $g\left(U_{a}, \eta_{a}\right)$ depends only on $\alpha$, which is to be denoted by $g_{\alpha}$. It is clear that $g_{\alpha} \circ f_{\alpha}=f_{\alpha}$. We have to check that $\rho_{\beta \alpha}(\xi)=g_{\beta} \circ \rho_{\beta \alpha}(\xi) \circ g_{\alpha}^{-1}$ for any $\xi \in T\left(U_{\alpha}, U_{\beta}\right)$.

Given any $\xi \in T_{W}\left(U_{\alpha}, U_{\beta}\right)$, since $U_{\alpha}=\bigcup_{a \in I_{\alpha}} U_{a}$, there must be $a \in I_{\alpha}$ and $b \in I_{\beta}$ such that $U_{a} \cap U_{b} \neq \emptyset$ and has a component contained in $W$, and there exist $\eta_{a} \in T\left(U_{a}, U_{\alpha}\right)$ and $\eta_{b} \in T\left(U_{b}, U_{\beta}\right)$ such that $\Lambda\left(\eta_{a}, \xi, \eta_{b}^{-1}\right) \neq \emptyset$. We set $\eta_{\mathbf{a}}=\eta_{b}^{-1} \circ \xi \circ \eta_{a}(\mathbf{a}), \forall \mathbf{a} \in \Lambda\left(\eta_{a}, \xi, \eta_{b}^{-1}\right)$. Let $\eta_{a}^{\prime} \in T\left(U_{a}^{\prime}, U_{\alpha}^{\prime}\right), \eta_{b}^{\prime} \in T\left(U_{b}^{\prime}, U_{\beta}^{\prime}\right)$ be the elements associated to $\eta_{a}, \eta_{b}$ by (3.1.6). Then we have $\left(\eta_{b}^{\prime}\right)^{-1} \circ \rho_{\beta \alpha}(\xi) \circ \eta_{a}^{\prime}\left(\mathbf{a}^{\prime}\right)=\rho_{b a}\left(\eta_{\mathbf{a}}\right)$, where $\mathbf{a}^{\prime}$ is the component containing $f_{a}(\mathbf{a})$. With (3.1.7), $\rho_{\beta \alpha}(\xi)=g_{\beta} \circ \rho_{\beta \alpha}(\xi) \circ g_{\alpha}^{-1}$ can be easily deduced.

It is easy to see that $\epsilon(\gamma): G_{\tau} \rightarrow G_{\sigma}$ is independent of the choice made on the representative $\bar{\gamma}=\left(\theta,\left\{\xi_{a}\right\},\left\{\xi_{a}^{\prime}\right\}\right)$, and that $\epsilon(\gamma)$ is an isomorphism. The other properties, i.e., (1) $\epsilon(g)=A d(g)$ for any $g \in G_{\tau}$ with $G_{\tau}, \Gamma_{\tau \tau}$ canonically identified, $(2) \epsilon\left(\gamma_{2} \circ \gamma_{1}\right)=\epsilon\left(\gamma_{2}\right) \circ \epsilon\left(\gamma_{1}\right)$, and (3) $\gamma \circ g=\epsilon(\gamma)(g) \circ \gamma$ for any $g \in G_{\tau}$ and $\gamma \in \Gamma_{\sigma \tau}$, can be easily verified from the construction. Thus we have completed the proof of the lemma under the assumption that $\tau$ is induced by $\sigma$ and (3.1.2) is satisfied.

To motivate the construction for the general case, we observe that the mappings $\Gamma_{\sigma \tau} \times$ $\Gamma_{\tau \kappa} \rightarrow \Gamma_{\sigma \kappa}$, where $\tau$ is induced by $\sigma, \kappa$ is induced by $\tau$, and (3.1.2) is satisfied for both pairs, canonically identify $\Gamma_{\sigma \tau}$ with the orbit space $\left(\Gamma_{\sigma \kappa} \times \Gamma_{\tau \kappa}\right) / G_{\kappa}$ via $\gamma \mapsto\left[\gamma \circ \gamma_{0}, \gamma_{0}\right], \forall \gamma_{0} \in \Gamma_{\tau \kappa}$, where the action of $G_{\kappa}$ is given by $\left(\gamma_{1}, \gamma_{2}\right) \cdot g=\left(\gamma_{1} \circ g, \gamma_{2} \circ g\right)$. Now for any pair $(\sigma, \tau)$ of equivalent homomorphisms, let $\kappa$ be a homomorphism induced by both $\tau$ and $\sigma$ such that (3.1.2) is satisfied. We define $\Gamma_{\sigma \tau}$ to be $\Gamma_{\sigma \kappa} \times \Gamma_{\tau \kappa}$ modulo the action of $G_{\kappa}$. Moreover, we define $\epsilon\left(\left[\gamma_{1}, \gamma_{2}\right]\right)=\epsilon\left(\gamma_{1}\right) \circ \epsilon\left(\gamma_{2}\right)^{-1}$. The orbit space $\left(\Gamma_{\sigma \kappa} \times \Gamma_{\tau \kappa}\right) / G_{\kappa}$ for different choices of $\kappa$ can be canonically identified, using the fact that $\gamma \circ g=\epsilon(\gamma)(g) \circ \gamma$ for any $g \in G_{\tau}$ and $\gamma \in \Gamma_{\sigma \tau}$ whenever $\tau$ is induced by $\sigma$ and (3.1.2) is satisfied. On the other hand, for any homomorphism $\kappa$ which is induced by $\tau$ with (3.1.2) satisfied, we define a mapping $\Gamma_{\tau \kappa} \times \Gamma_{\tau \kappa} \rightarrow G_{\kappa}$, denoted by $\left(\gamma_{1}, \gamma_{2}\right) \mapsto \gamma_{1} \times \gamma_{2}$, as follows. For any $\gamma_{1}, \gamma_{2} \in \Gamma_{\tau \kappa}$, we write $\gamma_{1}=\gamma_{0} \circ g_{1}$ and $\gamma_{2}=\gamma_{0} \circ g_{2}$ for some $\gamma_{0} \in \Gamma_{\tau \kappa}, g_{1}, g_{2} \in G_{\kappa}$, and then define $\gamma_{1} \times \gamma_{2}=g_{1}^{-1} g_{2}$. It follows easily that $\gamma_{1} \times \gamma_{2}$ is well-defined and satisfies $\left(\gamma_{1} \circ g_{1}\right) \times\left(\gamma_{2} \circ g_{2}\right)=g_{1}^{-1}\left(\gamma_{1} \times \gamma_{2}\right) g_{2}$. Now to define the mappings $\Gamma_{\sigma \tau} \times \Gamma_{\tau \kappa} \rightarrow \Gamma_{\sigma \kappa}$ for the general case, we take a homomorphism $\zeta$ induced by all $\sigma, \tau, \kappa$, and identify $\Gamma_{\sigma \tau}, \Gamma_{\tau \kappa}$ and $\Gamma_{\sigma \kappa}$ as the orbit space $\Gamma_{\sigma \zeta} \times \Gamma_{\tau \zeta} / G_{\zeta}$, $\Gamma_{\tau \zeta} \times \Gamma_{\kappa \zeta} / G_{\zeta}$ and $\Gamma_{\sigma \zeta} \times \Gamma_{\kappa \zeta} / G_{\zeta}$ respectively. Then for any $\gamma \in \Gamma_{\sigma \tau}, \gamma^{\prime} \in \Gamma_{\tau \kappa}$, we write $\gamma=\left[\gamma_{1}, \gamma_{2}\right], \gamma^{\prime}=\left[\gamma_{3}, \gamma_{4}\right]$, and define $\gamma \circ \gamma^{\prime}=\left[\gamma_{1} \circ\left(\gamma_{2} \times \gamma_{3}\right), \gamma_{4}\right]$. We leave the verifications to the reader. (It is instructive to think $\left[\gamma_{1}, \gamma_{2}\right]$ as $\gamma_{1} \circ \gamma_{2}^{-1}$ and $\gamma_{1} \times \gamma_{2}$ as $\gamma_{1}^{-1} \circ \gamma_{2}$.)

We remark that if we let $U(\Phi)=\{\sigma \mid \sigma$ is a homomorphism representing $\Phi\}$ and $\Gamma(\Phi)=$ $\bigcup_{(\sigma, \tau)} \Gamma_{\sigma \tau}$, with $\alpha, \omega: \Gamma(\Phi) \rightarrow U(\Phi)$ given by $\alpha(\gamma)=\tau, \omega(\gamma)=\sigma, \forall \gamma \in \Gamma_{\sigma \tau}$, then the preceding lemma implies that $\Gamma(\Phi)$ is a groupoid acting on $U(\Phi)$, with the set of orbits $\Gamma(\Phi) \backslash U(\Phi)=\{\Phi\}$.

Lemma 3.1.2 Let $\left(\left\{f_{\alpha}^{(1)}\right\},\left\{\rho_{\beta \alpha}^{(1)}\right\}\right),\left(\left\{f_{\alpha}^{(2)}\right\},\left\{\rho_{\beta \alpha}^{(2)}\right\}\right): \Gamma\left\{U_{\alpha}\right\} \rightarrow \Gamma\left\{U_{\alpha^{\prime}}^{\prime}\right\}$ be any pair of equivalent homomorphisms, where the assignment $U_{\alpha} \mapsto U_{\alpha}^{\prime}$ is common for both of them. Then $\left(\left\{f_{\alpha}^{(1)}\right\},\left\{\rho_{\beta \alpha}^{(1)}\right\}\right),\left(\left\{f_{\alpha}^{(2)}\right\},\left\{\rho_{\beta \alpha}^{(2)}\right\}\right)$ are in fact conjugate to each other. 
Proof By the assumption that $\left(\left\{f_{\alpha}^{(1)}\right\},\left\{\rho_{\beta \alpha}^{(1)}\right\}\right)$ and $\left(\left\{f_{\alpha}^{(2)}\right\},\left\{\rho_{\beta \alpha}^{(2)}\right\}\right)$ are equivalent, there exists a common induced homomorphism $\left(\left\{f_{a}\right\},\left\{\rho_{b a}\right\}\right): \Gamma\left\{U_{a}\right\} \rightarrow \Gamma\left\{U_{a^{\prime}}^{\prime}\right\}$. Without loss of generality, we assume that (3.1.2) is satisfied: for any index $\alpha$, there is a subset $\left\{U_{a} \mid a \in I_{\alpha}\right\}$ of $\left\{U_{a}\right\}$ such that $U_{\alpha}=\bigcup_{a \in I_{\alpha}} U_{a}$.

For $i=1,2$, we fix a set of data $\left(\theta_{i},\left\{\xi_{a, i}\right\},\left\{\xi_{a, i}^{\prime}\right\}\right)$, where $\theta_{i}: a \mapsto \alpha$ is a mapping of indices such that $U_{a} \subset U_{\theta_{i}(a)}$, and $\xi_{a, i} \in T\left(U_{a}, U_{\theta_{i}(a)}\right), \xi_{a, i}^{\prime} \in T\left(U_{a}^{\prime}, U_{\theta_{i}(a)}^{\prime}\right)$ so that

$$
f_{a}=\left(\xi_{a, i}^{\prime}\right)^{-1} \circ f_{\theta_{i}(a)}^{(i)} \circ \xi_{a, i}
$$

and

$$
\rho_{b a}(\eta)=\left(\xi_{b, i}^{\prime}\right)^{-1} \circ \rho_{\theta_{i}(b) \theta_{i}(a)}^{(i)}\left(\xi_{b, i} \circ \eta \circ \xi_{a, i}^{-1}\right) \circ \xi_{a, i}^{\prime}(x) \forall x \in f_{a}\left(\operatorname{Domain}\left(\phi_{\eta}\right)\right), \eta \in T\left(U_{a}, U_{b}\right) .
$$

Now given any $\alpha$ and $a \in I_{\alpha}$, we associate a $\zeta_{a, i}^{\prime} \in T\left(U_{a}^{\prime}, U_{\alpha}^{\prime}\right)$ to each $\zeta_{a} \in T\left(U_{a}, U_{\alpha}\right)$ by $\zeta_{a, i}^{\prime}=\rho_{\alpha \theta_{i}(a)}^{(i)}\left(\zeta_{a} \circ \xi_{a, i}^{-1}\right) \circ \xi_{a, i}^{\prime}(x), \forall x \in f_{a}\left(\widehat{U_{a}}\right)$. It is easy to check that for any $a \in I_{\alpha}, b \in I_{\beta}$, one has

$$
f_{a}=\left(\zeta_{a, i}^{\prime}\right)^{-1} \circ f_{\alpha}^{(i)} \circ \zeta_{a}
$$

and

$$
\rho_{b a}(\eta)=\left(\zeta_{b, i}^{\prime}\right)^{-1} \circ \rho_{\beta \alpha}^{(i)}\left(\zeta_{b} \circ \eta \circ \zeta_{a}^{-1}\right) \circ \zeta_{a, i}^{\prime}(x) \forall x \in f_{a}\left(\text { Domain }\left(\phi_{\eta}\right)\right), \eta \in T\left(U_{a}, U_{b}\right) .
$$

We define $g_{\alpha}\left(\zeta_{a}\right)=\zeta_{a, 2}^{\prime} \circ\left(\zeta_{a, 1}^{\prime}\right)^{-1}(x), \forall x \in f_{a}\left(\widehat{U_{a}}\right)$. Then $g_{\alpha}\left(\zeta_{a}\right) \in T\left(U_{\alpha}^{\prime}, U_{\alpha}^{\prime}\right)=G_{U_{\alpha}^{\prime}}$. Observe that if Range $\left(\phi_{\zeta_{a}}\right) \cap$ Range $\left(\phi_{\zeta_{b}}\right) \neq \emptyset$, then $\Lambda\left(\zeta_{a}, \zeta_{b}^{-1}\right) \neq \emptyset$. We set $\eta_{\mathbf{a}}=\zeta_{b}^{-1} \circ \zeta_{a}(\mathbf{a}) \in$ $T\left(U_{a}, U_{b}\right)$ for any $\mathbf{a} \in \Lambda\left(\zeta_{a}, \zeta_{b}^{-1}\right)$. Then $\rho_{b a}\left(\eta_{\mathbf{a}}\right)=\left(\zeta_{b, i}^{\prime}\right)^{-1} \circ \zeta_{a, i}^{\prime}(x), \forall x \in f_{a}(\mathbf{a})$, for $i=1,2$. This implies that $g_{\alpha}\left(\zeta_{a}\right)=g_{\alpha}\left(\zeta_{b}\right)$ whenever Range $\left(\phi_{\zeta_{a}}\right) \cap$ Range $\left(\phi_{\zeta_{b}}\right) \neq \emptyset$. But $\bigcup_{a \in I_{\alpha}} U_{a}=$ $U_{\alpha}$, and $\widehat{U_{\alpha}}$ is connected, so $g_{\alpha}\left(\zeta_{a}\right)$ is in fact independent of $\zeta_{a}$. We define $g_{\alpha}=g_{\alpha}\left(\zeta_{a}\right)$.

It remains to verify that $\left(\left\{f_{\alpha}^{(2)}\right\},\left\{\rho_{\beta \alpha}^{(2)}\right\}\right)$ is conjugate to $\left(\left\{f_{\alpha}^{(1)}\right\},\left\{\rho_{\beta \alpha}^{(1)}\right\}\right)$ via $\left\{g_{\alpha}\right\}$. First, $f_{\alpha}^{(2)}=g_{\alpha} \circ f_{\alpha}^{(1)}$ is clear from the construction of $g_{\alpha}$. Second, given any $\xi \in T_{W}\left(U_{\alpha}, U_{\beta}\right)$, since $U_{\alpha}=\bigcup_{a \in I_{\alpha}} U_{a}$, there must be $a \in I_{\alpha}$ and $b \in I_{\beta}$ such that $U_{a} \cap U_{b} \neq \emptyset$ and has a connected component contained in $W$, and there exist $\zeta_{a} \in T\left(U_{a}, U_{\alpha}\right)$ and $\zeta_{b} \in T\left(U_{b}, U_{\beta}\right)$ such that $\Lambda\left(\zeta_{a}, \xi, \zeta_{b}^{-1}\right) \neq \emptyset$. We set $\eta_{\mathbf{a}}=\zeta_{b}^{-1} \circ \xi \circ \zeta_{a}(\mathbf{a}), \forall \mathbf{a} \in \Lambda\left(\zeta_{a}, \xi, \zeta_{b}^{-1}\right)$. Then $\rho_{b a}\left(\eta_{\mathbf{a}}\right)=$ $\left(\zeta_{b, i}^{\prime}\right)^{-1} \circ \rho_{\beta \alpha}^{(i)}(\xi) \circ \zeta_{a, i}^{\prime}(x), \forall x \in f_{a}(\mathbf{a})$, for $i=1,2$. We deduce easily from these equations that $\rho_{\beta \alpha}^{(2)}(\xi)=g_{\beta} \circ \rho_{\beta \alpha}^{(1)}(\xi) \circ g_{\alpha}^{-1}$. Hence $\left(\left\{f_{\alpha}^{(2)}\right\},\left\{\rho_{\beta \alpha}^{(2)}\right\}\right)$ is conjugate to $\left(\left\{f_{\alpha}^{(1)}\right\},\left\{\rho_{\beta \alpha}^{(1)}\right\}\right)$ via $\left\{g_{\alpha}\right\}$.

Lemma 3.1.3 Let $\Phi: X \rightarrow X^{\prime}$ be any map of orbispaces. Suppose there are $\left\{U_{\alpha}\right\},\left\{U_{\alpha^{\prime}}^{\prime}\right\}$ of local charts with the following significance:

- $\left\{U_{\alpha}\right\}$ is a cover of $X$.

- There is a correspondence $U_{\alpha} \mapsto U_{\alpha}^{\prime} \in\left\{U_{\alpha^{\prime}}^{\prime}\right\}$ such that for each $U_{\alpha}$, the restriction of $\Phi$ to the open subspace $U_{\alpha}$ of $X$ is represented by a pair $\left(f_{\alpha}, \rho_{\alpha}\right):\left(\widehat{U}_{\alpha}, G_{U_{\alpha}}\right) \rightarrow$ $\left(\widehat{U_{\alpha}^{\prime}}, G_{U_{\alpha}^{\prime}}\right)$ where $f_{\alpha}$ is $\rho_{\alpha}$-equivariant.

Then there are mappings $\rho_{\beta \alpha}: T\left(U_{\alpha}, U_{\beta}\right) \rightarrow T\left(U_{\alpha}^{\prime}, U_{\beta}^{\prime}\right)$ with $\rho_{\alpha \alpha}=\rho_{\alpha}$ such that $\left(\left\{f_{\alpha}\right\},\left\{\rho_{\beta \alpha}\right\}\right)$ : $\Gamma\left\{U_{\alpha}\right\} \rightarrow \Gamma\left\{U_{\alpha^{\prime}}^{\prime}\right\}$ is a homomorphism whose equivalence class is the given map $\Phi$.

Proof We pick a homomorphism $\tau=\left(\left\{f_{a}\right\},\left\{\rho_{b a}\right\}\right): \Gamma\left\{U_{a}\right\} \rightarrow \Gamma\left\{U_{a^{\prime}}^{\prime}\right\}$ whose equivalence class is $\Phi: X \rightarrow X^{\prime}$, where we may assume, by passing to an induced homomorphism, that 
(3.1.2) is satisfied: for each $\alpha$, there is a subset $\left\{U_{a} \mid a \in I_{\alpha}\right\} \subset\left\{U_{a}\right\}$ such that $U_{\alpha}=\bigcup_{a \in I_{\alpha}} U_{a}$. The restriction of $\tau$ to $U_{\alpha}$, denoted by $\tau_{\alpha}=\left(\left\{f_{a}\right\},\left\{\rho_{b a}\right\}\right)$ where $a, b \in I_{\alpha}$, represents the restriction of $\Phi$ to the open subspace $U_{\alpha}$. On the other hand, the restriction of $\Phi$ to $U_{\alpha}$ is also represented by $\left(f_{\alpha}, \rho_{\alpha}\right)$. We apply Lemma 2.2.4 first to obtain an induced homomorphism of $\left(f_{\alpha}, \rho_{\alpha}\right)$ from $\Gamma\left\{U_{a}\right\}$ to $\Gamma\left\{U_{a^{\prime}}^{\prime}\right\}$ where each $U_{a} \mapsto U_{a}^{\prime}$, then apply Lemma 3.1.2 to conclude that $\tau_{\alpha}=\left(\left\{f_{a}\right\},\left\{\rho_{b a}\right\}\right)$ is conjugate to the induced one, so that $\tau_{\alpha}$ is induced by $\left(f_{\alpha}, \rho_{\alpha}\right)$ also. Hence there are $\xi_{a, \alpha} \in T\left(U_{a}, U_{\alpha}\right), \xi_{a, \alpha}^{\prime} \in T\left(U_{a}^{\prime}, U_{\alpha}^{\prime}\right)$, where $a \in I_{\alpha}$, such that

$$
f_{a}=\left(\phi_{\xi_{a, \alpha}^{\prime}}\right)^{-1} \circ f_{\alpha} \circ \phi_{\xi_{a, \alpha}} \forall a \in I_{\alpha},
$$

and for any $\eta \in T\left(U_{a}, U_{b}\right), a, b \in I_{\alpha}$,

$$
\rho_{b a}(\eta)=\left(\xi_{b, \alpha}^{\prime}\right)^{-1} \circ \rho_{\alpha}\left(\xi_{b, \alpha} \circ \eta \circ \xi_{a, \alpha}^{-1}\right) \circ \xi_{a, \alpha}^{\prime}(x) \forall x \in f_{a}\left(\text { Domain }\left(\phi_{\eta}\right)\right) .
$$

Note that if we replace $\xi_{a, \alpha}$ by $g \circ \xi_{a, \alpha}$ for any $g \in G_{U_{\alpha}}$ and correspondingly replace $\xi_{a, \alpha}^{\prime}$ by $\rho_{\alpha}(g) \circ \xi_{a, \alpha}^{\prime},(3.1 .8)$ and (3.1.9) continue to hold. In other words, (3.1.8) and (3.1.9) hold with $\xi_{a, \alpha}$ allowed to be any element in $T\left(U_{a}, U_{\alpha}\right)$ as long as $\xi_{a, \alpha}^{\prime}$ is chosen by the correspondence $\xi_{a, \alpha} \mapsto \xi_{a, \alpha}^{\prime}$ described above.

Now suppose $U_{\alpha}, U_{\beta} \in\left\{U_{\alpha}\right\}$ have nonempty intersection. Given any $\zeta \in T\left(U_{\alpha}, U_{\beta}\right)$, since $U_{\alpha}=\bigcup_{a \in I_{\alpha}} U_{a}, U_{\beta}=\bigcup_{b \in I_{\beta}} U_{b}$, there are $a \in I_{\alpha}, b \in I_{\beta}$, with $\xi_{a, \alpha} \in T\left(U_{a}, U_{\alpha}\right)$, $\xi_{b, \beta} \in T\left(U_{b}, U_{\beta}\right)$, and $\eta \in T\left(U_{a}, U_{b}\right)$, such that $\zeta=\xi_{b, \beta} \circ \eta \circ \xi_{a, \alpha}^{-1}$. We define

$$
\rho_{\beta \alpha}(\zeta)=\xi_{b, \beta}^{\prime} \circ \rho_{b a}(\eta) \circ\left(\xi_{a, \alpha}^{\prime}\right)^{-1}(x) \forall x \in \phi_{\xi_{a, \alpha}^{\prime}}\left(f_{a}\left(\text { Domain }\left(\phi_{\eta}\right)\right)\right) .
$$

In order to verify that $\rho_{\beta \alpha}(\zeta)$ is independent of the choices made on $\xi_{a, \alpha}, \eta, \xi_{b, \beta}$, suppose we have two different choices $\left(\xi_{a, \alpha}, \eta, \xi_{b, \beta}\right)$ and $\left(\xi_{a^{\prime}, \alpha}, \eta^{\prime}, \xi_{b^{\prime}, \beta}\right)$. The fact that Domain $\left(\phi_{\zeta}\right)$ and Range $\left(\phi_{\zeta}\right)$ are connected allows us to reduce the problem to the following special cases: (1) $\xi_{a, \alpha}=\xi_{a^{\prime}, \alpha}$ and Domain $\left(\phi_{\eta}\right) \cap$ Domain $\left(\phi_{\eta^{\prime}}\right) \neq \emptyset,(2) \xi_{b, \beta}=\xi_{b^{\prime}, \beta}$ and Range $\left(\phi_{\eta}\right) \cap$ Range $\left(\phi_{\eta^{\prime}}\right) \neq \emptyset$. We shall only consider the first case, the second case is completely parallel. Under the assumption that Domain $\left(\phi_{\eta}\right) \cap$ Domain $\left(\phi_{\eta^{\prime}}\right) \neq \emptyset$, there is an $\epsilon \in T\left(U_{b}, U_{b^{\prime}}\right)$ such that $\eta^{\prime}=\epsilon \circ \eta(\mathbf{a})$ for some $\mathbf{a} \in \Lambda(\eta, \epsilon)$. It follows from the fact $\xi_{b, \beta} \circ \eta \circ \xi_{a, \alpha}^{-1}=\zeta=$ $\xi_{b^{\prime}, \beta} \circ \eta^{\prime} \circ \xi_{a^{\prime}, \alpha}^{-1}$ and the assumption that $\xi_{a, \alpha}=\xi_{a^{\prime}, \alpha}$ that $\xi_{b, \beta}=\xi_{b^{\prime}, \beta} \circ \epsilon\left(\phi_{\eta}(\mathbf{a})\right)$, which in turn implies that $\rho_{b^{\prime} b}(\epsilon)=\left(\xi_{b^{\prime}, \beta}^{\prime}\right)^{-1} \circ \xi_{b, \beta}^{\prime}(x), \forall x \in f_{b}\left(\phi_{\eta}(\mathbf{a})\right)$, by (3.1.9). With this relation, $\xi_{b, \beta}^{\prime} \circ \rho_{b a}(\eta) \circ\left(\xi_{a, \alpha}^{\prime}\right)^{-1}\left(\phi_{\xi_{a, \alpha}^{\prime}}\left(f_{a}(x)\right)\right)=\xi_{b^{\prime}, \beta}^{\prime} \circ \rho_{b^{\prime} a^{\prime}}\left(\eta^{\prime}\right) \circ\left(\xi_{a^{\prime}, \alpha}^{\prime}\right)^{-1}\left(\phi_{\xi_{a^{\prime}, \alpha}^{\prime}}\left(f_{a^{\prime}}(x)\right)\right), \forall x \in \mathbf{a}$, follows by recalling $\rho_{b^{\prime} a^{\prime}}\left(\eta^{\prime}\right)=\rho_{b^{\prime} b}(\epsilon) \circ \rho_{b a}(\eta)\left(\left\{f_{a}\right\}(\mathbf{a})\right)$. Hence $\rho_{\beta \alpha}(\zeta)$ is well-defined.

It remains to verify that $\left(\left\{f_{\alpha}\right\},\left\{\overline{\left.\left.\rho_{\beta \alpha}\right\}\right)}: \Gamma\left\{U_{\alpha}\right\} \rightarrow \Gamma\left\{U_{\alpha^{\prime}}^{\prime}\right\}\right.\right.$ is a homomorphism whose equivalence class is $\Phi$, and $\rho_{\alpha \alpha}=\rho_{\alpha}$ for each $\alpha$. The latter follows easily by comparing (3.1.9) with (3.1.10). In order to show that $\left(\left\{f_{\alpha}\right\},\left\{\rho_{\beta \alpha}\right\}\right)$ is a homomorphism, we need to check (a) $f_{\beta} \circ \phi_{\zeta}=\phi_{\rho_{\beta \alpha}(\zeta)} \circ f_{\alpha}, \forall \zeta \in T\left(U_{\alpha}, U_{\beta}\right)$, (b) $\rho_{\gamma \alpha}(\delta \circ \zeta(\mathbf{a}))=\rho_{\gamma \beta}(\delta) \circ \rho_{\beta \alpha}(\zeta)\left(\left\{f_{\alpha}\right\}(\mathbf{a})\right), \forall \mathbf{a} \in$ $\Lambda(\zeta, \delta), \zeta \in T\left(U_{\alpha}, U_{\beta}\right), \delta \in T\left(U_{\beta}, U_{\gamma}\right)$. The former follows directly from (3.1.10) and (3.1.8). For the latter, we first observe that we can choose $a \in I_{\alpha}, b \in I_{\beta}, c \in I_{\gamma}$ such that $\zeta=$ $\xi_{b, \beta} \circ \eta \circ \xi_{a, \alpha}^{-1}, \delta=\xi_{c, \gamma} \circ \epsilon \circ \xi_{b, \beta}^{-1}$ for some $\xi_{a, \alpha} \in T\left(U_{a}, U_{\alpha}\right), \xi_{b, \beta} \in T\left(U_{b}, U_{\beta}\right), \xi_{c, \gamma} \in T\left(U_{c}, U_{\gamma}\right)$ and some $\eta \in T\left(U_{a}, U_{b}\right), \epsilon \in T\left(U_{b}, U_{c}\right)$ satisfying Range $\left(\phi_{\eta}\right) \cap$ Domain $\left(\phi_{\epsilon}\right) \neq \emptyset$. Moreover, there is an element $\mathbf{b} \in \Lambda(\eta, \epsilon)$ contained in $\phi_{\xi_{a, \alpha}}^{-1}(\mathbf{a})$ such that $\delta \circ \zeta(\mathbf{a})=\xi_{c, \gamma} \circ(\epsilon \circ \eta(\mathbf{b})) \circ \xi_{a, \alpha}^{-1}$. With this relation, one can easily deduce (b) from (3.1.10) and the equations $\rho_{c a}(\epsilon \circ \eta(\mathbf{b}))=$ $\rho_{c b}(\epsilon) \circ \rho_{b a}(\eta)\left(\underline{\left\{f_{a}\right\}}(\mathbf{b})\right)$. Finally, it is straightforward from (3.1.8), (3.1.9) and (3.1.10) that 
$\tau=\left(\left\{f_{a}\right\},\left\{\rho_{b a}\right\}\right)$ is induced by $\left(\left\{f_{\alpha}\right\},\left\{\rho_{\beta \alpha}\right\}\right)$, hence the equivalence class of the latter is also the given map $\Phi$.

\subsection{Proof OF TheOREM 1.3}

First of all, we give the precise definition of the assumption on the domain orbispace $X$ in Theorem 1.3. An orbispace $X$ is said to be paracompact, locally compact and Hausdorff provided that the following conditions are satisfied:

- The underlying space $X$ is paracompact, locally compact, and Hausdorff.

- Each local chart $\widehat{U}_{i}$ of $X$ is locally compact and $\pi_{U_{i}}: \widehat{U}_{i} \rightarrow X$ is proper.

- Each local chart $\widehat{U}_{i}$ of $X$ is Hausdorff.

These conditions will be imposed on $X$ throughout this subsection. Finally, we shall fix the notation $\left[X ; X^{\prime}\right]$ for the set of maps of orbispaces from $X$ to $X^{\prime}$.

Lemma 3.2.1 Any open cover of $X$ has a refinement $\left\{U_{\alpha} \mid \alpha \in \Lambda\right\}$, which is locally finite and the closure $\overline{U_{\alpha}}$ is compact. Furthermore, there is an open cover $\left\{V_{\alpha} \mid \alpha \in \Lambda\right\}$ such that $\overline{V_{\alpha}} \subset U_{\alpha}$ for all $\alpha \in \Lambda$.

Proof Let $\left\{U_{i}\right\}$ be any given open cover. Since the underlying space $X$ is locally compact and Hausdorff, there is a refinement $\left\{U_{a}\right\}$ where each $U_{a}$ has a compact closure. On the other hand, the underlying space $X$ is paracompact, so $\left\{U_{a}\right\}$ has a locally finite refinement $\left\{U_{\alpha} \mid \alpha \in \Lambda\right\}$. It is easy to see that $\left\{U_{\alpha} \mid \alpha \in \Lambda\right\}$ is a desired refinement of $\left\{U_{i}\right\}$.

We construct $\left\{V_{\alpha}\right\}$ as follows. Let $S$ be the set of the subsets $I$ of $\Lambda$ satisfying the following condition: for each $\alpha \in I$, there exists a $V_{\alpha}$ such that $\overline{V_{\alpha}} \subset U_{\alpha}$ and $X=\cup_{\alpha \in I} V_{\alpha} \cup_{\alpha \in \Lambda \backslash I} U_{\alpha}$. We shall prove that $\Lambda \in S$.

First of all, $S$ is nonempty. To see this, we pick an $\alpha_{0} \in \Lambda$ and set $W_{\alpha_{0}}=X \backslash \cup_{\alpha \neq \alpha_{0}} U_{\alpha}$, which is a compact subset of $U_{\alpha_{0}}$. Since the underlying space $X$ is Hausdorff, and $\overline{U_{\alpha_{0}}} \backslash U_{\alpha_{0}}$ is compact, we see that for any $p \in W_{\alpha_{0}}$, there is a neighborhood $O_{p}$ of $p$ with $\overline{O_{p}} \subset U_{\alpha_{0}}$. The compactness of $W_{\alpha_{0}}$ implies that there are finitely many $p_{1}, \cdots, p_{n} \in W_{\alpha_{0}}$ such that $W_{\alpha_{0}} \subset \cup_{i=1}^{n} O_{p_{i}}$. We take $V_{\alpha_{0}}=\cup_{i=1}^{n} O_{p_{i}}$. Then clearly $\overline{V_{\alpha_{0}}} \subset U_{\alpha_{0}}$ and $X=\cup_{\alpha \neq \alpha_{0}} U_{\alpha} \cup V_{\alpha_{0}}$. Hence $\left\{\alpha_{0}\right\} \in S$.

Secondly, $S$ is partially ordered: $I \leq I^{\prime}$ iff $I \subset I^{\prime}$. Let $T \subset S$ be a linearly ordered subset. We shall prove that $I(T)=\cup_{I \in T} I \in S$, or equivalently, $X=\cup_{\alpha \in I(T)} V_{\alpha} \cup_{\alpha \in \Lambda \backslash I(T)} U_{\alpha}$. Given any $p \in X$, since $\left\{U_{\alpha} \mid \alpha \in \Lambda\right\}$ is locally finite, there are only finitely many elements, say $U_{\alpha_{1}}, \cdots, U_{\alpha_{k}}$, which contains $p$. We need to show that if $\alpha_{i} \in I(T)$ for all $1 \leq i \leq k$, then $p \in \cup_{\alpha \in I(T)} V_{\alpha}$. To this end, we observe that since $T$ is linearly ordered, there exists an $I \in T$ containing all $\alpha_{i}, 1 \leq i \leq k$. Then $p \in X=\cup_{\alpha \in I} V_{\alpha} \cup_{\alpha \in \Lambda \backslash I} U_{\alpha}$ implies that $p \in \cup_{\alpha \in I} V_{\alpha} \subset \cup_{\alpha \in I(T)} V_{\alpha}$. Hence $I(T) \in S$.

Finally, we apply Zorn's Lemma to $S$ to conclude that there is a maximal element $I_{0}$ in $S$. Then a verbatim proof of $S \neq \emptyset$ shows that $I_{0}=\Lambda$. Hence $\Lambda \in S$. 
We need to consider a class of special local charts $\left\{U_{i}\right\}$ on $X$, which are dictated by the following conditions: (1) the closure $\overline{U_{i}}$ is a compact subset, (2) there is a space $\widehat{\widehat{U_{i}}}$ containing $\widehat{U}_{i}$ as its interior, with an action of $G_{U_{i}}$ extending the action of $G_{U_{i}}$ on $\widehat{U}_{i}$, and a map $\pi_{\overline{U_{i}}}: \widehat{\bar{U}_{i}} \rightarrow X$ extending $\pi_{U_{i}}$, such that $\widehat{\bar{U}_{i}} / G_{U_{i}}$ is homeomorphic to $\overline{U_{i}}$ under $\pi_{\overline{U_{i}}}$, and (3) for any pair of such $U_{i}, U_{j}$ with $U_{i} \cap U_{j} \neq \emptyset$, the homeomorphism $\phi_{\xi}$ associated to any $\xi \in T\left(U_{i}, U_{j}\right)$ can be extended to a homeomorphism between the closures of its domain and range. We call this class of local charts 'admissible'.

In light of Lemma 3.2.1, we may assume without loss of generality for the remaining subsections that in any homomorphisms $\left(\left\{f_{\alpha}\right\},\left\{\rho_{\beta \alpha}\right\}\right): \Gamma\left\{U_{\alpha}\right\} \rightarrow \Gamma\left\{U_{\alpha^{\prime}}^{\prime}\right\}$ under consideration, the following conditions are satisfied: (1) the cover $\left\{U_{\alpha}\right\}$ is locally finite and each local chart $U_{\alpha}$ is admissible, (2) each $f_{\alpha}$ can be extended over (uniquely) to the compact closure $\widehat{U_{\alpha}}$. We call this class of homomorphisms 'admissible'.

Let $\left(\left\{f_{\alpha}^{(0)}\right\},\left\{\rho_{\beta \alpha}\right\}\right): \Gamma\left\{U_{\alpha}\right\} \rightarrow \Gamma\left\{U_{\alpha^{\prime}}^{\prime}\right\}$ be any admissible homomorphism. We define

$$
\mathcal{O}_{\left\{\rho_{\beta \alpha}\right\}}=\left\{\sigma \mid \sigma=\left(\left\{f_{\alpha}\right\},\left\{\rho_{\beta \alpha}\right\}\right): \Gamma\left\{U_{\alpha}\right\} \rightarrow \Gamma\left\{U_{\alpha^{\prime}}^{\prime}\right\} \text { is admissible }\right\}
$$

and

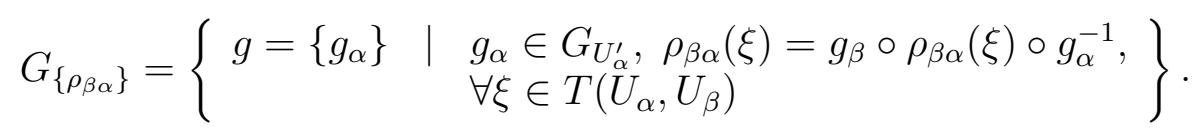

Note that $G_{\left\{\rho_{\beta \alpha}\right\}}$ is a group under $\left\{g_{\alpha}\right\}\left\{h_{\alpha}\right\}=\left\{g_{\alpha} h_{\alpha}\right\}$. We give a topology on $\mathcal{O}_{\left\{\rho_{\beta \alpha}\right\}}$ as follows. For each index $\alpha$, let $\underline{K_{\alpha}}$ be a finite set of compact subsets of $\widehat{U_{\alpha}}$, denoted by $\left\{K_{\alpha, i} \mid i \in I_{\alpha}\right\}$, and let $\underline{O_{\alpha}}$ be a finite set of open subsets of $\widehat{U_{\alpha}^{\prime}}$, denoted by $\left\{O_{\alpha, i} \mid i \in I_{\alpha}\right\}$. We define

$$
\mathcal{O}_{\left\{\rho_{\beta \alpha}\right\}}\left(\left\{\underline{K_{\alpha}}\right\},\left\{\underline{O_{\alpha}}\right\}\right)=\left\{\left\{f_{\alpha}\right\} \in \mathcal{O}_{\left\{\rho_{\beta \alpha}\right\}} \mid f_{\alpha}\left(K_{\alpha, i}\right) \subset O_{\alpha, i}, \forall i \in I_{\alpha}\right\} .
$$

Note that $\bigcap_{j} \mathcal{O}_{\left\{\rho_{\beta \alpha}\right\}}\left(\left\{K_{\alpha}^{(j)}\right\},\left\{O_{\alpha}^{(j)}\right\}\right)=\mathcal{O}_{\left\{\rho_{\beta \alpha}\right\}}\left(\left\{\bigcup_{j} K_{\alpha}^{(j)}\right\},\left\{\bigcup_{j} O_{\alpha}^{(j)}\right\}\right)$. Hence the set of (3.2.3) forms a base of a topology on $\overline{\mathcal{O}}_{\left\{\rho_{\beta \alpha}\right\}}$. The group $\overline{G_{\left\{\rho_{\beta \alpha}\right\}}}$ acts on $\mathcal{O}_{\left\{\rho_{\beta \alpha}\right\}}$ continuously by the formula $\left\{g_{\alpha}\right\} \cdot\left\{f_{\alpha}\right\}=\left\{g_{\alpha} \circ f_{\alpha}\right\}$, whose orbit consists of mutually conjugate homomorphisms. We denote the space of orbits by $\left[\mathcal{O}_{\left\{\rho_{\beta \alpha}\right\}}\right]$, which is given with the quotient topology. By Lemma 3.1.2, each $\left[\mathcal{O}_{\left\{\rho_{\beta \alpha}\right\}}\right]$ can be naturally regarded as a subset of $\left[X ; X^{\prime}\right]$. The image of $\mathcal{O}_{\left\{\rho_{\beta \alpha}\right\}}\left(\left\{\underline{\left.K_{\alpha}\right\}},\left\{\underline{O_{\alpha}}\right\}\right)\right.$ in $\left[\mathcal{O}_{\left\{\rho_{\beta \alpha}\right\}}\right]$ is denoted by $\left[\mathcal{O}_{\left\{\rho_{\beta \alpha}\right\}}\left(\left\{\underline{K_{\alpha}}\right\},\left\{\underline{O_{\alpha}}\right\}\right)\right]$. Note that the set of all $\left.\left[\mathcal{O}_{\left\{\rho_{\beta \alpha}\right\}}\left(\left\{\underline{K_{\alpha}}\right\}, \underline{\left\{O_{\alpha}\right.}\right\}\right)\right]$ forms a base of the quotient topology on $\left[\mathcal{O}_{\left\{\rho_{\beta \alpha}\right\}}\right]$.

Lemma 3.2.2 Let $\tau \in \mathcal{O}_{\left\{\rho_{b a}\right\}}$ and $\sigma \in \mathcal{O}_{\left\{\rho_{\beta \alpha}\right\}}$ be any pair of equivalent homomorphisms.

- To any representative $\bar{\gamma}$ of an element $\gamma \in \Gamma_{\sigma \tau}$, there is associated a local homeomorphism $\phi_{\bar{\gamma}}$ from an open neighborhood of $\tau$ in $\mathcal{O}_{\left\{\rho_{b a}\right\}}$ onto an open neighborhood of $\sigma$ in $\mathcal{O}_{\left\{\rho_{\beta \alpha}\right\}}$ such that $\phi_{\bar{\gamma}}(\tau)=\sigma$.

- The germ of $\phi_{\bar{\gamma}}$ at $\tau$, denoted by $\phi_{\gamma}$, depends only on the equivalence class $\gamma \in \Gamma_{\sigma \tau}$, and for any $\gamma \in \Gamma_{\sigma \tau}$ and $\gamma^{\prime} \in \Gamma_{\tau \kappa}$,

$$
\phi_{\gamma} \circ \phi_{\gamma^{\prime}}=\phi_{\gamma \circ \gamma^{\prime}}
$$

Moreover, if $\sigma=\phi_{\bar{\gamma}}(\tau)$ is also in $\mathcal{O}_{\left\{\rho_{b a}\right\}}$, then $\Gamma_{\sigma \tau} \subset G_{\left\{\rho_{b a}\right\}}$ and $\phi_{\gamma}$ is induced by the action of $G_{\left\{\rho_{b a}\right\}}$ on $\mathcal{O}_{\left\{\rho_{b a}\right\}}$. 
- For any $\tau^{\prime} \in$ Domain $\left(\phi_{\bar{\gamma}}\right), \sigma^{\prime}=\phi_{\bar{\gamma}}\left(\tau^{\prime}\right)$ is equivalent to $\tau^{\prime}$, and $\gamma \in \Gamma_{\sigma \tau}$ is canonically associated with a $\gamma^{\prime} \in \Gamma_{\sigma^{\prime} \tau^{\prime}}$ such that the germ of $\phi_{\bar{\gamma}}$ at $\tau^{\prime}$ equals $\phi_{\gamma^{\prime}}$.

The proof of Lemma 3.2.2 is postponed to the end of this subsection.

\section{Proof of Theorem 1.3:}

We give $\left[X ; X^{\prime}\right]$ a topology which is generated by the set of $\left[\mathcal{O}_{\left\{\rho_{\beta \alpha}\right\}}\left(\left\{\underline{K_{\alpha}}\right\},\left\{\underline{O_{\alpha}}\right\}\right)\right]$ for all possible data $\left\{\rho_{\beta \alpha}\right\},\left\{\underline{K_{\alpha}}\right\}$, and $\left\{\underline{O_{\alpha}}\right\}$. By the existence of local homeomorphisms $\phi_{\bar{\gamma}}$, each $\left[\mathcal{O}_{\left\{\rho_{\beta \alpha}\right\}}\right]$ with the quotient topology is an open subset of $\left[X ; X^{\prime}\right]$. Thus we obtained an open cover $\left\{\left[\mathcal{O}_{\left\{\rho_{\beta \alpha}\right\}}\right]\right\}$ of $\left[X ; X^{\prime}\right]$, where for each $\left[\mathcal{O}_{\left\{\rho_{\beta \alpha}\right\}}\right]$, there is a space $\mathcal{O}_{\left\{\rho_{\beta \alpha}\right\}}$ and a discrete group $G_{\left\{\rho_{\beta \alpha}\right\}}$ acting continuously on $\mathcal{O}_{\left\{\rho_{\beta \alpha}\right\}}$ such that $\left[\mathcal{O}_{\left\{\rho_{\beta \alpha}\right\}}\right]=\mathcal{O}_{\left\{\rho_{\beta \alpha}\right\}} / G_{\left\{\rho_{\beta \alpha}\right\}}$.

Consider the groupoid $\Gamma=\bigcup_{(\sigma, \tau)} \Gamma_{\sigma \tau}$, where $\sigma, \tau$ are running over the set of all admissible homomorphisms. The disjoint union $U=\sqcup \mathcal{O}_{\left\{\rho_{\beta \alpha}\right\}}$ is embedded into $\Gamma$ as the space of units. The mappings $\alpha, \omega: \Gamma \rightarrow U$ are given by $\alpha(\gamma)=\tau, \omega(\gamma)=\sigma$ for any $\gamma \in \Gamma_{\sigma \tau}$. Clearly the restriction of $\Gamma$ to each $\mathcal{O}_{\left\{\rho_{\beta \alpha}\right\}}$ is the product groupoid $G_{\left\{\rho_{\beta \alpha}\right\}} \times \mathcal{O}_{\left\{\rho_{\beta \alpha}\right\}}$ (cf. Lemma 3.1.2), and the space of $\Gamma$-orbits is isomorphic to $\left[X ; X^{\prime}\right]$. It remains to put a topology on $\Gamma$ so that it becomes an étale topological groupoid, such that the induced topology on $U=\sqcup \mathcal{O}_{\left\{\rho_{\beta \alpha}\right\}}$ is defined by (3.2.3). By Lemma 3.2.2, $\left\{\phi_{\bar{\gamma}}\right\}$ generates a pseudogroup acting on $U=\bigsqcup \mathcal{O}_{\left\{\rho_{\beta \alpha}\right\}}$ by local homeomorphisms, where $U$ is given the topology defined by (3.2.3), and the corresponding space of germs of the elements in the pseudogroup is naturally identified with $\Gamma$. Thus $\Gamma$, when equipped with the étale topology, is an étale topological groupoid under which $\left[X ; X^{\prime}\right]$ canonically becomes an orbispace.

Proposition 3.2.3 Let $X, Y$ be paracompact, locally compact and Hausdorff, and $Z$ be any orbispace. Then the mapping $(\Phi, \Psi) \mapsto \Psi \circ \Phi, \forall \Phi \in[X ; Y], \Psi \in[Y ; Z]$, defines a continuous map between the underlying spaces of $[X ; Y] \times[Y ; Z]$ and $[X ; Z]$.

Proof We may represent $\Phi, \Psi$ by admissible homomorphisms $\sigma=\left(\left\{f_{\alpha}\right\},\left\{\rho_{\beta \alpha}\right\}\right), \tau=$ $\left(\left\{g_{a}\right\},\left\{\eta_{b a}\right\}\right)$, such that $\Psi \circ \Phi$ is represented by $\sigma \circ \tau=\left(\left\{h_{a}\right\},\left\{\delta_{b a}\right\}\right)$, which is also admissible. Here $h_{a}=f_{\theta(a)} \circ g_{a}, \delta_{b a}=\rho_{\theta(b) \theta(a)} \circ \eta_{b a}$ for some mapping of indices $\theta: a \mapsto \alpha$ (cf. proof of Theorem 2.2.7). Let $\mathcal{O}_{\left\{\delta_{b a}\right\}}\left(\left\{\underline{K_{a}}\right\},\left\{\underline{O_{a}}\right\}\right)$ be any given neighborhood of $\sigma \circ \tau$, where $\underline{K_{a}}=\left\{K_{a, i} \mid i \in I_{a}\right\}$ and $\underline{O_{a}}=\left\{O_{a, i} \mid i \in I_{a}\right\}$. We set $\underline{O_{\alpha}}=\emptyset$ if $\alpha \neq \theta(a)$ for any index $a$, and set $\underline{O_{\alpha}}=\left\{O_{a, i} \mid i \in I_{a}\right\}$ if $\alpha=\theta(a)$ for some index $a$. Since $Y$ is locally compact and Hausdorff, we may choose $Q_{a, i}, i \in I_{a}$, such that $g_{a}\left(K_{a, i}\right) \subset Q_{a, i}$, and the closure $\overline{Q_{a, i}}=L_{a, i}$ is compact, satisfying $f_{\theta(a)}\left(L_{a, i}\right) \subset O_{a, i}$. Now set $L_{\alpha}=\emptyset$ if $\alpha \neq \theta(a)$ for any index $a, \underline{L_{\alpha}}=\left\{L_{a, i} \mid i \in I_{a}\right\}$ if $\alpha=\theta(a)$ for some index $a$, and $\bar{Q}_{a}=\left\{Q_{a, i} \mid i \in I_{a}\right\}$. Then $\mathcal{O}_{\left\{\rho_{\beta \alpha}\right\}}\left(\left\{\underline{L_{\alpha}}\right\},\left\{\underline{O_{\alpha}}\right\}\right)$ and $\mathcal{O}_{\left\{\eta_{b a}\right\}}\left(\left\{\underline{K_{a}}\right\},\left\{\underline{Q_{a}}\right\}\right)$ are neighborhoods of $\sigma$ and $\tau$ respectively, such that for any $\sigma^{\prime} \in \mathcal{O}_{\left\{\rho_{\beta \alpha}\right\}}\left(\left\{\underline{L_{\alpha}}\right\},\left\{\underline{O_{\alpha}}\right\}\right), \overline{\tau^{\prime}} \in \mathcal{O}_{\left\{\eta_{b a}\right\}}\left(\left\{\underline{K_{a}}\right\},\left\{\underline{Q_{a}}\right\}\right), \sigma^{\prime} \circ \tau^{\prime}$ is defined and lies in $\mathcal{O}_{\left\{\delta_{b a}\right\}}\left(\left\{\underline{K_{a}}\right\},\left\{\underline{O_{a}}\right\}\right)$. Thus the mapping $(\Phi, \Psi) \mapsto \Psi \circ \Phi$ is continuous.

\section{Proof of Lemma 3.2.2}

Let $\tau=\left\{f_{a}\right\}, \sigma=\left\{f_{\alpha}\right\}$. We shall first construct the local homeomorphism $\phi_{\bar{\gamma}}$ for the special circumstance where $\tau$ is induced by $\sigma$, and the following condition

$$
U_{a}^{\prime} \subset U_{\alpha}^{\prime} \text { whenever } U_{a} \subset U_{\alpha}
$$


is satisfied. Note that (3.2.5) can be always arranged. This is because $\left\{U_{\alpha}\right\}$ is locally finite, therefore for each $U_{a}$, there are only finitely many $U_{\alpha}$ 's containing $U_{a}$, so that one may replace $U_{a}^{\prime}$ by a smaller one $U_{a}^{\prime} \cap U_{\alpha}^{\prime}$ whenever $U_{a} \subset U_{\alpha}$ occurs, and then work with the homomorphism induced by $\tau$.

On the other hand, in light of Lemma 3.2.1, we may assume, by passing to an induced homomorphism of $\tau$, that for each index $a$, there is an $\alpha$ such that $\overline{U_{a}} \subset U_{\alpha}$. Moreover, there exists an open cover $\left\{V_{a}\right\}$ of $X$, which will be fixed throughout, such that $\overline{V_{a}} \subset U_{a}$. We set $K_{a}=\pi_{U_{a}}^{-1}\left(\overline{V_{a}}\right)$.

Now given any representative $\bar{\gamma}=\left(\theta,\left\{\xi_{a}\right\},\left\{\xi_{a}^{\prime}\right\}\right)$ of $\gamma \in \Gamma_{\sigma \tau}$, where $\theta: a \mapsto \alpha$ is a mapping of indexes such that

$$
\overline{U_{a}} \subset U_{\theta(a)}, \forall a,
$$

and $\xi_{a} \in T\left(U_{a}, U_{\theta(a)}\right), \xi_{a}^{\prime} \in T\left(U_{a}^{\prime}, U_{\theta(a)}^{\prime}\right)$, with

$$
f_{a}=\left(\phi_{\xi_{a}^{\prime}}\right)^{-1} \circ f_{\theta(a)} \circ \phi_{\xi_{a}}, \forall a,
$$

and for any $\eta \in T\left(U_{a}, U_{b}\right)$,

$$
\rho_{b a}(\eta)=\left(\xi_{b}^{\prime}\right)^{-1} \circ \rho_{\theta(b) \theta(a)}\left(\xi_{b} \circ \eta \circ \xi_{a}^{-1}\right) \circ \xi_{a}^{\prime}(x) \forall x \in f_{a}\left(\text { Domain }\left(\phi_{\eta}\right)\right),
$$

we shall construct an open embedding $\phi_{\bar{\gamma}}$ from an open neighborhood $\mathcal{O}_{\left\{\rho_{b a}\right\}}\left(\left\{\underline{\left.L_{a}^{0}\right\}},\left\{\underline{\left.Q_{a}^{0}\right\}}\right.\right.\right.$ ) of

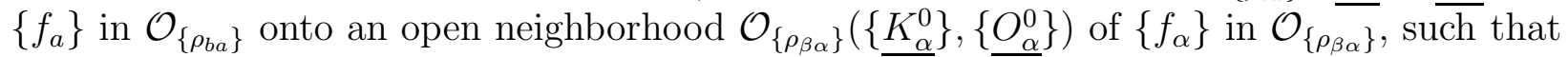
$\phi_{\bar{\gamma}}\left(\left\{f_{a}\right\}\right)=\left\{f_{\alpha}\right\}$, and for any $\left\{f_{a}^{\prime}\right\} \in \mathcal{O}_{\left\{\rho_{b a}\right\}}\left(\left\{\underline{L_{a}^{0}}\right\},\left\{\underline{\left.Q_{a}^{0}\right\}}\right),\left\{\overline{\left.f_{a}^{\prime}\right\}}\right.\right.$ is induced by $\left\{f_{\alpha}^{\prime}\right\}=\phi_{\bar{\gamma}}\left(\left\{f_{a}^{\prime}\right\}\right)$ via the data $\bar{\gamma}=\left(\theta,\left\{\xi_{a}\right\},\left\{\xi_{a}^{\prime}\right\}\right)$.

We begin by defining a mapping from $T\left(U_{a}, U_{\alpha}\right)$ to $T\left(U_{a}^{\prime}, U_{\alpha}^{\prime}\right)$ for each pair $(a, \alpha)$ with $U_{a} \cap U_{\alpha} \neq \emptyset$, which is denoted by $\xi \mapsto \xi^{\prime}$, by the formula

$$
\xi^{\prime}=\rho_{\alpha \theta(a)}\left(\xi \circ \xi_{a}^{-1}\right) \circ \xi_{a}^{\prime}(x), \forall x \in f_{a}\left(\text { Domain }\left(\phi_{\xi}\right)\right) .
$$

Note that $\xi_{a} \mapsto \xi_{a}^{\prime}, \forall a$, under $\xi \mapsto \xi^{\prime}$ defined by (3.2.8). Moreover, since $U_{a}^{\prime} \subset U_{\theta(a)}^{\prime}$ by (3.2.5), one has

$$
\text { Domain }\left(\phi_{\xi^{\prime}}\right) \subset \text { Domain }\left(\phi_{\rho_{\alpha \theta(a)}\left(\xi_{\circ} \xi_{a}^{-1}\right)} \circ \phi_{\xi_{a}^{\prime}}\right) \text {. }
$$

We determine the data $\left\{\underline{L_{a}^{0}}\right\},\left\{\underline{Q_{a}^{0}}\right\}$ as follows. Let index a be given. (1) Suppose $U_{a} \cap U_{\alpha} \neq$ $\emptyset$ for some index $\alpha$. Since $f_{a}\left(\overline{\pi_{U_{a}}^{-1}\left(U_{a} \cap U_{\alpha}\right)}\right) \subset \widehat{U_{a}^{\prime}}$ is compact, there are only finitely many components $\left\{Q_{a, \alpha}^{i} \mid i \in \Lambda(\alpha)\right\}$ of $\pi_{U_{a}^{\prime}}^{-1}\left(U_{a}^{\prime} \cap U_{\alpha}^{\prime}\right)$ which contain $f_{a}\left(\overline{\pi_{U_{a}}^{-1}\left(U_{a} \cap U_{\alpha}\right)}\right)$. We let $L_{a, \alpha}^{i} \subset \widehat{\overline{U_{a}}}$ be the union of $\overline{\text { Domain }\left(\phi_{\xi}\right)}$ for all $\xi \in T\left(U_{a}, U_{\alpha}\right)$ such that Domain $\left(\phi_{\xi^{\prime}}\right)=Q_{a, \alpha}^{i}$. We set $Q_{a}^{(1)}=\left\{Q_{a, \alpha}^{i} \mid i \in \Lambda(\alpha), U_{a} \cap U_{\alpha} \neq \emptyset\right\}$, and set $L_{a}^{(1)}=\left\{L_{a, \alpha}^{i} \cap K_{a} \mid i \in \Lambda(\alpha), U_{a} \cap U_{\alpha} \neq\right.$

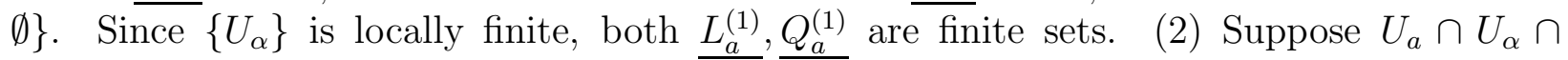
$U_{b} \neq \emptyset$ for some indexes $\alpha, b$. Since $f_{\theta(a)}\left(\overline{\pi_{U_{\theta(a)}}^{-1}\left(U_{\theta(a)} \cap U_{\alpha} \cap U_{\theta(b)}\right)}\right)$ is a compact subset in $\pi_{U_{\theta(a)}^{\prime}}^{-1}\left(U_{\theta(a)}^{\prime} \cap U_{\alpha}^{\prime} \cap U_{\theta(b)}^{\prime}\right)$, it is contained in finitely many components $\left\{W_{a,(\alpha, b)}^{i} \mid i \in \Lambda(\alpha, b)\right\}$. We set $Q_{a,(\alpha, b)}^{i}=\left(\phi_{\xi_{a}^{\prime}}\right)^{-1}\left(W_{a,(\alpha, b)}^{i}\right), i \in \Lambda(\alpha, b)$, and let $L_{a,(\alpha, b)}^{i}$ be the union of the closure of $\mathbf{a} \in \Lambda\left(\zeta_{a}, \zeta_{b}^{-1}\right)$ such that $f_{\theta(a)} \circ \phi_{\xi_{a}}(\mathbf{a}) \subset W_{a,(\alpha, b)}^{i}$, where $\zeta_{a} \in T\left(U_{a}, U_{\alpha}\right), \zeta_{b} \in T\left(U_{b}, U_{\alpha}\right)$. We define $\underline{Q_{a}^{(2)}}=\left\{Q_{a,(\alpha, b)}^{i} \mid i \in \Lambda(\alpha, b), U_{a} \cap U_{\alpha} \cap U_{b} \neq \emptyset\right\}, \underline{L_{a}^{(2)}}=\left\{L_{a,(\alpha, b)}^{i} \cap K_{a} \mid i \in \Lambda(\alpha, b), U_{a} \cap\right.$ $U_{\alpha} \cap \overline{U_{b} \neq \emptyset}$. Both $\underline{L_{a}^{(2)}}, \underline{Q_{a}^{(2)}}$ are finite sets because $\left\{\overline{\left.U_{\alpha}\right\},\{} U_{b}\right\}$ are locally finite. (3) Suppose 
$U_{a} \cap U_{\alpha} \cap U_{\beta} \neq \emptyset$ for some indexes $\alpha, \beta$. Since $f_{\theta(a)}\left(\overline{\pi_{U_{\theta(a)}}^{-1}\left(U_{\theta(a)} \cap U_{\alpha} \cap U_{\beta}\right)}\right)$ is a compact subset in $\pi_{U_{\theta(a)}^{\prime}}^{-1}\left(U_{\theta(a)}^{\prime} \cap U_{\alpha}^{\prime} \cap U_{\beta}^{\prime}\right)$, it is contained in finitely many components $\left\{W_{a,(\alpha, \beta)}^{i} \mid i \in \Lambda(\alpha, \beta)\right\}$. We set $Q_{a,(\alpha, \beta)}^{i}=\left(\phi_{\xi_{a}^{\prime}}\right)^{-1}\left(W_{a,(\alpha, \beta)}^{i}\right), i \in \Lambda(\alpha, \beta)$, and let $L_{a,(\alpha, \beta)}^{i}$ be the union of the closure of $\mathbf{a} \in \Lambda(\xi, \eta)$ such that $f_{\theta(a)} \circ \phi_{\xi_{a}}(\mathbf{a}) \subset W_{a,(\alpha, \beta)}^{i}$, where $\xi \in T\left(U_{a}, U_{\alpha}\right), \eta \in T\left(U_{\alpha}, U_{\beta}\right)$. We define $\underline{Q_{a}^{(3)}}=\left\{Q_{a,(\alpha, \beta)}^{i} \mid i \in \Lambda(\alpha, \beta), U_{a} \cap U_{\alpha} \cap U_{\beta} \neq \emptyset\right\}, \underline{L_{a}^{(3)}}=\left\{L_{a,(\alpha, \beta)}^{i} \cap K_{a} \mid i \in\right.$ $\left.\Lambda(\alpha, \beta), U_{a} \overline{\cap U}_{\alpha} \cap U_{\beta} \neq \emptyset\right\}$. Both $\underline{L_{a}^{(3)}}, \underline{Q_{a}^{(3)}}$ are finite sets because $\left\{U_{\alpha}\right\}$ is locally finite. (4) Suppose $U_{a} \cap U_{\alpha} \cap U_{\beta} \cap U_{\gamma} \neq \emptyset$ for some indexes $\alpha, \beta, \gamma$. Since $f_{a}\left(\overline{\pi_{U_{a}}^{-1}\left(U_{a} \cap U_{\alpha} \cap U_{\beta} \cap U_{\gamma}\right)}\right)$ is a compact subset in $\pi_{U_{a}^{\prime}}^{-1}\left(U_{a}^{\prime} \cap U_{\alpha}^{\prime} \cap U_{\beta}^{\prime} \cap U_{\gamma}^{\prime}\right)$, it is contained in finitely many components $\left\{Q_{a,(\alpha, \beta, \gamma)}^{i} \mid i \in I(\alpha, \beta, \gamma)\right\}$. We define $L_{a,(\alpha, \beta, \gamma)}^{i}=\left\{x \in \overline{\pi_{U_{a}}^{-1}\left(U_{a} \cap U_{\alpha} \cap U_{\beta} \cap U_{\gamma}\right)} \cap K_{a} \mid\right.$ $\left.f_{a}(x) \in Q_{a,(\alpha, \beta, \gamma)}^{i}\right\}$, and set $\frac{L_{a}^{(4)}}{=}\left\{L_{a,(\alpha, \beta, \gamma)}^{i} \mid i \in I(\alpha, \beta, \gamma), U_{a} \cap U_{\alpha} \cap U_{\beta} \cap U_{\gamma} \neq \emptyset\right\}$, $\underline{Q_{a}^{(4)}}=\left\{Q_{a,(\alpha, \beta, \gamma)}^{i} \mid i \in I(\alpha, \overline{\beta, \gamma}), U_{a} \cap U_{\alpha} \cap U_{\beta} \cap U_{\gamma} \neq \emptyset\right\}$. Then both $\underline{L_{a}^{(4)}}, \underline{Q_{a}^{(4)}}$ are finite $\overline{\text { sets }}$ because $\left\{U_{\alpha}\right\}$ is locally finite. We define $\underline{L_{a}^{0}}=\cup_{k=1}^{4} \underline{L_{a}^{(k)}}, \underline{Q_{a}^{0}}=\cup_{k=1}^{4} \underline{Q_{a}^{(k)}}$. Clearly $\left\{f_{a}\right\}$ is contained in $\mathcal{O}_{\left\{\rho_{b a}\right\}}\left(\left\{\underline{L_{a}^{0}}\right\},\left\{\underline{Q_{a}^{0}}\right\}\right)$.

We define the map $\phi_{\bar{\gamma}}: \mathcal{O}_{\left\{\rho_{b a}\right\}}\left(\left\{\underline{L_{a}^{0}}\right\},\left\{\underline{Q_{a}^{0}}\right\}\right) \rightarrow \mathcal{O}_{\left\{\rho_{\beta \alpha}\right\}}$. Given any $\left\{f_{a}^{\prime}\right\} \in \mathcal{O}_{\left\{\rho_{b a}\right\}}\left(\left\{\underline{L_{a}^{0}}\right\},\left\{\underline{Q_{a}^{0}}\right\}\right)$, $\left\{f_{\alpha}^{\prime}\right\}=\phi_{\bar{\gamma}}\left(\left\{f_{a}^{\prime}\right\}\right)$ is constructed as follows. For any $x \in \widehat{\overline{U_{\alpha}}}$, we pick a $U_{a}$ and a $\xi \in \bar{T}\left(U_{a}, \overline{U_{\alpha}}\right)$ such that $x \in \overline{\text { Range }\left(\phi_{\xi}\right)}$ and $\left(\phi_{\xi}\right)^{-1}(x) \in K_{a}$. Then by the assumption that $\left\{f_{a}^{\prime}\right\} \in$ $\mathcal{O}_{\left\{\rho_{b a}\right\}}\left(\left\{\underline{L_{a}^{0}}\right\},\left\{\underline{Q_{a}^{0}}\right\}\right)$, we have $f_{a}^{\prime} \circ\left(\phi_{\xi}\right)^{-1}(x) \subset$ Domain $\left(\phi_{\xi^{\prime}}\right)$. We define

$$
f_{\alpha}^{\prime}(x)=\phi_{\xi^{\prime}} \circ f_{a}^{\prime} \circ\left(\phi_{\xi}\right)^{-1}(x) .
$$

It remains to show that (1) each $f_{\alpha}^{\prime}$ is well-defined, (2) $\phi_{\rho_{\beta \alpha}(\eta)} \circ f_{\alpha}^{\prime}=f_{\beta}^{\prime} \circ \phi_{\eta}$ holds for any $\alpha, \beta$ and $\eta \in T\left(U_{\alpha}, U_{\beta}\right)$, (3) $\rho_{\gamma \alpha}(\eta \circ \zeta(\mathbf{a}))=\rho_{\gamma \beta}(\eta) \circ \rho_{\beta \alpha}(\zeta)\left(\left\{f_{\alpha}^{\prime}\right\}(\mathbf{a})\right)$ holds for any indexes $\alpha, \beta, \gamma$, and for any $\zeta \in T\left(U_{\alpha}, U_{\beta}\right), \eta \in T\left(U_{\beta}, U_{\gamma}\right)$, and $\mathbf{a} \in \overline{\Lambda(\zeta, \eta)}$, (4) $\left\{f_{a}^{\prime}\right\}$ is induced by $\left\{f_{\alpha}^{\prime}\right\}$ via the data $\bar{\gamma}=\left(\theta,\left\{\xi_{a}\right\},\left\{\xi_{a}^{\prime}\right\}\right)$, and $(5) \phi_{\bar{\gamma}}\left(\left\{f_{a}\right\}\right)=\left\{f_{\alpha}\right\}$.

The problem can be easily reduced to the following two types of identities. The first one is that for any $\xi \in T\left(U_{a}, U_{\alpha}\right)$,

$$
\phi_{\rho_{\alpha \theta(a)}\left(\xi \circ \xi_{a}^{-1}\right)} \circ\left(\phi_{\xi_{a}^{\prime}} \circ f_{a}^{\prime} \circ\left(\phi_{\xi_{a}}\right)^{-1}\right)=\left(\phi_{\xi^{\prime}} \circ f_{a}^{\prime} \circ\left(\phi_{\xi}\right)^{-1}\right) \circ \phi_{\xi \circ \xi_{a}^{-1}}
$$

on $\phi_{\xi_{a}}\left(K_{a} \cap\right.$ Domain $\left(\phi_{\xi}\right)$ ), which follows easily from (3.2.9). The second one is that for any $\xi \in T\left(U_{a}, U_{b}\right)$,

$$
\phi_{\rho_{\theta(b) \theta(a)}\left(\xi_{b} \circ \xi_{\circ} \xi_{a}^{-1}\right)} \circ\left(\phi_{\xi_{a}^{\prime}} \circ f_{a}^{\prime} \circ\left(\phi_{\xi_{a}}\right)^{-1}\right)=\left(\phi_{\xi_{b}^{\prime}} \circ f_{b}^{\prime} \circ\left(\phi_{\xi_{b}}\right)^{-1}\right) \circ \phi_{\xi_{b} \circ \xi^{\circ} \xi_{a}^{-1}}
$$

on $\phi_{\xi_{a}}$ (Domain $\left(\phi_{\xi}\right)$ ), which follows from the fact that $\phi_{\rho_{b a}(\xi)} \circ f_{a}^{\prime}=f_{b}^{\prime} \circ \phi_{\xi}$ and that Domain $\left(\phi_{\rho_{b a}(\xi)}\right)$ is contained in Domain $\left(\left(\phi_{\xi_{b}^{\prime}}\right)^{-1} \circ \phi_{\rho_{\theta(b) \theta(a)}\left(\xi_{b} \circ \xi_{\circ} \xi_{a}^{-1}\right)} \circ \phi_{\xi_{a}^{\prime}}\right)$ because of (3.2.5).

To see that each $f_{\alpha}^{\prime}$ is well-defined, suppose a point $x \in \widehat{\overline{U_{\alpha}}}$ lies in $\overline{\text { Range }\left(\phi_{\zeta_{a}}\right)} \cap \overline{\text { Range }\left(\phi_{\zeta_{b}}\right)}$ for some $\zeta_{a} \in T\left(U_{a}, U_{\alpha}\right), \zeta_{b} \in T\left(U_{b}, U_{\alpha}\right)$, and $\left(\phi_{\zeta_{a}}\right)^{-1}(x) \in K_{a},\left(\phi_{\zeta_{b}}\right)^{-1}(x) \in K_{b}$. We need to show that $\phi_{\zeta_{a}^{\prime}} \circ f_{a}^{\prime} \circ\left(\phi_{\zeta_{a}}\right)^{-1}(x)=\phi_{\zeta_{b}^{\prime}} \circ f_{b}^{\prime} \circ\left(\phi_{\zeta_{b}}\right)^{-1}(x)$, which can be derived as follows. For the convenience of expression, we set $f_{\alpha, a}^{\prime}=\phi_{\zeta_{a}^{\prime}} \circ f_{a}^{\prime} \circ\left(\phi_{\zeta_{a}}\right)^{-1}, f_{\alpha, b}^{\prime}=\phi_{\zeta_{b}^{\prime}} \circ f_{b}^{\prime} \circ\left(\phi_{\zeta_{b}}\right)^{-1}$, $f_{\theta(a)}^{\prime}=\phi_{\xi_{a}^{\prime}} \circ f_{a}^{\prime} \circ\left(\phi_{\xi_{a}}\right)^{-1}$ and $f_{\theta(b)}^{\prime}=\phi_{\xi_{b}^{\prime}} \circ f_{b}^{\prime} \circ\left(\phi_{\xi_{b}}\right)^{-1}$. Let $\mathbf{a}_{x} \in \Lambda\left(\zeta_{a}, \zeta_{b}^{-1}\right)$ be an element whose closure contains $\left(\phi_{\zeta_{a}}\right)^{-1}(x)$. Suppose $\mathbf{a}_{x} \subset L_{a,(\alpha, b)}^{i}$ for some $i \in \lambda(\alpha, b)$. Then

$$
\phi_{\rho_{\alpha \theta(a)}\left(\zeta_{a} \circ \xi_{a}^{-1}\right)}=\phi_{\rho_{\alpha \theta(b)}\left(\zeta_{b} \circ \xi_{b}^{-1}\right)} \circ \phi_{\rho_{\theta(b) \theta(a)}\left(\xi_{b} \circ\left(\zeta_{b}^{-1} \circ \zeta_{a}\left(\mathbf{a}_{x}\right)\right) \circ \xi_{a}^{-1}\right)} \text { on } W_{a,(\alpha, b)}^{i} \text {. }
$$


By the assumption that $\left\{f_{a}^{\prime}\right\} \in \mathcal{O}_{\left\{\rho_{b a}\right\}}\left(\left\{\underline{L_{a}^{0}}\right\},\left\{\underline{Q_{a}^{0}}\right\}\right)$, we have $f_{\theta(a)}^{\prime}\left(\phi_{\xi_{a} \circ \zeta_{a}^{-1}}(x)\right) \in W_{a,(\alpha, b)}^{i}$. Thus by (3.2.11), (3.2.13) and then (3.2.12),

$$
\begin{aligned}
& f_{\alpha, a}^{\prime}(x)=\phi_{\rho_{\alpha \theta(a)}\left(\zeta_{a} \circ \xi_{a}^{-1}\right)} \circ f_{\theta(a)}^{\prime} \circ \phi_{\xi_{a} \circ \zeta_{a}^{-1}}(x) \\
= & \phi_{\rho_{\alpha \theta(b)}\left(\zeta_{b} \circ \xi_{b}^{-1}\right)} \circ \phi_{\rho_{\theta(b) \theta(a)}\left(\xi_{b} \circ\left(\zeta_{b}^{-1} \circ \zeta_{a}\left(\mathbf{a}_{x}\right)\right) \circ \xi_{a}^{-1}\right)} \circ f_{\theta(a)}^{\prime} \circ \phi_{\xi_{a} \circ \zeta_{a}^{-1}}(x) \\
= & \phi_{\rho_{\alpha \theta(b)}\left(\zeta_{b} \circ \xi_{b}^{-1}\right)} \circ f_{\theta(b)}^{\prime} \circ \phi_{\xi_{b} \circ\left(\zeta_{b}^{-1} \circ \zeta_{a}\left(\mathbf{a}_{x}\right)\right) \circ \xi_{a}^{-1}} \circ \phi_{\xi_{a} \circ \zeta_{a}^{-1}}(x)=f_{\alpha, b}^{\prime}(x) .
\end{aligned}
$$

To verify $\phi_{\rho_{\beta \alpha}(\eta)} \circ f_{\alpha}^{\prime}=f_{\beta}^{\prime} \circ \phi_{\eta}$, let $x$ be any point in Domain $\left(\phi_{\eta}\right)$. We take a $\xi \in T\left(U_{a}, U_{\alpha}\right)$ for some index $a$ such that $x \in \operatorname{Range}\left(\phi_{\xi}\right)$ and $\left(\phi_{\xi}\right)^{-1}(x) \in K_{a}$. Set $\mathbf{a}_{x} \in \Lambda(\xi, \eta)$ to be the element containing $\left(\phi_{\xi}\right)^{-1}(x)$, and suppose $\mathbf{a}_{x} \subset L_{a,(\alpha, \beta)}^{i}$ for some $i \in \Lambda(\alpha, \beta)$. Then on $W_{a,(\alpha, \beta)}^{i}$ we have $\phi_{\rho_{\beta \theta(a)}\left(\eta \circ \xi\left(\mathbf{a}_{x}\right) \circ \xi_{a}^{-1}\right)}=\phi_{\rho_{\beta \alpha}(\eta)} \circ \phi_{\rho_{\alpha \theta(a)}\left(\xi \circ \xi_{a}^{-1}\right)}$. On the other hand, the assumption $\left\{f_{a}^{\prime}\right\} \in \mathcal{O}_{\left\{\rho_{b a}\right\}}\left(\left\{\underline{L_{a}^{0}}\right\},\left\{\underline{Q_{a}^{0}}\right\}\right)$ implies that $f_{\theta(a)}^{\prime} \circ \phi_{\xi_{a} \circ \xi^{-1}}(x) \in W_{a,(\alpha, \beta)}^{i}$. Hence by (3.2.11), we have

$$
\begin{aligned}
& \phi_{\rho_{\beta \alpha}(\eta)} \circ f_{\alpha}^{\prime}(x)=\phi_{\rho_{\beta \alpha}(\eta)} \circ \phi_{\rho_{\alpha \theta(a)}\left(\xi \circ \xi_{a}^{-1}\right)} \circ f_{\theta(a)}^{\prime} \circ \phi_{\xi_{a} \circ \xi^{-1}}(x) \\
= & \phi_{\rho_{\beta \theta(a)}\left(\eta \circ \xi\left(\mathbf{a}_{x}\right) \circ \xi_{a}^{-1}\right)} \circ f_{\theta(a)}^{\prime} \circ \phi_{\xi_{a} \circ \xi^{-1}}(x)=f_{\beta}^{\prime} \circ \phi_{\eta \circ \xi\left(\mathbf{a}_{x}\right) \circ \xi_{a}^{-1}} \circ \phi_{\xi_{a} \circ \xi^{-1}}(x) \\
= & f_{\beta}^{\prime} \circ \phi_{\eta}(x), \forall x \in \text { Domain }\left(\phi_{\eta}\right) .
\end{aligned}
$$

To verify $\rho_{\gamma \alpha}(\eta \circ \zeta(\mathbf{a}))=\rho_{\gamma \beta}(\eta) \circ \rho_{\beta \alpha}(\zeta)\left(\left\{f_{\alpha}^{\prime}\right\}(\mathbf{a})\right)$, it suffices to show that $f_{\alpha}^{\prime}(x) \in\left\{f_{\alpha}\right\}(\mathbf{a})$ if $x \in \mathbf{a}$. To this end, we pick a $U_{a}$ and a $\xi \in T\left(U_{a}, U_{\alpha}\right)$ such that $x \in \operatorname{Range}\left(\phi_{\xi}\right)$ and $\left(\phi_{\xi}\right)^{-1}(x) \in K_{a}$. Now observe that $\phi_{\xi^{\prime}} \circ f_{a} \circ\left(\phi_{\xi}\right)^{-1}(x)=f_{\alpha}(x) \in \underline{\left\{f_{\alpha}\right\}}(\mathbf{a})$, so that $f_{a} \circ\left(\phi_{\xi}\right)^{-1}(x) \in\left(\phi_{\xi^{\prime}}\right)^{-1}\left(\underline{\left\{f_{\alpha}\right\}}(\mathbf{a})\right)$. Let $\left(\phi_{\xi^{\prime}}\right)^{-1}\left(\underline{\left\{f_{\alpha}\right\}}(\mathbf{a})\right)=Q_{a,(\alpha, \beta, \gamma)}^{i}$ for some $i \in I(\alpha, \beta, \gamma)$. Then $\left(\phi_{\xi}\right)^{-1}(x)$ lies in $L_{a,(\alpha, \beta, \gamma)}^{i}$ by the virtue of definition. Now we appeal to the assumption that $\left\{f_{a}^{\prime}\right\} \in \mathcal{O}_{\left\{\rho_{b a}\right\}}\left(\left\{\underline{L_{a}^{0}}\right\},\left\{\underline{Q_{a}^{0}}\right\}\right)$ to conclude that $f_{a}^{\prime} \circ\left(\phi_{\xi}\right)^{-1}(x)$ lies in $Q_{a,(\alpha, \beta, \gamma)}^{i}$, which implies that $f_{\alpha}^{\prime}(x)=\phi_{\xi^{\prime}} \circ f_{a}^{\prime \circ}\left(\phi_{\xi}\right)^{-1}(x) \in \phi_{\xi^{\prime}}\left(Q_{a,(\alpha, \beta, \gamma)}^{i}\right) \subset\left\{f_{\alpha}\right\}(\mathbf{a})$.

To verify that $\left\{f_{a}^{\prime}\right\}$ is induced by $\left\{f_{\alpha}^{\prime}\right\}=\phi_{\bar{\gamma}}\left(\overline{\left\{f_{a}^{\prime}\right\}}\right)$ via the data $\bar{\gamma}=\left(\theta,\left\{\xi_{a}\right\},\left\{\xi_{a}^{\prime}\right\}\right)$, we observe that: (1) For $(3.2 .7 a), f_{a}^{\prime}(x)=\left(\phi_{\xi_{a}^{\prime}}\right)^{-1} \circ f_{\theta(a)}^{\prime} \circ \phi_{\xi_{a}}(x)$ holds for any $x \in K_{a}$ by the nature of construction; for the case when $x \in \widehat{U_{a}} \backslash K_{a}$, we pick a $U_{b}$ such that $x=\phi_{\xi}(y)$ for some $y \in K_{b}$ and $\xi \in T\left(U_{b}, U_{a}\right)$, and then apply (3.2.11) and (3.2.12). Hence $(3.2 .7 a)$ holds for $\left\{f_{a}^{\prime}\right\},\left\{f_{\alpha}^{\prime}\right\}$. (2) For $(3.2 .7 b)$, we need to verify that $f_{a}^{\prime}\left(\right.$ Domain $\left.\left(\phi_{\eta}\right)\right)$ is contained in $\mathbf{a}_{\eta}^{\bar{\gamma}}$, which is the unique element of $\Lambda\left(\xi_{a}^{\prime}, \rho_{\theta(b) \theta(a)}\left(\xi_{b} \circ \eta \circ \xi_{a}^{-1}\right),\left(\xi_{b}^{\prime}\right)^{-1}\right)$ that contains $f_{a}$ (Domain $\left.\left(\phi_{\eta}\right)\right)$. But this follows from the fact that Domain $\left(\phi_{\rho_{b a}(\eta)}\right)$ is contained in Domain $\left(\left(\phi_{\xi_{b}^{\prime}}\right)^{-1} \circ \phi_{\rho_{\theta(b) \theta(a)}\left(\xi_{b} \circ \eta \circ \xi_{a}^{-1}\right)} \circ \phi_{\xi_{a}^{\prime}}\right)$ because of $(3.2 .5)$, so that Domain $\left(\phi_{\rho_{b a}(\eta)}\right)=\mathbf{a}_{\eta}^{\bar{\gamma}}$, and the fact that $\phi_{\rho_{b a}(\eta)} \circ f_{a}^{\prime}=f_{b}^{\prime} \circ \phi_{\eta}$ so that $f_{a}^{\prime}\left(\right.$ Domain $\left.\left(\phi_{\eta}\right)\right) \subset$ Domain $\left(\phi_{\rho_{b a}(\eta)}\right)$. Hence $\left\{f_{a}^{\prime}\right\}$ is induced by $\left\{f_{\alpha}^{\prime}\right\}$ via the data $\bar{\gamma}=\left(\theta,\left\{\xi_{a}\right\},\left\{\xi_{a}^{\prime}\right\}\right)$. Note that as a corollary, $\phi_{\bar{\gamma}}$ is injective.

To verify $\phi_{\bar{\gamma}}\left(\left\{f_{a}\right\}\right)=\left\{f_{\alpha}\right\}$, we observe that if we let $\left\{\bar{f}_{\alpha}\right\}=\phi_{\bar{\gamma}}\left(\left\{f_{a}\right\}\right)$, then since both $\left\{f_{\alpha}\right\},\left\{\bar{f}_{\alpha}\right\}$ induce $\left\{f_{a}\right\}$ via the data $\bar{\gamma}=\left(\theta,\left\{\xi_{a}\right\},\left\{\xi_{a}^{\prime}\right\}\right)$, by Lemma 3.1.2, $\left\{f_{\alpha}\right\},\left\{\bar{f}_{\alpha}\right\}$ are conjugate to each other via some $\left\{g_{\alpha}\right\}$, where from the proof of Lemma 3.1.2, we notice that $g_{\alpha}=g_{\alpha}\left(\zeta_{a}\right)=\zeta_{a, 2}^{\prime} \circ\left(\zeta_{a, 1}^{\prime}\right)^{-1}(x), \forall x \in f_{a}\left(\widehat{U_{a}}\right)$. In the present case, one may take $\zeta_{a}=\xi_{a}$, and hence $\zeta_{a, 1}^{\prime}=\zeta_{a, 2}^{\prime}=\xi_{a}^{\prime}$. So $g_{\alpha}=1, \forall \alpha$. Thus $\left\{f_{\alpha}\right\}=\left\{\bar{f}_{\alpha}\right\}=\phi_{\bar{\gamma}}\left(\left\{f_{a}\right\}\right)$. (In this argument, we may accommodate the hypothesis in the proof of Lemma 3.1.2 that each $U_{\alpha}=\bigcup_{a \in I_{\alpha}} U_{a}$ for 
a subset $\left\{U_{a} \mid a \in I_{\alpha}\right\}$ of $\left\{U_{a}\right\}$ by passing to an induced homomorphism of $\left\{f_{a}\right\}$, which may not be admissible.)

It remains to show that $\phi_{\bar{\gamma}}$ is a continuous, open map.

Given any open subset $\mathcal{O}_{\left\{\rho_{\beta \alpha}\right\}}\left(\left\{\underline{K_{\alpha}}\right\},\left\{\underline{O_{\alpha}}\right\}\right)$ which contains $\left\{f_{\alpha, 0}^{\prime}\right\}=\phi_{\bar{\gamma}}\left(\left\{f_{a, 0}^{\prime}\right\}\right)$, we shall construct an open neighborhood $\mathcal{O}_{\left\{\rho_{b a}\right\}}\left(\left\{\underline{L_{a}}\right\},\left\{\underline{Q_{a}}\right\}\right)$ of $\left\{f_{a, 0}^{\prime}\right\}$, such that the open neigh-

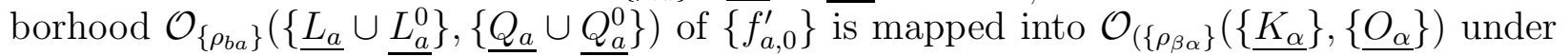
$\phi_{\bar{\gamma}}$. Write $\underline{K_{\alpha}}=\left\{K_{\alpha, i} \mid i \in I_{\alpha}\right\}, \underline{O_{\alpha}}=\left\{O_{\alpha, i} \mid i \in I_{\alpha}\right\}$. Let $a$ be any given index. Suppose $U_{a} \cap \overline{U_{\alpha}} \neq \emptyset$ for some index $\alpha$. Then for each $i \in I_{\alpha}, \overline{V_{a}} \cap \pi_{U_{\alpha}}\left(K_{\alpha, i}\right)$, where $\overline{V_{a}}$ is given in $K_{a}=\pi_{U_{a}}^{-1}\left(\overline{V_{a}}\right)$, is contained in finitely many components $\left\{U_{a,(\alpha, i)}^{j} \mid j \in \Lambda(\alpha, i)\right\}$ of $U_{a} \cap U_{\alpha}$. This is because $\overline{V_{a}} \cap \pi_{U_{\alpha}}\left(K_{\alpha, i}\right)$ is a compact subset of $U_{a} \cap U_{\alpha}$. Moreover, both $\cup_{j} \pi_{U_{a}}^{-1}\left(U_{a,(\alpha, i)}^{j}\right)$ and $\cup_{j} \pi_{U_{\alpha}}^{-1}\left(U_{a,(\alpha, i)}^{j}\right)$ have finitely many components because $U_{a}, U_{\alpha}$ are locally compact, Hausdorff, and the maps $\pi_{U_{a}}, \pi_{U_{\alpha}}$ are proper. Hence there exist finitely many $\xi_{k}, k \in I_{(\alpha, i)}$, such that if $x \in K_{\alpha, i}$ with $\pi_{U_{\alpha}}(x) \in \overline{V_{a}}=\pi_{U_{a}}\left(K_{a}\right)$, then there is a $\xi_{k}$ satisfying $x \in$ Range $\left(\phi_{\xi_{k}}\right)$ for some $k \in I_{(\alpha, i)}$. Set $L_{a,(\alpha, i)}^{k}=\left(\phi_{\xi_{k}}\right)^{-1}\left(K_{\alpha, i}\right) \cap K_{a}, Q_{a,(\alpha, i)}^{k}=\left(\phi_{\xi_{k}^{\prime}}\right)^{-1}\left(O_{\alpha, i}\right)$ for each $k \in I_{(\alpha, i)}$. We define $\underline{L_{a}}=\left\{L_{a,(\alpha, i)}^{k} \mid k \in I_{(\alpha, i)}, i \in I_{\alpha}, U_{a} \cap U_{\alpha} \neq \emptyset\right\}$ and $\underline{Q_{a}}=\left\{Q_{a,(\alpha, i)}^{k} \mid k \in I_{(\alpha, i)}, i \in I_{\alpha}, \bar{U}_{a} \cap U_{\alpha} \neq \emptyset\right\}$. Both $\underline{L_{a}}, \underline{Q_{a}}$ are finite sets because $\left\{U_{\alpha}\right\}$ is locally finite. Now the fact that $\left\{f_{\alpha, 0}^{\prime}\right\}=\phi_{\bar{\gamma}}\left(\left\{f_{a, 0}^{\prime}\right\}\right)$ is contained in $\mathcal{O}_{\left\{\rho_{\beta \alpha}\right\}}\left(\left\{\underline{K_{\alpha}}\right\},\left\{\underline{O_{\alpha}}\right\}\right)$ implies, by the nature of construction, that $\left\{f_{a, 0}^{\prime}\right\}$ is contained in $\mathcal{O}_{\left\{\rho_{b a}\right\}}\left(\left\{\underline{L_{a}}\right\},\left\{\underline{\underline{Q}_{a}}\right\}\right)$.

Now suppose $\left\{f_{a}^{\prime}\right\}$ is in $\mathcal{O}_{\left\{\rho_{b a}\right\}}\left(\left\{\underline{L_{a}} \cup \underline{L_{a}^{0}}\right\},\left\{\underline{Q_{a}} \cup \underline{Q_{a}^{0}}\right\}\right)$. Given any index $\alpha$, let $x \in K_{\alpha, i}$ be any point. Since $\left\{V_{a}\right\}$ is a cover of $\bar{X}$, there is a $U_{a}$ with a $\xi_{k} \in T\left(U_{a}, U_{\alpha}\right)$ for some $k \in I_{(\alpha, i)}$, such that $x \in$ Range $\left(\phi_{\xi_{k}}\right)$ and $\left(\phi_{\xi_{k}}\right)^{-1}(x) \in K_{a}$. By definition $\left(\phi_{\xi_{k}}\right)^{-1}(x) \in L_{a,(\alpha, i)}^{k}$, hence $f_{a}^{\prime} \circ\left(\phi_{\xi_{k}}\right)^{-1}(x) \in Q_{a,(\alpha, i)}^{k}=\left(\phi_{\xi_{k}^{\prime}}\right)^{-1}\left(O_{\alpha, i}\right)$, which implies that $f_{\alpha}^{\prime}(x)=\phi_{\xi_{k}^{\prime}} \circ f_{a}^{\prime} \circ$ $\left(\phi_{\xi_{k}}\right)^{-1}(x) \in O_{\alpha, i}$. Thus $\left\{f_{\alpha}^{\prime}\right\}=\phi_{\bar{\gamma}}\left(\left\{f_{a}^{\prime}\right\}\right)$ lies in $\mathcal{O}_{\left\{\rho_{\beta \alpha}\right\}}\left(\left\{\underline{K_{\alpha}}\right\},\left\{\underline{O_{\alpha}}\right\}\right)$. In other words, the

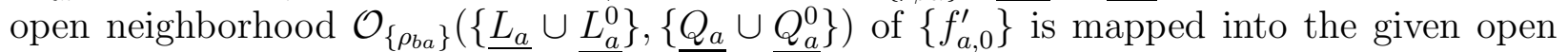
neighborhood $\mathcal{O}_{\left\{\rho_{\beta \alpha}\right\}}\left(\left\{\underline{K_{\alpha}}\right\},\left\{\underline{O_{\alpha}}\right\}\right)$ of $\left\{\overline{f_{\alpha, 0}^{\prime}}\right\}$ under the map $\phi_{\bar{\gamma}}$, hence $\phi_{\bar{\gamma}}$ is continuous.

To see that $\phi_{\bar{\gamma}}$ is an open map, we first show that the image of $\phi_{\bar{\gamma}}$ is an open subset of $\mathcal{O}_{\left\{\rho_{\beta \alpha}\right\}}$. To this end, suppose $\left\{f_{\alpha}^{\prime}\right\} \in \mathcal{O}_{\left\{\rho_{\beta \alpha}\right\}}$ satisfies $f_{\theta(a)}^{\prime}\left(\overline{\operatorname{Range}\left(\phi_{\xi_{a}}\right)}\right) \subset$ Range $\left(\phi_{\xi_{a}^{\prime}}\right)$ for each index $a$. Then an induced $\left\{\bar{f}_{a}\right\}$ of $\left\{f_{\alpha}^{\prime}\right\}$ is defined via $\bar{\gamma}=\left(\theta,\left\{\xi_{a}\right\},\left\{\xi_{a}^{\prime}\right\}\right)$. If furthermore, $\left\{\bar{f}_{a}\right\}$ lies in $\mathcal{O}_{\left\{\rho_{b a}\right\}}\left(\left\{\underline{L_{a}^{0}}\right\},\left\{\underline{Q_{a}^{0}}\right\}\right)$, then $\left\{\bar{f}_{\alpha}\right\}=\phi_{\bar{\gamma}}\left(\left\{\bar{f}_{a}\right\}\right)$ must equal $\left\{f_{\alpha}^{\prime}\right\}$, since both $\left\{\bar{f}_{\alpha}\right\},\left\{f_{\alpha}^{\prime}\right\}$ induce $\left\{\bar{f}_{a}\right\}$ via the same data $\bar{\gamma}=\left(\theta,\left\{\xi_{a}\right\},\left\{\xi_{a}^{\prime}\right\}\right)$, cf. Lemma 3.1.2.

Note that by (3.2.6), $\overline{\text { Range }\left(\phi_{\xi_{a}}\right)} \subset \widehat{U_{\theta(a)}}$ is compact. We set $K_{\alpha}^{0}=\left\{\overline{\operatorname{Range}\left(\phi_{\xi_{a}}\right)}, \phi_{\xi_{a}}(L) \mid\right.$ $\left.L \in \underline{L_{a}^{0}}\right\}, \underline{O_{\alpha}^{0}}=\left\{\right.$ Range $\left.\left(\phi_{\xi_{a}^{\prime}}\right), \phi_{\xi_{a}^{\prime}}(Q) \mid Q \in \underline{Q_{a}^{0}}\right\}$ if $\alpha=\theta(a)$, and define both to be empty sets otherwise. Then the argument in the preceding paragraph implies that $\mathcal{O}_{\left\{\rho_{\beta \alpha}\right\}}\left(\left\{K_{\alpha}^{0}\right\},\left\{\underline{O_{\alpha}^{0}}\right\}\right)$ is the image of the map $\phi_{\bar{\gamma}}$. Now for any $\mathcal{O}_{\left\{\rho_{b a}\right\}}\left(\left\{\underline{L_{a}}\right\},\left\{\underline{Q_{a}}\right\}\right)$, we define $\underline{K_{\alpha}}=\left\{\phi_{\xi_{a}} \overline{(L)} \mid L \bar{\epsilon} \underline{L_{a}}\right\}$, $O_{\alpha}=\left\{\phi_{\xi_{a}^{\prime}}(Q) \mid Q \in Q_{a}\right\}$ if $\alpha=\theta(a)$, and define both to be empty sets otherwise. Then $\overline{\mathcal{O}_{\left\{\rho_{b a}\right\}}}\left(\left\{\underline{L_{a}} \cup \underline{L_{a}^{0}}\right\},\left\{\underline{Q_{a}} \cup \underline{\cup Q_{a}^{0}}\right\}\right)$ is mapped onto $\mathcal{O}_{\left\{\rho_{\beta \alpha}\right\}}\left(\left\{\underline{K_{\alpha}} \cup \underline{K_{\alpha}^{0}}\right\},\left\{\underline{O_{\alpha}} \cup \underline{O_{\alpha}^{0}}\right\}\right)$ under $\phi_{\bar{\gamma}}$. Hence $\phi_{\bar{\gamma}}$ is an open map.

We summarize for the special case where $\tau$ is induced by $\sigma$ and (3.2.5) is satisfied, and $\bar{\Gamma}_{\sigma \tau}=\left\{\bar{\gamma}=\left(\theta,\left\{\xi_{a}\right\},\left\{\xi_{a}^{\prime}\right\}\right) \mid(3.2 .6),(3.2 .7 a),(3.2 .7 b)\right.$ are satisfied $\} \neq \emptyset$. Then each element $\bar{\gamma}$ of $\bar{\Gamma}_{\sigma \tau}$ is assigned with a local homeomorphism $\phi_{\bar{\gamma}}$, which clearly depends only on the equivalence class of $\bar{\gamma}$ defined by (3.1.4). According to Lemma 3.1.1, the set $\Gamma_{\sigma \tau}$ is defined to be the orbit space $\left(\Gamma_{\sigma \kappa} \times \Gamma_{\tau \kappa}\right) / G_{\kappa}$ where $\kappa$ is induced by $\tau$ with (3.1.2) satisfied. There is 
a natural surjective mapping $\bar{\Gamma}_{\sigma \tau} \rightarrow \Gamma_{\sigma \tau}$ given by $\bar{\gamma} \mapsto\left[\bar{\gamma} \circ \gamma_{0}, \gamma_{0}\right]$. The assignment $\bar{\gamma} \mapsto \phi_{\bar{\gamma}}$ factors through it, so that for any $\bar{\gamma} \in \bar{\Gamma}_{\sigma \tau}, \phi_{\bar{\gamma}}$ depends only on its image $\gamma \in \Gamma_{\sigma \tau}$.

More generally for any pair $(\sigma, \tau)$ of equivalent admissible homomorphisms, there are natural surjective mappings $\left(\bar{\Gamma}_{\sigma \hat{\kappa}} \times \bar{\Gamma}_{\tau \hat{\kappa}}\right) / G_{\hat{\kappa}} \rightarrow \Gamma_{\sigma \tau}$, where $\hat{\kappa}$ is any admissible homomorphism induced by $\sigma$, $\tau$ with $(3.2 .5),(3.2 .6)$ satisfied. These mappings are given by $\left[\bar{\gamma}_{1}, \bar{\gamma}_{2}\right] \mapsto$ $\left[\bar{\gamma}_{1} \circ \gamma_{0}, \bar{\gamma}_{2} \circ \gamma_{0}\right] \in\left(\Gamma_{\sigma \kappa} \times \Gamma_{\tau \kappa}\right) / G_{\kappa}=\Gamma_{\sigma \tau}$, where $\kappa$ is any homomorphism induced by $\hat{\kappa}$ with (3.1.2) satisfied with respect to $\sigma$ and $\tau$, and $\forall \gamma_{0} \in \Gamma_{\hat{\kappa} \kappa}$. Now given any $\gamma \in \Gamma_{\sigma \tau}$ which is the image of $\left[\bar{\gamma}_{1}, \bar{\gamma}_{2}\right] \in\left(\Gamma_{\sigma \hat{\kappa}} \times \Gamma_{\tau \hat{\kappa}}\right) / G_{\hat{\kappa}}$ under the above mappings, we define

$$
\phi_{\gamma}=\text { germ of } \phi_{\bar{\gamma}_{1}} \circ \phi_{\bar{\gamma}_{2}}^{-1} \text {. }
$$

In order to verify (3.2.4), we need one more piece of identities. Suppose $\bar{\gamma}_{1}, \bar{\gamma}_{2} \in \bar{\Gamma}_{\sigma \tau}$ where $\tau$ is induced by $\sigma$ and $(3.2 .5),(3.2 .6)$ is satisfied. Then $\phi_{\bar{\gamma}_{1}}^{-1} \circ \phi_{\bar{\gamma}_{2}}$ is a local homeomorphism from an open neighborhood of $\tau$ in $\mathcal{O}_{\left\{\rho_{b a}\right\}}$ to an open neighborhood of $\tau$ in $\mathcal{O}_{\left\{\rho_{b a}\right\}}$, leaving $\tau$ fixed. By Lemma 3.1.2, this must be induced by the action of an element in $G_{\tau}$. It can be computed as follows. Let $\bar{\gamma}_{1}=\bar{\gamma} \circ g_{1}, \bar{\gamma}_{2}=\bar{\gamma} \circ g_{2}$, where $g_{1}, g_{2} \in G_{\tau}$. Then $\phi_{\bar{\gamma}_{1}}^{-1} \circ \phi_{\bar{\gamma}_{2}}=\phi_{g_{1}^{-1} g_{2}}$, where $\phi_{g_{1}^{-1} g_{2}}$ is the action on $\mathcal{O}_{\left\{\rho_{b a}\right\}}$ induced by $g_{1}^{-1} g_{2} \in G_{\tau}$. On the other hand, for any homomorphism $\kappa$ induced by $\sigma, \tau$ with (3.1.2) satisfied, $\left(\bar{\gamma}_{1} \circ \gamma_{0}\right) \times\left(\bar{\gamma}_{2} \circ \gamma_{0}\right)=\left(\bar{\gamma} \circ g_{1} \circ \gamma_{0}\right) \times$ $\left(\bar{\gamma} \circ g_{2} \circ \gamma_{0}\right)=\left(\bar{\gamma} \circ \gamma_{0} \circ \epsilon\left(\gamma_{0}\right)^{-1}\left(g_{1}\right)\right) \times\left(\bar{\gamma} \circ \gamma_{0} \circ \epsilon\left(\gamma_{0}\right)^{-1}\left(g_{2}\right)\right)=\left(\epsilon\left(\gamma_{0}\right)^{-1}\left(g_{1}\right)\right)^{-1} \epsilon\left(\gamma_{0}\right)^{-1}\left(g_{2}\right)=$ $\epsilon\left(\gamma_{0}\right)^{-1}\left(g_{1}^{-1} g_{2}\right) \in G_{\kappa}$ for any $\gamma_{0} \in \Gamma_{\tau \kappa}$. Thus

$$
\phi_{\bar{\gamma}_{1}}^{-1} \circ \phi_{\bar{\gamma}_{2}}=\phi_{\epsilon\left(\gamma_{0}\right)\left(\left(\bar{\gamma}_{1} \circ \gamma_{0}\right) \times\left(\bar{\gamma}_{2} \circ \gamma_{0}\right)\right)}, \forall \bar{\gamma}_{1}, \bar{\gamma}_{2} \in \bar{\Gamma}_{\sigma \tau}, \gamma_{0} \in \Gamma_{\tau \kappa} .
$$

To verify (3.2.4), recall that if we write $\gamma=\left[\bar{\gamma}_{1} \circ \gamma_{0}, \bar{\gamma}_{2} \circ \gamma_{0}\right]$ and $\gamma^{\prime}=\left[\bar{\gamma}_{3} \circ \gamma_{0}, \bar{\gamma}_{4} \circ \gamma_{0}\right]$, we have $\gamma \circ \gamma^{\prime}=\left[\left(\bar{\gamma}_{1} \circ \gamma_{0}\right) \circ\left(\left(\bar{\gamma}_{2} \circ \gamma_{0}\right) \times\left(\bar{\gamma}_{3} \circ \gamma_{0}\right)\right),\left(\bar{\gamma}_{4} \circ \gamma_{0}\right)\right]=\left[\left(\bar{\gamma}_{1} \circ \epsilon\left(\gamma_{0}\right)\left(\left(\bar{\gamma}_{2} \circ \gamma_{0}\right) \times\left(\bar{\gamma}_{3} \circ \gamma_{0}\right)\right)\right) \circ \gamma_{0}, \bar{\gamma}_{4} \circ \gamma_{0}\right]$. Hence we deduce with the aid of (3.2.15) that

$$
\begin{aligned}
\phi_{\gamma \circ \gamma^{\prime}} & =\text { germ of } \phi_{\bar{\gamma}_{1} \circ \epsilon\left(\gamma_{0}\right)\left(\left(\bar{\gamma}_{2} \circ \gamma_{0}\right) \times\left(\bar{\gamma}_{3} \circ \gamma_{0}\right)\right)} \circ \phi_{\bar{\gamma}_{4}}^{-1} \\
& =\text { germ of } \phi_{\bar{\gamma}_{1}} \circ \phi_{\epsilon\left(\gamma_{0}\right)\left(\left(\bar{\gamma}_{2} \circ \gamma_{0}\right) \times\left(\bar{\gamma}_{3} \circ \gamma_{0}\right)\right)} \circ \phi_{\bar{\gamma}_{4}}^{-1} \\
& =\text { germ of } \phi_{\bar{\gamma}_{1}} \circ\left(\phi_{\bar{\gamma}_{2}}^{-1} \circ \phi_{\bar{\gamma}_{3}}\right) \circ \phi_{\bar{\gamma}_{4}}^{-1} \\
& =\text { germ of }\left(\phi_{\bar{\gamma}_{1}} \circ \phi_{\bar{\gamma}_{2}}^{-1}\right) \circ\left(\phi_{\bar{\gamma}_{3}} \circ \phi_{\bar{\gamma}_{4}}^{-1}\right)=\phi_{\gamma} \circ \phi_{\gamma^{\prime}}
\end{aligned}
$$

for any $\gamma \in \Gamma_{\sigma \tau}$ and $\gamma^{\prime} \in \Gamma_{\tau \kappa}$.

It is easy to check that if $\sigma=\phi_{\bar{\gamma}}(\tau)$ is also in $\mathcal{O}_{\left\{\rho_{b a}\right\}}$, then $\Gamma_{\sigma \tau} \subset G_{\left\{\rho_{b a}\right\}}$ and $\phi_{\gamma}$ is induced by the action of $G_{\left\{\rho_{b a}\right\}}$ on $\mathcal{O}_{\left\{\rho_{b a}\right\}}$.

Finally, for any $\tau^{\prime} \in$ Domain $\left(\phi_{\bar{\gamma}}\right), \sigma^{\prime}=\phi_{\bar{\gamma}}\left(\tau^{\prime}\right)$, the equivalence class $\gamma$ of $\bar{\gamma}$ is assigned with a $\gamma^{\prime} \in \Gamma_{\sigma^{\prime} \tau^{\prime}}$ such that the germ of $\phi_{\bar{\gamma}}$ at $\tau^{\prime}$ equals $\phi_{\gamma^{\prime}}$. Clearly, $\gamma^{\prime}$ is the equivalence class of $\bar{\gamma}$ in $\Gamma_{\sigma^{\prime} \tau^{\prime}}$.

\subsection{Proof of TheOrem 1.4}

Let $X, X^{\prime}$ be smooth orbifolds in the more general sense, ie., the group actions on a local uniformizing system need not to be effective. Assume without loss of generality that $X, X^{\prime}$ 
are connected. Note that $X, X^{\prime}$ are paracompact, locally compact and Hausdorff as defined in the beginning of $\S 3.2$. In particular, the space of maps $\left[X ; X^{\prime}\right]$ is canonically an orbispace.

We shall consider in this subsection, for any $r \geq 1$, the subset $\left[X ; X^{\prime}\right]^{r} \subset\left[X ; X^{\prime}\right]$ of maps of $C^{r}$ class, i.e., the set of equivalence classes of (admissible) homomorphisms $\left(\left\{f_{\alpha}\right\},\left\{\rho_{\beta \alpha}\right\}\right)$ where each $f_{\alpha}: \widehat{U_{\alpha}} \rightarrow \widehat{U_{\alpha}^{\prime}}$ is of $C^{r}$ class between domains in the Euclidean spaces.

First of all, we shall give a compatible topology to $\left[X ; X^{\prime}\right]^{r}$, stronger than the induced topology from $\left[X ; X^{\prime}\right]$. For this purpose, we introduce

$$
\mathcal{O}_{\left\{\rho_{\beta \alpha}\right\}}^{r}=\left\{\sigma \mid \sigma=\left(\left\{f_{\alpha}\right\},\left\{\rho_{\beta \alpha}\right\}\right) \text { is admissible and } f_{\alpha} \text { is } C^{r}, \forall \alpha\right\} .
$$

We give each $\mathcal{O}_{\left\{\rho_{\beta \alpha}\right\}}^{r}$ a topology that is generated by the subsets of the following type:

$$
\mathcal{O}_{\left\{\rho_{\beta \alpha}\right\}}^{r}\left(\left\{f_{\alpha}\right\},\left\{K_{\alpha}\right\},\left\{\epsilon_{\alpha}\right\}\right)=\left\{\left\{f_{\alpha}^{\prime}\right\} \mid\left\|f_{\alpha}^{\prime}-f_{\alpha}\right\|_{K_{\alpha}}^{C^{r}}<\epsilon_{\alpha}, \forall \alpha\right\},
$$

where $\left\{f_{\alpha}\right\} \in \mathcal{O}_{\left\{\rho_{\beta \alpha}\right\}}^{r}$, each $K_{\alpha}$ is a compact subset of $\widehat{U_{\alpha}}$, each $\epsilon_{\alpha}$ is a positive real number, and \|\|$_{K_{\alpha}}^{C^{r}}$ is the $C^{r}$-norm of a $C^{r}$-map between domains in the Euclidean spaces, defined over $K_{\alpha}$. Note that the set of subsets in (3.3.2) is actually a base of the topology on $\mathcal{O}_{\left\{\rho_{\beta \alpha}\right\}}^{r}$. The group $G_{\left\{\rho_{\beta \alpha}\right\}}$ in (3.2.2) acts continuously on $\mathcal{O}_{\left\{\rho_{\beta \alpha}\right\}}^{r}$ with the given topology, and the orbit space $\left[\mathcal{O}_{\left\{\rho_{\beta \alpha}\right\}}^{r}\right]$ is a subset of $\left[X ; X^{\prime}\right]^{r}$ by Lemma 3.1.2. We denote the set of orbits of the subsets in (3.3.2) by $\left[\mathcal{O}_{\left\{\rho_{\beta \alpha}\right\}}^{r}\left(\left\{f_{\alpha}\right\},\left\{K_{\alpha}\right\},\left\{\epsilon_{\alpha}\right\}\right)\right]$, which all together form a base of the quotient topology on $\left[\mathcal{O}_{\left\{\rho_{\beta \alpha}\right\}}^{r}\right]$. Finally, $\left[X ; X^{\prime}\right]^{r}$ is given a topology which is generated by the set of subsets

$$
\left[\mathcal{O}_{\left\{\rho_{\beta \alpha}\right\}}^{r}\left(\left\{f_{\alpha}\right\},\left\{K_{\alpha}\right\},\left\{\epsilon_{\alpha}\right\}\right)\right]
$$

for all possible data $\left\{\rho_{\beta \alpha}\right\},\left\{f_{\alpha}\right\},\left\{K_{\alpha}\right\},\left\{\epsilon_{\alpha}\right\}$. Call this topology the $C^{r}$-topology.

Proposition 3.3.1 The space of $C^{r}$-maps $\left[X ; X^{\prime}\right]^{r}$ with the $C^{r}$-topology is an orbispace under a canonical étale topological groupoid.

Proof The inclusions $\mathcal{O}_{\left\{\rho_{\beta \alpha}\right\}}^{r} \subset \mathcal{O}_{\left\{\rho_{\beta \alpha}\right\}}$ are continuous, and each $\phi_{\bar{\gamma}}$ in Lemma 3.2 .2 sends a $C^{r}$ admissible homomorphism to a $C^{r}$ admissible homomorphism. Hence each $\phi_{\bar{\gamma}}$ is a bijection from an open set in $\mathcal{O}_{\left\{\rho_{b a}\right\}}^{r}$ to an open set in $\mathcal{O}_{\left\{\rho_{\beta \alpha}\right\}}^{r}$. On the other hand, $\phi_{\bar{\gamma}}$ is clearly continuous with respect to the topology generated by the subsets in (3.3.2). Thus each $\phi_{\bar{\gamma}}$ is a local homeomorphism defined on the space $U^{r}=\sqcup \mathcal{O}_{\left\{\rho_{\beta \alpha}\right\}}^{r}$.

Consider the groupoid $\Gamma^{r}=\bigcup_{(\sigma, \tau)} \Gamma_{\sigma \tau}$ where $\sigma, \tau$ are running over the set of $C^{r}$ admissible homomorphisms. It is naturally identified with the set of germs of the elements in the pseudogroup generated by $\left\{\phi_{\bar{\gamma}}\right\}$, which acts on $U^{r}=\sqcup \mathcal{O}_{\left\{\rho_{\beta \alpha}\right\}}^{r}$ by local homeomorphisms. Given with the étale topology, $\Gamma^{r}$ becomes an étale topological groupoid, giving a canonical orbispace structure on $\left[X ; X^{\prime}\right]^{r}$, as one argues in the proof of Theorem 1.3.

We remark that when $X, X^{\prime}$ are smooth manifolds, $\left[X ; X^{\prime}\right]^{r}$ is simply the space of $C^{r}$-maps from $X$ to $X^{\prime}$ given with the Whitney topology, cf. [19].

Lemma 3.3.2 Let $E \rightarrow X^{\prime}$ be a smooth orbifold vector bundle over $X^{\prime}$.

- Given any $C^{r}$ homomorphism $\sigma$, there is a canonical pull-back $C^{r}$ orbifold vector bundle $E_{\sigma} \rightarrow X$, with a $C^{r}$ bundle morphism $\bar{\Phi}: E_{\sigma} \rightarrow E$ covering the equivalence class of $\sigma, \Phi: X \rightarrow X^{\prime}$. 
- For any $\gamma \in \Gamma_{\sigma \tau}$, there is a bundle isomorphism $\Xi(\gamma): E_{\tau} \rightarrow E_{\sigma}$, which is compatible with the bundle morphisms $\bar{\Phi}: E_{\sigma} \rightarrow E$ and satisfies $\Xi\left(\gamma_{2} \circ \gamma_{1}\right)=\Xi\left(\gamma_{2}\right) \circ \Xi\left(\gamma_{1}\right)$. In particular, $G_{\sigma}$ acts on $E_{\sigma}$, and $\Xi(\gamma)$ is $\epsilon(\gamma)$-equivariant.

Proof A smooth orbifold vector bundle of rank $n$ over $X^{\prime}$ may be given by a smooth homomorphism of topological groupoids $\mu: \Gamma\left\{U_{\alpha^{\prime}}^{\prime}\right\} \rightarrow G L_{n}(\mathbb{R})$. Suppose $\sigma=\left(\left\{f_{\alpha}\right\},\left\{\rho_{\beta \alpha}\right\}\right)$ : $\Gamma\left\{U_{\alpha}\right\} \rightarrow \Gamma\left\{U_{\alpha^{\prime}}^{\prime}\right\}$. We define the $C^{r}$ orbifold vector bundle $E_{\sigma} \rightarrow X$ to be the one given by the $C^{r}$ homomorphism $\mu \circ \sigma: \Gamma\left\{U_{\alpha}\right\} \rightarrow G L_{n}(\mathbb{R})$, where we represent $E \rightarrow X^{\prime}$ by $\mu: \Gamma\left\{U_{\alpha^{\prime}}^{\prime}\right\} \rightarrow G L_{n}(\mathbb{R})$.

More concretely, $\mu: \Gamma\left\{U_{\alpha^{\prime}}^{\prime}\right\} \rightarrow G L_{n}(\mathbb{R})$ is given by a collection of smooth maps $\left\{\mu_{\xi^{\prime}} \mid \xi^{\prime} \in\right.$ $\left.T\left(U_{\alpha^{\prime}}^{\prime}, U_{\beta^{\prime}}^{\prime}\right)\right\}$, where $\mu_{\xi^{\prime}}$ : Domain $\left(\phi_{\xi^{\prime}}\right) \rightarrow G L_{n}(\mathbb{R})$, such that

$$
\mu_{\eta^{\prime}}\left(\phi_{\xi^{\prime}}(x)\right) \mu_{\xi^{\prime}}(x)=\mu_{\eta^{\prime} \circ \xi^{\prime}(x)}(x), \forall x \in \phi_{\xi^{\prime}}^{-1}\left(\text { Domain }\left(\phi_{\eta^{\prime}}\right)\right) .
$$

The smooth orbifold vector bundle $E \rightarrow X^{\prime}$ is then given by a collection of local trivializations $\left\{\widehat{U_{\alpha^{\prime}}^{\prime}} \times \mathbb{R}^{n} \rightarrow \widehat{U_{\alpha^{\prime}}^{\prime}}\right\}$, with the action of $G_{U_{\alpha^{\prime}}^{\prime}}$ given by the formula

$$
g \cdot(x, v)=\left(g \cdot x, \mu_{g}(x)(v)\right), \forall g \in G_{U_{\alpha^{\prime}}^{\prime}},(x, v) \in \widehat{U_{\alpha^{\prime}}^{\prime}} \times \mathbb{R}^{n},
$$

and the set of transition functions $\left\{\varphi\left(\xi^{\prime}\right) \mid \xi^{\prime} \in T\left(U_{\alpha^{\prime}}^{\prime}, U_{\beta^{\prime}}^{\prime}\right)\right\}$ given by

$$
\varphi\left(\xi^{\prime}\right):(x, v) \mapsto\left(\phi_{\xi^{\prime}}(x), \mu_{\xi^{\prime}}(x)(v)\right), \forall x \in \text { Domain }\left(\phi_{\xi^{\prime}}\right), v \in \mathbb{R}^{n} .
$$

Likewise, $\mu^{\sigma}=\mu \circ \sigma$ is given by the collection of $C^{r}$ maps $\left\{\mu_{\xi}^{\sigma} \mid \xi \in T\left(U_{\alpha}, U_{\beta}\right)\right\}$, where $\mu_{\xi}^{\sigma}$ : Domain $\left(\phi_{\xi}\right) \rightarrow G L_{n}(\mathbb{R})$ is defined by $\mu_{\xi}^{\sigma}=\mu_{\rho_{\beta \alpha}(\xi)} \circ f_{\alpha}, \forall \xi \in T\left(U_{\alpha}, U_{\beta}\right)$. The $C^{r}$ orbifold vector bundle $E_{\sigma} \rightarrow X$ is defined by the collection of local trivializations $\left\{\widehat{U}_{\alpha} \times \mathbb{R}^{n} \rightarrow \widehat{U}_{\alpha}\right\}$, with the action of $G_{U_{\alpha}}$ given by the formula

$$
g \cdot(x, v)=\left(g \cdot x, \mu_{\rho_{\alpha}(g)}\left(f_{\alpha}(x)\right)(v)\right), \forall g \in G_{U_{\alpha}},(x, v) \in \widehat{U_{\alpha}} \times \mathbb{R}^{n},
$$

and the set of transition functions $\left\{\varphi(\xi) \mid \xi \in T\left(U_{\alpha}, U_{\beta}\right)\right\}$ given by

$$
\varphi(\xi):(x, v) \mapsto\left(\phi_{\xi}(x), \mu_{\rho_{\beta \alpha}(\xi)}\left(f_{\alpha}(x)\right)(v)\right), \forall x \in \text { Domain }\left(\phi_{\xi}\right), v \in \mathbb{R}^{n} .
$$

The $C^{r}$ bundle morphism $\bar{\Phi}: E_{\sigma} \rightarrow E$ is given by a collection of $C^{r}$ maps $\left\{\bar{f}_{\alpha}\right\}$, where

$$
\bar{f}_{\alpha}:(x, v) \mapsto\left(f_{\alpha}(x), v\right), \forall(x, v) \in \widehat{U_{\alpha}} \times \mathbb{R}^{n},
$$

such that $\left(\phi_{\rho_{\beta \alpha}(\xi)}, \mu_{\rho_{\beta \alpha}(\xi)}\right) \circ \bar{f}_{\alpha}=\bar{f}_{\beta} \circ\left(\phi_{\xi}, \mu_{\xi}^{\sigma}\right)$ for any $\alpha, \beta$ and $\xi \in T\left(U_{\alpha}, U_{\beta}\right)$.

As for the bundle isomorphisms $\Xi(\gamma): E_{\tau} \rightarrow E_{\sigma}, \gamma \in \Gamma_{\sigma \tau}$, we first consider the special case where $\tau$ is induced by $\sigma$ with (3.1.2) satisfied. Then given any $\gamma \in \Gamma_{\sigma \tau}$, we pick a representative $\bar{\gamma}=\left(\theta,\left\{\xi_{a}\right\},\left\{\xi_{a}^{\prime}\right\}\right)$ of $\gamma$, and define $\Xi(\bar{\gamma}): E_{\tau} \rightarrow E_{\sigma}$ by a collection of maps $\left\{\Xi(\bar{\gamma})_{a}\right\}$, where

$$
\Xi(\bar{\gamma})_{a}:(x, v) \mapsto\left(\phi_{\xi_{a}}(x), \mu_{\xi_{a}^{\prime}}\left(f_{a}(x)\right)(v)\right), \forall(x, v) \in \widehat{U_{a}} \times \mathbb{R}^{n} .
$$

The equivalence class of $\left\{\Xi(\bar{\gamma})_{a}\right\}$, which is the bundle isomorphism $\Xi(\bar{\gamma}): E_{\tau} \rightarrow E_{\sigma}$, depends only on the equivalence class of $\bar{\gamma}$ as defined by (3.1.4). We set $\Xi(\gamma)=\Xi(\bar{\gamma})$. More generally, for any pair $(\sigma, \tau)$ of equivalent homomorphisms, we define $\Xi(\gamma)=\Xi\left(\gamma_{1}\right) \circ \Xi\left(\gamma_{2}\right)^{-1}$ where $\gamma=\left[\gamma_{1}, \gamma_{2}\right]$. We leave the verification for the claimed properties of $\Xi(\gamma)$ in the lemma to the reader, which is straightforward. 
Now we come to the proof of Theorem 1.4.

Theorem 3.3.3 Suppose $X$ is compact.

(1) The space of $C^{r}$-maps $\left[X ; X^{\prime}\right]^{r}$ is naturally a smooth Banach orbifold. More concretely, $\left[X ; X^{\prime}\right]^{r}$ is Hausdorff and second countable, and there exists an open cover $\left\{\mathcal{O}_{i}\right\}$ of $\left[X ; X^{\prime}\right]^{r}$ with the following properties.

(i) Each $\mathcal{O}_{i}$ is associated with a triple $\left(\widehat{\mathcal{O}}_{i}, G_{i}, \pi_{i}\right)$, where $\widehat{\mathcal{O}}_{i}$ is an open ball in a Banach space, $G_{i}$ is a finite group acting linearly on $\widehat{\mathcal{O}}_{i}$, and $\pi_{i}: \widehat{\mathcal{O}}_{i} \rightarrow \mathcal{O}_{i}$ is a continuous map inducing a homeomorphism $\widehat{\mathcal{O}}_{i} / G_{i} \cong \mathcal{O}_{i}$.

(ii) There is an étale topological groupoid $\Gamma$ with the space of units $U=\bigsqcup_{i} \widehat{\mathcal{O}}_{i}$ such that (a) $\Gamma \backslash U=\left[X ; X^{\prime}\right]^{r}$, (b) the restriction of $\Gamma$ to each $\widehat{\mathcal{O}}_{i}$ is the product groupoid $G_{i} \times \widehat{\mathcal{O}}_{i}$, and (c) for each local section s of $\alpha: \Gamma \rightarrow U$, i.e., $\alpha \circ s=I d$, the map $\omega \circ s$ is a local diffeomorphism between Banach spaces.

(2) The set of $C^{l}$-maps is a dense subset of $\left[X ; X^{\prime}\right]^{r}$ for all $l \geq r$.

Proof (1) We fix an auxiliary Riemannian metric on $X^{\prime}$, whose existence is ensured by the paracompactness of $X^{\prime}$. This is to say that there is a family of Riemannian metrics $\left\{g_{i^{\prime}}\right\}$, where each $g_{i^{\prime}}$ is a $G_{U_{i^{\prime}}^{\prime}}$-equivariant Riemannian metric on the local chart $\widehat{U_{i^{\prime}}^{\prime}}$, with respect to which the local diffeomorphisms $\left\{\phi_{\xi^{\prime}} \mid \xi^{\prime} \in T\left(U_{i^{\prime}}^{\prime}, U_{j^{\prime}}^{\prime}\right)\right\}$ are isometric.

Let $\sigma=\left\{f_{\alpha}\right\} \in \mathcal{O}_{\left\{\rho_{\beta \alpha}\right\}}^{r}$ be any $C^{r}$ admissible homomorphism. By Lemma 3.3.2, there is a canonical pull-back $C^{r}$ orbifold vector bundle $E_{\sigma} \rightarrow X$, with a bundle morphism $\bar{\Phi}: E_{\sigma} \rightarrow$ $T X^{\prime}$ covering the equivalence class of $\sigma, \Phi: X \rightarrow X^{\prime}$. Note that $E_{\sigma} \rightarrow X$ inherits a natural metric from $T X^{\prime}$.

Denote by $C^{r}\left(E_{\sigma}\right)$ the space of $C^{r}$ sections of $E_{\sigma}$. We claim that $C^{r}\left(E_{\sigma}\right)$ is canonically identified with the set of admissible representatives of $C^{r}$ sections which are defined over an arbitrarily given locally finite, admissible cover $\left\{U_{\alpha}\right\}$, namely, the set of systems of local $C^{r}$ equivariant sections $\left\{s_{\alpha}: \widehat{U_{\alpha}} \rightarrow \widehat{U_{\alpha}} \times \mathbb{R}^{n}\right\}$, which can be extended over to the compact closure of $\widehat{U_{\alpha}}$ and satisfy $s_{\beta} \circ \phi_{\xi}(x)=\varphi(\xi) \circ s_{\alpha}(x), \forall x \in$ Domain $\left(\phi_{\xi}\right), \xi \in T\left(U_{\alpha}, U_{\beta}\right)$, cf. (3.3.7) for the definition of transition functions $\varphi(\xi)$. To see this, we first observe that the mapping $\left\{s_{\alpha}\right\} \mapsto\left[\left\{s_{\alpha}\right\}\right]$ is injective by Lemma 3.1.2. On the other hand, for any representative $\left\{s_{i}\right\}$ of a $C^{r}$ section which is defined over a refinement $\left\{U_{i}\right\}$ of $\left\{U_{\alpha}\right\}$, we are able to find a representative $\left\{s_{\alpha}\right\}$ of the $C^{r}$ section, which is defined over $\left\{U_{\alpha}\right\}$, as follows. Consider the corresponding representatives of the zero section, $\left\{0_{i}\right\}$ and $\left\{0_{\alpha}\right\}$. By Lemma 3.2.2, there is a local homeomorphism $\phi_{\bar{\gamma}}$ from an open neighborhood of $\left\{0_{i}\right\}$ to an open neighborhood of $\left\{0_{\alpha}\right\}$, which is clearly linear in this case. For any $\left\{s_{i}\right\}$, we pick a small $\epsilon>0$ such that $\left\{\epsilon s_{i}\right\}$ lies in the said open neighborhood of $\left\{0_{i}\right\}$ where $\phi_{\bar{\gamma}}$ is defined, and then we define $\left\{s_{\alpha}\right\}=\epsilon^{-1} \phi_{\bar{\gamma}}\left(\left\{\epsilon s_{i}\right\}\right)$. Here the existence of such an $\epsilon>0$ relies on the assumption that $X$ is compact. It is easy to see that $\left\{s_{i}\right\}$ is indeed equivalent to $\left\{s_{\alpha}\right\}$. Hence $C^{r}\left(E_{\sigma}\right)$ is canonically identified with the space of admissible compatible systems of local $C^{r}$ equivariant sections over an arbitrarily given locally finite, admissible cover.

We define a norm on $C^{r}\left(E_{\sigma}\right)$ as follows, which makes $C^{r}\left(E_{\sigma}\right)$ into a Banach space. For any $\left\{s_{\alpha}\right\} \in C^{r}\left(E_{\sigma}\right)$, we define its norm by $\left\|\left\{s_{\alpha}\right\}\right\|=\max _{\alpha}\left\|s_{\alpha}\right\|_{C^{r}}$, where $\left\|s_{\alpha}\right\|_{C^{r}}$ is the $C^{r}$-norm 
of the section $s_{\alpha}{ }^{2}$ defined using the metric on $E_{\sigma}$. Here the index set $\{\alpha\}$ is finite because $\left\{U_{\alpha}\right\}$ is locally finite and $X$ is compact.

Let $\tau$ be any admissible $C^{r}$ homomorphism which is equivalent to $\sigma$. According to Lemma 3.3.2, for any $\gamma \in \Gamma_{\sigma \tau}$, there is a canonical orbifold bundle isomorphism $\Xi(\gamma): E_{\tau} \rightarrow E_{\sigma}$. For simplicity, we still denote by $\Xi(\gamma)$ the induced isomorphism between the Banach spaces $C^{r}\left(E_{\tau}\right)$ and $C^{r}\left(E_{\sigma}\right)$, which is clearly norm-preserving. In particular, the isotropy group $G_{\sigma}$ defined in (3.1.1), which is a finite group in this case, acts linearly on $C^{r}\left(E_{\sigma}\right)$, preserving the norm of $C^{r}\left(E_{\sigma}\right)$. Moreover, the map $\Xi(\gamma): C^{r}\left(E_{\tau}\right) \rightarrow C^{r}\left(E_{\sigma}\right)$ is equivariant with respect to the isomorphism $\epsilon(\gamma): G_{\tau} \rightarrow G_{\sigma}$ constructed in Lemma 3.1.1.

Let $\widehat{\mathcal{O}_{\sigma}}(\epsilon)$ be the open ball of radius $\epsilon$ in $C^{r}\left(E_{\sigma}\right)$. Denote by Exp the exponential map on each $\widehat{U_{\alpha}^{\prime}}$. Then the map $\Theta_{\sigma}: \widehat{\mathcal{O}_{\sigma}}(\epsilon) \rightarrow \mathcal{O}_{\left\{\rho_{\beta \alpha}\right\}}^{r}$ defined by

$$
\Theta_{\sigma}(s)=\left\{\operatorname{Exp}_{f_{\alpha}(x)} s_{\alpha}\right\} \text { where } s=\left\{s_{\alpha}\right\}
$$

is an open embedding when $\epsilon>0$ is sufficiently small. Moreover, for any $\gamma \in \Gamma_{\sigma \tau}$ and any representative $\bar{\gamma}$ of $\gamma$, we have $\Theta_{\sigma} \circ \Xi(\gamma)=\phi_{\bar{\gamma}} \circ \Theta_{\tau}$ on the intersection of their domains, where $\phi_{\bar{\gamma}}$ is the local homeomorphism constructed in Lemma 3.2.2. In particular, the open embedding $\Theta_{\sigma}$ is equivariant with respect to the natural inclusion $G_{\sigma} \hookrightarrow G_{\left\{\rho_{\beta \alpha}\right\}}$.

Let $\mathcal{O}_{\sigma}(\epsilon)$ be the image of $\Theta_{\sigma}\left(\widehat{\mathcal{O}_{\sigma}}(\epsilon)\right)$ in $\left[\mathcal{O}_{\left\{\rho_{\beta \alpha}\right\}}^{r}\right]$, and let $\pi_{\sigma}: \widehat{\mathcal{O}_{\sigma}}(\epsilon) \rightarrow \mathcal{O}_{\sigma}(\epsilon)$ be the corresponding orbit map. We shall prove next that for sufficiently small $\epsilon>0$, the map $\pi_{\sigma}$ induces a homeomorphism between $\widehat{\mathcal{O}_{\sigma}}(\epsilon) / G_{\sigma}$ and $\mathcal{O}_{\sigma}(\epsilon)$.

Some digression first. (1) Observe that each $\widehat{U_{\alpha}^{\prime}}$ is partitioned into a finite disjoint union $\sqcup \widehat{U_{\alpha}^{\prime}}(H)$, where $\widehat{U_{\alpha}^{\prime}}(H)=\left\{x \in \widehat{U_{\alpha}^{\prime}} \mid G_{x}=H\right\}$. Here $H$ is a subgroup of $G_{U_{\alpha}^{\prime}}$, and $G_{x}$ is the stabilizer at $x$. Note that $\widehat{U_{\alpha}^{\prime}}(H)$ has the following property: if $g \in G_{U_{\alpha}^{\prime}}$ fixes a point in $\widehat{U_{\alpha}^{\prime}}(H)$, then $g$ must be an element of $H$, which implies that $g$ actually fixes the entire $\widehat{U_{\alpha}^{\prime}}(H)$. (2) For any $x \in \widehat{U_{\alpha}^{\prime}}$, there exists a $R_{x}>0$ with the following significance. For any $y, z \in \widehat{U_{\alpha}^{\prime}}$, if $y, z$ are joined to $x$ by a geodesic with length less than or equal to $R_{x}$, and $y=g \cdot z$ for some $g \in G_{U_{\alpha}^{\prime}}$, then $g$ lies in $G_{x}$. End of digression.

Now for any $(\alpha, H)$, if $\widehat{U_{\alpha}^{\prime}}(H)$ intersects with $f_{\alpha}\left(\widehat{U_{\alpha}}\right)$, we pick a point $x_{\alpha, H} \in \widehat{U_{\alpha}}$ such that $x_{\alpha, H}^{\prime}=f_{\alpha}\left(x_{\alpha, H}\right)$ lies in $\widehat{U_{\alpha}^{\prime}}(H)$. Since there are only finitely many $(\alpha, H)$, the set of positive numbers $R_{x_{\alpha, H}^{\prime}}$ has a minimum $R_{\sigma}>0$.

We require $\epsilon \leq R_{\sigma}$. Now suppose there are $s_{1}, s_{2} \in \widehat{\mathcal{O}_{\sigma}}(\epsilon)$ such that $\left[\Theta_{\sigma}\left(s_{1}\right)\right]=\left[\Theta_{\sigma}\left(s_{2}\right)\right]$ in $\left[\mathcal{O}_{\left\{\rho_{\beta \alpha}\right\}}^{r}\right]$. Then there is a $g=\left\{g_{\alpha}\right\} \in G_{\left\{\rho_{\beta \alpha}\right\}}$ such that $\Theta_{\sigma}\left(s_{2}\right)=g \cdot \Theta_{\sigma}\left(s_{1}\right)$. By the assumption that $\epsilon \leq R_{\sigma}$, we conclude that for any $\alpha, g_{\alpha}$ fixes each $x_{\alpha, H}^{\prime}$, hence fixes the entire $\widehat{U_{\alpha}^{\prime}}(H)$, where $H$ is a subgroup of $G_{U_{\alpha}^{\prime}}$ such that $\widehat{U_{\alpha}^{\prime}}(H)$ intersects with $f_{\alpha}\left(\widehat{U_{\alpha}}\right)$. But $f_{\alpha}\left(\widehat{U_{\alpha}}\right)$ is contained in the union of these $\widehat{U_{\alpha}^{\prime}}(H)$ 's, hence $f_{\alpha}\left(\widehat{U_{\alpha}}\right)$ is fixed by $g_{\alpha}$. This exactly means that $g=\left\{g_{\alpha}\right\}$ is an element of $G_{\sigma}$, which implies that $\pi_{\sigma}$ induces a homeomorphism between $\widehat{\mathcal{O}_{\sigma}}(\epsilon) / G_{\sigma}$ and $\mathcal{O}_{\sigma}(\epsilon)$.

We pick a collection of $\sigma_{i}$ and $\epsilon_{i} \in\left(0, R_{\sigma_{i}}\right]$ such that $\left\{\mathcal{O}_{\sigma_{i}}\left(\epsilon_{i}\right)\right\}$ is a cover of $\left[X ; X^{\prime}\right]^{r}$. We let $\mathcal{O}_{i}=\mathcal{O}_{\sigma_{i}}\left(\epsilon_{i}\right)$, and $\widehat{\mathcal{O}_{i}}=\widehat{\mathcal{O}_{\sigma_{i}}}\left(\epsilon_{i}\right), G_{i}=G_{\sigma_{i}}$ and $\pi_{i}=\pi_{\sigma_{i}}$. Then (1)-(i) of the theorem is proved.

\footnotetext{
${ }^{2}$ note that $s_{\alpha}$ can be extended over to the compact closure of $\widehat{U_{\alpha}}$ by assumption.
} 
We regard each $\widehat{\mathcal{O}}_{i}$ as an open subset of $\mathcal{O}_{\left\{\rho_{\beta \alpha}\right\}}^{r}$ for some data $\left\{\rho_{\beta \alpha}\right\}$ via the open embeddings defined in (3.3.10), and define $\Gamma$ to be the étale topological groupoid which is the restriction of the étale topological groupoid $\Gamma^{r}$ in Proposition 3.3.1 to $U=\sqcup \widehat{\mathcal{O}_{i}}$. Then $(a),(b)$ in (1)-(ii) of the theorem are immediate from the construction. As for $(c)$ of (1)-(ii), a little bit elementary Riemannian geometry shows that it boils down to the following local problem which can be easily checked: Let $\Omega$ be a compact domain in a Euclidean space. Any self-diffeomorphism of $\mathbb{R}^{N}$ induces a self-diffeomorphism of the Banach space $C^{r}\left(\Omega ; \mathbb{R}^{N}\right)$ of $C^{r}$-maps from $\Omega$ into $\mathbb{R}^{N}$.

It remains to check that $\left[X ; X^{\prime}\right]^{r}$ is Hausdorff and second countable.

To see that $\left[X ; X^{\prime}\right]^{r}$ is Hausdorff, suppose $\left\{\Phi_{n}\right\}$ is a sequence of $C^{r}$ maps such that $\lim _{n \rightarrow \infty} \Phi_{n}=\Phi$ and $\lim _{n \rightarrow \infty} \Phi_{n}=\Phi^{\prime}$. We need to show that $\Phi=\Phi^{\prime}$. First of all, at the level of induced maps between underlying spaces, we have $f=\lim _{n \rightarrow \infty} f_{n}, f^{\prime}=\lim _{n \rightarrow \infty} f_{n}$, where $f_{n}, f$ and $f^{\prime}$ are the induced maps of $\Phi_{n}, \Phi$, and $\Phi^{\prime}$ respectively. Since $X^{\prime}$ is Hausdorff, we have $f=f^{\prime}$. This allows us to find a cover of local charts $\left\{U_{\alpha}\right\}$ on $X$, locally finite and admissible, and a subset of local charts $\left\{U_{\alpha^{\prime}}^{\prime}\right\}$ on $X^{\prime}$, with a correspondence $U_{\alpha} \mapsto U_{\alpha}^{\prime} \in$ $\left\{U_{\alpha^{\prime}}^{\prime}\right\}$, such that there is an admissible $C^{r}$ homomorphism $\sigma=\left(\left\{f_{\alpha}^{(1)}\right\},\left\{\rho_{\beta \alpha}^{(1)}\right\}\right): \Gamma\left\{U_{\alpha}\right\} \rightarrow$ $\Gamma\left\{U_{\alpha^{\prime}}^{\prime}\right\}$ representing $\Phi$, and there is an admissible $C^{r}$ homomorphism $\tau=\left(\left\{f_{\alpha}^{(2)}\right\},\left\{\rho_{\beta \alpha}^{(2)}\right\}\right)$ : $\Gamma\left\{U_{\alpha}\right\} \rightarrow \Gamma\left\{U_{\alpha^{\prime}}^{\prime}\right\}$ representing $\Phi^{\prime}$. On the other hand, since both $G_{\left\{\rho_{\beta \alpha}^{(1)}\right\}}$ and $G_{\left\{\rho_{\beta \alpha}^{(2)}\right\}}$ are finite groups, there are sequences of representatives $\left\{\sigma_{n}\right\},\left\{\tau_{n}\right\}$ of $\Phi_{n}$, such that (1) for any $n, \sigma_{n} \in \mathcal{O}_{\left\{\rho_{\beta \alpha}^{(1)}\right\}}^{r}, \tau_{n} \in \mathcal{O}_{\left\{\rho_{\beta \alpha}^{(2)}\right\}}^{r}$, and (2) $\lim _{n \rightarrow \infty} \sigma_{n}=\sigma, \lim _{n \rightarrow \infty} \tau_{n}=\tau$. By Lemma 3.1.2, the fact that both $\sigma_{n}, \tau_{n}$ represent the map $\Phi_{n}$ implies that there are $g_{\alpha, n} \in G_{U_{\alpha}^{\prime}}$ such that $\sigma_{n}$ is conjugate to $\tau_{n}$ by $\left\{g_{\alpha, n}\right\}$. But each $G_{U_{\alpha}^{\prime}}$ is finite, and $\max _{\alpha}\left|G_{U_{\alpha}^{\prime}}\right|<\infty$. Hence there is a subsequence $n_{i} \rightarrow \infty$, with $g_{\alpha, n_{i}}=g_{\alpha}$ independent of $n_{i}$. Now it is clear that $\sigma$ is conjugate to $\tau$ by $\left\{g_{\alpha}\right\}$, and hence $\Phi=\Phi^{\prime}$ as desired.

As for the second countability of $\left[X ; X^{\prime}\right]^{r}$, we fix a countable cover of local charts $\mathcal{U}_{0}^{\prime} \subset \mathcal{U}^{\prime}$ of $X^{\prime}$, and a countable base of local charts $\mathcal{U}_{0} \subset \mathcal{U}$ of $X$. The existence of $\mathcal{U}_{0}^{\prime}$ and $\mathcal{U}_{0}$ follows from the second countability of $X^{\prime}$ and $X$. Now observe that: (a) because $\mathcal{U}_{0}, \mathcal{U}_{0}^{\prime}$ are countable, so is the set $\Lambda$ of data $\left(\left\{U_{\alpha}\right\},\left\{U_{\alpha}^{\prime}\right\},\left\{\rho_{\beta \alpha}\right\}\right)$, where $\left\{U_{\alpha}\right\}$ is a finite subset of $\mathcal{U}_{0}$, with an assignment $U_{\alpha} \mapsto U_{\alpha}^{\prime} \in \mathcal{U}_{0}^{\prime}$, and $\rho_{\beta \alpha}: T\left(U_{\alpha}, U_{\beta}\right) \rightarrow T\left(U_{\alpha}^{\prime}, U_{\beta}^{\prime}\right)$ is a mapping between finite sets, (b) the set $\left\{\left[\mathcal{O}_{\left\{\rho_{\beta \alpha}\right\}}^{r}\right] \mid\left(\left\{U_{\alpha}\right\},\left\{U_{\alpha}^{\prime}\right\},\left\{\rho_{\beta \alpha}\right\}\right) \in \Lambda\right\}$ is a cover of $\left[X ; X^{\prime}\right]^{r}$, and (c) each space $\mathcal{O}_{\left\{\rho_{\beta \alpha}\right\}}^{r}$ is second countable - the validity of this last assertion boils down to the second countability of the Banach space $C^{r}\left(\Omega ; \mathbb{R}^{N}\right)$ of $C^{r}$ maps from a compact domain $\Omega$ in a Euclidean space into $\mathbb{R}^{N}$. The second countability of $\left[X ; X^{\prime}\right]^{r}$ follows easily from the observations (a), (b) and (c) above.

(2) In order to prove that the set of $C^{l}$-maps is dense in $\left[X ; X^{\prime}\right]^{r}$ for any $l \geq r$, it suffices to show that for any $\mathcal{O}_{\left\{\rho_{j i}\right\}}^{r}$, where we may assume $i, j \in\{1,2, \cdots, N\}$ for some $N>0$ since $X$ is compact, and for any open neighborhood $\mathcal{O}_{\left\{\rho_{j i}\right\}}^{r}\left\{\left\{f_{i}\right\},\left\{K_{i}\right\},\left\{\epsilon_{i}\right\}\right)$ of $\sigma=\left\{f_{i}\right\}$, there is a $\tau=\left\{f_{i}^{\prime}\right\} \in \mathcal{O}_{\left\{\rho_{j i}\right\}}^{r}\left(\left\{f_{i}\right\},\left\{K_{i}\right\},\left\{\epsilon_{i}\right\}\right)$ which is of $C^{l}$ class. Since the set of indexes $i$ is finite, we may assume $\epsilon_{i}=\epsilon$, which is independent of $i$, by taking a smaller neighborhood. $\tau=\left\{f_{i}^{\prime}\right\}$ will be defined by induction on $i$. First, we pick any $C^{l}$-map $h_{1}$ satisfying $\left\|h_{1}-f_{1}\right\|_{C^{r}}<\epsilon$, where the $C^{r}$-norm is taken over the closure of $\widehat{U}_{1}$. Then we make the $C^{l}$ map $\rho_{1}$-equivariant 
by setting $f_{1}^{\prime}(x)=\frac{1}{\left|G_{U_{1}}\right|} \sum_{g \in G_{U_{1}}} \rho_{1}\left(g^{-1}\right) h_{1}(g \cdot x)$. Clearly $f_{1}^{\prime}$ is $\rho_{1}$-equivariant and satisfies $\left\|f_{1}^{\prime}-f_{1}\right\|_{C^{r}}<\epsilon$. Now assume $f_{i}^{\prime}$ is defined and satisfies $\left\|f_{i}^{\prime}-f_{i}\right\|_{C^{r}}<\epsilon$ for $1 \leq i \leq n-1$, and $\left(\left\{f_{i}^{\prime}\right\},\left\{\rho_{j i}\right\}\right)$, where $1 \leq i \leq n-1$, is a homomorphism. We shall construct $f_{n}^{\prime}$ as follows. Let $A \subset$ closure of $\widehat{U_{n}}$ be the set of $x$ where there exists a $\xi \in T\left(U_{n}, U_{i}\right)$ for some $i<n$, such that $x \in \overline{\text { Domain }\left(\phi_{\xi}\right)}$. For any $x \in A$, we define $f_{n}^{\prime}(x)=\phi_{\rho_{\text {in }}(\xi)}^{-1} \circ f_{i}^{\prime} \circ \phi_{\xi}(x)$, which is independent of the choice of $\xi$ because $\left(\left\{f_{i}^{\prime}\right\},\left\{\rho_{j i}\right\}\right)$, where $1 \leq i \leq n-1$, is a homomorphism. Moreover, $A$ is invariant under the action of $G_{U_{n}}$, and $f_{n}^{\prime}$ over $A$ is $\rho_{n}$-equivariant, of $C^{l}$ class, and satisfies $\left\|f_{n}^{\prime}-f_{n}\right\|_{A}^{C^{r}}<\epsilon$. Now we simply extend $f_{n}^{\prime}$ to a $C^{l}$-map over the closure of $\widehat{U_{n}}$, making it $\rho_{n}$-equivariant and satisfy $\left\|f_{n}^{\prime}-f_{n}\right\|_{C^{r}}<\epsilon$. To see that $\left(\left\{f_{i}^{\prime}\right\},\left\{\rho_{j i}\right\}\right)$, where $1 \leq i \leq n$, is a homomorphism, it suffices to check that $\rho_{k i}(\eta \circ \xi(\mathbf{a}))=\rho_{k j}(\eta) \circ \rho_{j i}(\xi)\left(\left\{f_{i}^{\prime}\right\}(\mathbf{a})\right)$ for any $1 \leq i, j, k \leq n$ and any $\mathbf{a} \in \Lambda(\xi, \eta)$. When $\epsilon>0$ is sufficiently small, $\left\{f_{i}^{\prime}\right\} \overline{(\mathbf{a})=\left\{f_{i}\right\}}(\mathbf{a})$, from which the above equation follows. Now by induction, $\tau=\left\{f_{i}^{\prime}\right\} \in \mathcal{O}_{\left\{\rho_{j i}\right\}}$, where $\overline{1 \leq i} \leq N$, is defined, which is of $C^{l}$ class and lies in the given neighborhood $\mathcal{O}_{\left\{\rho_{j i}\right\}}^{r}\left(\left\{f_{i}\right\},\left\{K_{i}\right\},\left\{\epsilon_{i}\right\}\right)$. Hence the set of $C^{l}$-maps is dense in $\left[X ; X^{\prime}\right]^{r}$.

The proof of Theorem 3.3.3 is completed.

The rest of this subsection is concerned with two results which are useful in the theory of pseudoholomorphic curves in symplectic orbifolds, cf. eg. [7, 8].

For the first one, let $X, Y, Z$ be compact smooth orbifolds. Consider the mapping of composition $(\Phi, \Psi) \mapsto \Psi \circ \Phi$, where $\Phi \in[X ; Y]^{r}, \Psi \in[Y ; Z]^{r}$. Similar argument as in the proof of Proposition 3.2.3 shows that it defines a smooth 'map' from $[X ; Y]^{r} \times[Y ; Z]^{r}$ to $[X ; Z]^{r}$. But note that the term 'map' is not quite clearly defined here because it is not known whether $[X ; Y]^{r} \times[Y ; Z]^{r},[X ; Z]^{r}$ as smooth Banach orbifolds satisfy $(\mathrm{C} 2)$ or not, except for the case where they are actually smooth Banach manifolds. However, as far as applications are concerned, it suffices to only consider finite dimensional suborbifolds of $[X ; Y]^{r} \times[Y ; Z]^{r}$ and $[X ; Z]^{r}$.

Proposition 3.3.4 Let $M, N$ be finite dimensional suborbifolds of $[X ; Y]^{r} \times[Y ; Z]^{r}$ and $[X ; Z]^{r}$ such that $\Psi \circ \Phi \in N$ for any $(\Phi, \Psi) \in M$. Then there is a canonically defined smooth map of orbifolds from $M$ to $N$, which induces $(\Phi, \Psi) \mapsto \Psi \circ \Phi$ between the underlying spaces.

Proof For each $(\Phi, \Psi) \in M$, pick admissible homomorphisms $\sigma, \tau$ representing $\Phi, \Psi$ respectively, such that $\kappa \equiv \tau \circ \sigma$ is defined. Then as in the proof of Proposition 3.2.3, there are positive real numbers $\epsilon_{\sigma}, \epsilon_{\tau}$ and $\epsilon_{\kappa}$, and open balls of Banach spaces $\widehat{\mathcal{O}_{\sigma}}\left(\epsilon_{\sigma}\right), \widehat{\mathcal{O}_{\tau}}\left(\epsilon_{\tau}\right)$ and $\widehat{\mathcal{O}_{\kappa}}\left(\epsilon_{\kappa}\right)$ as defined in the proof of Theorem 3.3.3, such that for any $\sigma^{\prime} \in \widehat{\mathcal{O}_{\sigma}}\left(\epsilon_{\sigma}\right), \tau^{\prime} \in \widehat{\mathcal{O}_{\tau}}\left(\epsilon_{\tau}\right)$, $\kappa^{\prime} \equiv \tau^{\prime} \circ \sigma^{\prime}$ is defined and lies in $\widehat{\mathcal{O}_{\kappa}}\left(\epsilon_{\kappa}\right)$. Let $\left(\widehat{U_{(\sigma, \tau)}}, G_{(\sigma, \tau)}\right),\left(\widehat{U_{\kappa}}, G_{\kappa}\right)$ be local uniformizing systems of $M, N$ at $(\Phi, \Psi), \Psi \circ \Phi$, which are induced from $\left(\widehat{\mathcal{O}_{\sigma}}\left(\epsilon_{\sigma}\right) \times \widehat{\mathcal{O}_{\tau}}\left(\epsilon_{\tau}\right), G_{\sigma} \times G_{\tau}\right)$, $\left.\widehat{\left(\mathcal{O}_{\kappa}\right.}\left(\epsilon_{\kappa}\right), G_{\kappa}\right)$ respectively. By Proposition 2.1.3, we may choose $\epsilon_{\sigma}, \epsilon_{\tau}$ and $\epsilon_{\kappa}$ sufficiently small so that $(\mathrm{C} 1),(\mathrm{C} 2)$ are satisfied for these local uniformizing systems of $M, N$.

Next we define a homomorphism of topological groupoids from $\Gamma\left\{U_{(\sigma, \tau)}\right\}$ to $\Gamma\left\{U_{\kappa}\right\}$. More concretely, let $\left(\sigma_{1}^{\prime}, \tau_{1}^{\prime}\right) \in \widehat{U_{\left(\sigma_{1}, \tau_{1}\right)}},\left(\sigma_{2}^{\prime}, \tau_{2}^{\prime}\right) \in \widehat{U_{\left(\sigma_{2}, \tau_{2}\right)}}$ such that $\sigma_{1}^{\prime}, \sigma_{2}^{\prime}$ and $\tau_{1}^{\prime}, \tau_{2}^{\prime}$ are equivalent, and let $\gamma_{1} \in \Gamma_{\sigma_{2}^{\prime} \sigma_{1}^{\prime}}, \gamma_{2} \in \Gamma_{\tau_{2}^{\prime} \tau_{1}^{\prime}}$. Set $\kappa_{1}^{\prime} \equiv \tau_{1}^{\prime} \circ \sigma_{1}^{\prime} \in \widehat{U_{\kappa_{1}}}, \kappa_{2}^{\prime} \equiv \tau_{2}^{\prime} \circ \sigma_{2}^{\prime} \in \widehat{U_{\kappa_{2}}}$. We shall define a 
$\gamma \in \Gamma_{\kappa_{2}^{\prime} \kappa_{1}^{\prime}}$ such that $\left(\left(\gamma_{1}, \gamma_{2}\right),\left(\sigma_{1}^{\prime}, \tau_{1}^{\prime}\right)\right) \mapsto\left(\gamma, \kappa_{1}^{\prime}\right)$ is a groupoid homomorphism from $\Gamma\left\{U_{(\sigma, \tau)}\right\}$ to $\Gamma\left\{U_{\kappa}\right\}$. Note that it suffices to only consider the case where $\sigma_{1}^{\prime}, \tau_{1}^{\prime}$ are induced by $\sigma_{2}^{\prime}, \tau_{2}^{\prime}$. To fix the notation, let $\sigma_{1}^{\prime}=\left(\left\{f_{a}\right\},\left\{\rho_{b a}\right\}\right): \Gamma\left\{U_{a}\right\} \rightarrow \Gamma\left\{V_{a}\right\}, \sigma_{2}^{\prime}=\left(\left\{f_{\alpha}\right\},\left\{\rho_{\beta \alpha}\right\}\right): \Gamma\left\{U_{\alpha}\right\} \rightarrow$ $\Gamma\left\{V_{\alpha}\right\}, \tau_{1}^{\prime}=\left(\left\{h_{s}\right\},\left\{\zeta_{t s}\right\}\right): \Gamma\left\{V_{s}\right\} \rightarrow \Gamma\left\{W_{s}\right\}$, and $\tau_{2}^{\prime}=\left(\left\{h_{\mu}\right\},\left\{\zeta_{\nu \mu}\right\}\right): \Gamma\left\{V_{\mu}\right\} \rightarrow \Gamma\left\{W_{\mu}\right\}$. Let $\imath: a \mapsto s, \jmath: \alpha \mapsto \mu$ be the mappings of indexes such that $\kappa_{1}^{\prime}=\left(\left\{h_{\imath(a)} \circ f_{a}\right\},\left\{\zeta_{\imath(b) \imath(a)} \circ \rho_{b a}\right\}\right)$ and $\kappa_{2}^{\prime}=\left(\left\{h_{\jmath(\alpha)} \circ f_{\alpha}\right\},\left\{\zeta_{\jmath(\beta) \jmath(\alpha)} \circ \rho_{\beta \alpha}\right\}\right)$. Finally, let $\gamma_{1}, \gamma_{2}$ be represented by $\left(\theta_{1},\left\{\xi_{a}\right\},\left\{\xi_{a}^{\prime}\right\}\right)$, $\left(\theta_{2},\left\{\eta_{s}\right\},\left\{\eta_{s}^{\prime}\right\}\right)$, where $\theta_{1}: a \mapsto \alpha, \theta_{2}: s \mapsto \mu, \xi_{a} \in T\left(U_{a}, U_{\theta_{1}(a)}\right), \xi_{a}^{\prime} \in T\left(V_{a}, V_{\theta_{1}(a)}\right)$, $\eta_{s} \in T\left(V_{s}, V_{\theta_{2}(s)}\right)$ and $\eta_{s}^{\prime} \in T\left(W_{s}, W_{\theta_{2}(s)}\right)$. Note that $V_{a}=V_{\imath(a)}, V_{\theta_{1}(a)}=V_{\jmath\left(\theta_{1}(a)\right)}$, thus for each index $a$, set $\hat{\xi}_{a} \equiv \eta_{\imath(a)} \circ\left(\xi_{a}^{\prime}\right)^{-1} \in T\left(V_{\jmath\left(\theta_{1}(a)\right)}, V_{\theta_{2}(\imath(a))}\right)$. Then it is easy to check that $\kappa_{1}^{\prime}$ is induced by $\kappa_{2}^{\prime}$ via $\left(\theta_{1},\left\{\xi_{a}\right\},\left\{\left(\zeta_{\theta_{2}(\imath(a))_{\jmath}\left(\theta_{1}(a)\right)}\left(\hat{\xi}_{a}\right)\right)^{-1} \circ \eta_{\imath(a)}^{\prime}\right\}\right)$. We define $\gamma$ to be the corresponding equivalence class in $\Gamma_{\kappa_{2}^{\prime} \kappa_{1}^{\prime}}$. It is a routine exercise to check that $\left(\left(\gamma_{1}, \gamma_{2}\right),\left(\sigma_{1}^{\prime}, \tau_{1}^{\prime}\right)\right) \mapsto\left(\gamma, \kappa_{1}^{\prime}\right)$ is well-defined, and that it is a groupoid homomorphism from $\Gamma\left\{U_{(\sigma, \tau)}\right\}$ to $\Gamma\left\{U_{\kappa}\right\}$. We leave the details to the reader.

The equivalence class of the above homomorphism from $\Gamma\left\{U_{(\sigma, \tau)}\right\}$ to $\Gamma\left\{U_{\kappa}\right\}$ defines a map from $M$ to $N$ (cf. Lemma 2.2.1), which is smooth because $\left(\sigma_{1}^{\prime}, \tau_{1}^{\prime}\right) \mapsto \kappa_{1}^{\prime}$ is smooth, and it induces $(\Phi, \Psi) \mapsto \Psi \circ \Phi$ between the underlying spaces of $M, N$. Finally, observe that the map is canonically defined such that it is actually independent of $\left\{U_{(\sigma, \tau)}\right\}$ and $\left\{U_{\kappa}\right\}$.

For the second result, let $X, X^{\prime}$ be compact smooth orbifolds. Suppose $M$ is a finite dimensional suborbifold of $\left[X ; X^{\prime}\right]^{r}$, which consists of only smooth maps from $X$ to $X^{\prime}$.

Proposition 3.3.5 There is a canonically defined smooth map from $M \times X$ to $X^{\prime}$ which induces the evaluation map $(\Phi, p) \mapsto|\Phi|(p)$ between the underlying spaces, where $|\Phi|$ is the map between the underlying spaces of $X, X^{\prime}$ induced by $\Phi$.

Proof For each $\Phi \in M$, pick an admissible homomorphism $\sigma=\left(\left\{f_{\alpha}\right\},\left\{\rho_{\beta \alpha}\right\}\right): \Gamma\left\{U_{\alpha}(\sigma)\right\} \rightarrow$ $\Gamma\left\{U_{\alpha^{\prime}}^{\prime}(\sigma)\right\}$. Let $\left(\widehat{U_{\sigma}}, G_{\sigma}\right)$ be a local uniformizing system of $M$ at $\Phi$, which is induced from $\left(\widehat{\mathcal{O}_{\sigma}}\left(\epsilon_{\sigma}\right), G_{\sigma}\right)$ for some sufficiently small $\epsilon_{\sigma}>0$. Furthermore, we may assume that $(\mathrm{C} 1)$, (C2) are satisfied for the local uniformizing systems $\left\{\left(\widehat{U_{\sigma}}, G_{\sigma}\right)\right\}$, cf. Proposition 2.1.3.

Next we define a groupoid homomorphism from $\Gamma\left\{U_{\sigma} \times U_{\alpha}(\sigma)\right\}$ to $\Gamma\left\{U_{\alpha^{\prime}}^{\prime}(\sigma)\right\}$. To this end, for any $\left(\tau^{\prime}, x\right) \in \widehat{U_{\tau}} \times \widehat{U_{a}(\tau)}$, where $\tau^{\prime}=\left(\left\{f_{a}^{\prime}\right\},\left\{\rho_{b a}\right\}\right)$, and for any $\gamma \in \Gamma_{\sigma^{\prime} \tau^{\prime}}$ and $\xi \in T\left(U_{a}(\tau), U_{\alpha}(\sigma)\right)$ such that $x \in$ Domain $\left(\phi_{\xi}\right)$, where $\sigma^{\prime}=\left(\left\{f_{\alpha}^{\prime}\right\},\left\{\rho_{\beta \alpha}\right\}\right) \in \widehat{U_{\sigma}}$ and $\gamma$ is represented by $\left(\theta,\left\{\xi_{a}\right\},\left\{\xi_{a}^{\prime}\right\}\right)$, we let $\xi^{\prime}=\rho_{\alpha \theta(a)}\left(\xi \circ \xi_{a}^{-1}\right) \circ \xi_{a}^{\prime}\left(f_{a}^{\prime}(x)\right) \in T\left(U_{a}^{\prime}(\tau), U_{\alpha}^{\prime}(\sigma)\right)$. Then it is a routine exercise to check that $\left((\gamma, \xi),\left(\tau^{\prime}, x\right)\right) \mapsto\left(\xi^{\prime}, f_{a}^{\prime}(x)\right)$ defines a groupoid homomorphism from $\Gamma\left\{U_{\sigma} \times U_{\alpha}(\sigma)\right\}$ to $\Gamma\left\{U_{\alpha^{\prime}}^{\prime}(\sigma)\right\}$.

The corresponding map from $M \times X$ to $X^{\prime}$ is smooth because $\left(\tau^{\prime}, x\right) \mapsto f_{a}^{\prime}(x)$ is smooth. It is clear that it induces the evaluation map $(\Phi, p) \mapsto|\Phi|(p)$ between the underlying spaces, and that it is canonically defined such that it is independent of the choices made on $\left\{\widehat{U_{\sigma}}\right\}$. Details are left to the reader.

We end here with a discussion concerning group actions on orbifolds. Let $\mathcal{G}$ be a Lie group and $X$ be a compact smooth orbifold in the classical sense (ie. all local group actions on the uniformizing systems are effective). By a smooth, effective left action of $\mathcal{G}$ on $X$, we mean a smooth injective map $\iota: \mathcal{G} \rightarrow[X ; X]^{r}$ for some $r$ such that the image of $\iota$ consists of only 
smooth maps, and $\iota(1)=I d, \iota\left(g_{1} g_{2}\right)=\iota\left(g_{1}\right) \circ \iota\left(g_{2}\right)$ for any $g_{1}, g_{2} \in \mathcal{G}$. Note that the image of $\iota$ is contained in an open Banach submanifold of $[X ; X]^{r}$, so that $\iota$ being a smooth map is simply in the usual sense. Then as in Proposition 3.3.5, there is a canonical smooth map of orbifolds from $\mathcal{G} \times X$ to $X$, which induces the map $(g, p) \mapsto|\iota(g)|(p)$ between the underlying spaces. (Note that the induced map defines a continuous left action of $\mathcal{G}$ on the underlying space of $X$.) On the other hand, given any smooth map of orbifolds $\psi: \mathcal{G} \times X \rightarrow X$, there is an associated map $\iota: \mathcal{G} \rightarrow[X ; X]^{r}$, with $\iota(g): X \rightarrow X$ being defined by the restriction of $\psi$ on the suborbifold $\{g\} \times X$. Clearly $\iota(g) \in[X ; X]^{r}$ is smooth. Suppose furthermore, $\iota(1)=I d$ and $\iota\left(g_{1} g_{2}\right)=\iota\left(g_{1}\right) \circ \iota\left(g_{2}\right)$ for any $g_{1}, g_{2} \in \mathcal{G}$, and $\iota$ is injective. Then $\iota: \mathcal{G} \rightarrow[X ; X]^{r}$ defines a smooth, effective left action of $\mathcal{G}$ on $X$. The only thing remains to be verified is that $\iota$ is a smooth map. To see this, recall that $X$ is compact, so that for any $g \in \mathcal{G}$, there is an open neighborhood $U$ of $g$ in $\mathcal{G}$, such that the map $\psi: \mathcal{G} \times X \rightarrow X$ is represented by a homomorphism $\sigma=\left(\left\{f_{a}\right\},\left\{\rho_{b a}\right\}\right): \Gamma\left\{U_{a}\right\} \rightarrow \Gamma\left\{U_{a^{\prime}}^{\prime}\right\}$, where a neighborhood of $\{g\} \times X$ in $\mathcal{G} \times X$ is covered by a subset $\left\{U \times V_{i}\right\}$ of $\left\{U_{a}\right\}$. Let $\theta: i \mapsto a$ be the mapping of indexes such that $U \times V_{i}=U_{\theta(i)}$. Then for any $h \in U, \iota(h) \in[X ; X]^{r}$ is represented by the homomorphism $\tau_{h}=\left(\left\{f_{\theta(i)}(h, \cdot)\right\},\left\{\rho_{\theta(j) \theta(i)}\right\}\right)$. Hence $\iota$ is a smooth map.

Finally, suppose $\iota: \mathcal{G} \rightarrow[X ; X]^{r}$ is a smooth, effective left action of $\mathcal{G}$ on $X$, and $M \subset$ $\left[X ; X^{\prime}\right]^{r}$ is a finite dimensional suborbifold such that for any $\Phi \in M, g \in \mathcal{G}, \Phi \circ \iota(g) \in M$. Then as in Proposition 3.3.4, there is a canonically defined smooth map of orbifolds from $\mathcal{G} \times M$ to $M$, which induces $(g, \Phi) \mapsto \Phi \circ \iota\left(g^{-1}\right)$ between the underlying spaces. Clearly, the corresponding map $\jmath: \mathcal{G} \rightarrow[M ; M]^{r}$ satisfies $\jmath(1)=I d, \jmath\left(g_{1} g_{2}\right)=\jmath\left(g_{1}\right) \circ \jmath\left(g_{2}\right)$ for any $g_{1}, g_{2} \in \mathcal{G}$. If furthermore, when $M$ is a genuine orbifold, $M$ is compact and is an orbifold in the classical sense, and $\jmath$ is injective, then $\jmath$ defines a smooth, effective left action of $\mathcal{G}$ on $M$ of reparametrization. (Note that when $M$ is a manifold, the canonical map $\mathcal{G} \times M \rightarrow M$ defines a smooth action of $\mathcal{G}$ on $M$ in the usual sense.)

\subsection{Proof of Theorem 1.5}

We begin with some basic facts about complete Riemannian orbifolds, which are straightforward generalizations of the corresponding results for smooth manifolds.

For any connected Riemannian orbifold $X$, one can define a distance function $d$ on it as follows. For any $p, q \in X$, the distance $d(p, q)$ between $p$ and $q$ is the infinimum of the lengths of all piecewise $C^{1}$ paths in $X$ joining $p$ and $q$. By definition a piecewise $C^{1}$ path in $X$ joining $p$ and $q$ is a map $[a, b] \rightarrow X$, represented by a homomorphism $\sigma=\left(\left\{f_{\alpha}\right\},\left\{\rho_{\beta \alpha}\right\}\right)$ where each $f_{\alpha}:\left(a_{\alpha}, b_{\alpha}\right) \rightarrow \widehat{U_{\alpha}}$ is piecewise $C^{1}$, such that for the induced map $f:[a, b] \rightarrow X$ between the underlying spaces, $f(a)=p, f(b)=q$. Its length is defined to be the integral $\int_{a}^{b}\left|\frac{d f_{\alpha}}{d t}\right| d t$, where the norm $\left|\frac{d f_{\alpha}}{d t}\right|$ is defined with respect to the given Riemannian metric on $X$. A $C^{1}$ path in $X$ is a parametric geodesic if it is represented by a homomorphism $\sigma=\left(\left\{f_{\alpha}\right\},\left\{\rho_{\beta \alpha}\right\}\right)$

where each $f_{\alpha}:\left(a_{\alpha}, b_{\alpha}\right) \rightarrow \widehat{U_{\alpha}}$ is a parametric geodesic. A Riemannian metric on an orbifold is called 'complete' if every parametric geodesic in $X$ has $(-\infty, \infty)$ as its maximal domain.

The following basic results concerning complete Riemannian metrics on smooth manifolds, cf. e.g. pp. 172 in [23], are readily extended to the orbifold case. 
(1) For any two points in a connected complete Riemannian manifold, there is a minimizing geodesic connecting them.

(2) The following are equivalent:

- The Riemannian metric is complete.

- The metric space defined by the distance function $d$ is complete.

- Every bounded subset is precompact.

Now let $X, X^{\prime}$ be any complete Riemannian orbifolds where $X$ is also compact. We fix some distance functions $d, d^{\prime}$ on $X$ and $X^{\prime}$ respectively. Given any $\Phi \in\left[X ; X^{\prime}\right]^{r}$, which is represented by a $C^{r}$ admissible homomorphism $\sigma=\left(\left\{f_{\alpha}\right\},\left\{\rho_{\beta \alpha}\right\}\right)$, the differential $d \Phi$ is a $C^{r-1}$ section of the orbifold vector bundle $\operatorname{End}\left(T X, E_{\sigma}\right) \rightarrow X$, where $E_{\sigma} \rightarrow X$ is the canonical pull-back $C^{r}$ orbifold vector bundle constructed in Lemma 3.3.2 with $E=T X^{\prime}$. Note that $\operatorname{End}\left(T X, E_{\sigma}\right) \rightarrow X$ inherits a natural metric from both $X$ and $X^{\prime}$, hence $d \Phi$ as a $C^{r-1}$ section has a natural $C^{r-1}$-norm, which is denoted by $\|d \Phi\|_{C^{r-1}}$, and is simply $\max _{\alpha}\left\|d f_{\alpha}\right\|_{C^{r-1}}$ in terms of $\sigma=\left(\left\{f_{\alpha}\right\},\left\{\rho_{\beta \alpha}\right\}\right)$. Now pick an arbitrary point $p_{0}^{\prime} \in X^{\prime}$, and denote by $f: X \rightarrow X^{\prime}$ the induced map of $\Phi$ between the underlying spaces. We define the $C^{r}$-norm of $\Phi$ by

$$
\|\Phi\|_{C^{r}}=\max _{p \in X} d^{\prime}\left(p_{0}^{\prime}, f(p)\right)+\|d \Phi\|_{C^{r-1}}
$$

With the proceeding understood, we have

Theorem 3.4.1 Let $\left\{\Phi_{n} \mid n \geq 1\right\}$ be any sequence of $C^{r}$-maps such that $\left\|\Phi_{n}\right\|_{C^{r}} \leq C$ for some constant $C>0$ independent of $n$. Then there exists a $C^{r-1}-$ map $\Phi_{0}$, and a subsequence $\left\{\Phi_{n_{i}}\right\}$ of $\left\{\Phi_{n}\right\}$ such that $\Phi_{n_{i}}$ converges to $\Phi_{0}$ in the $C^{r-1}$-topology.

Proof Let $f_{n}: X \rightarrow X^{\prime}$ be the induced map of $\Phi_{n}$ between the underlying spaces. The assumption that $\left\|\Phi_{n}\right\|_{C^{r}} \leq C$ (with $r \geq 1$ ) for some constant $C>0$ independent of $n$ implies that all $f_{n}(X)$ are contained in a fixed bounded subset of $X^{\prime}$, which is precompact by the completeness of the Riemannian metric on $X^{\prime}$, and moreover, there exists a constant $C^{\prime}>0$ independent of $n$ such that $d^{\prime}\left(f_{n}(p), f_{n}(q)\right) \leq C^{\prime} d(p, q), \forall p, q \in X, \forall n$. By the classical Arzela-Ascoli theorem, there is a subsequence of $\left\{f_{n}\right\}$, still denoted by $\left\{f_{n}\right\}$ for simplicity, and there is a continuous map $f_{0}: X \rightarrow X^{\prime}$, such that $f_{n}$ converges to $f_{0}$ in the $C^{0}$-topology.

The image $f_{0}(X)$ is a compact subset of $X^{\prime}$. Thus we can cover $f_{0}(X)$ by a finite set $\left\{U_{\alpha^{\prime}}^{\prime}\right\}$ of local charts on $X^{\prime}$. Consider the cover of open subsets $\left\{f_{0}^{-1}\left(U_{\alpha^{\prime}}^{\prime}\right)\right\}$ of $X$. There exists a finite cover $\left\{U_{\alpha}\right\}$ of local charts on $X$, such that each $U_{\alpha}$ is admissible and the closure of $\widehat{U_{\alpha}}, \widehat{\overline{U_{\alpha}}}$, is a closed ball in the Euclidean space. Moreover, $\left\{\overline{U_{\alpha}}\right\}$ is a refinement of $\left\{f_{0}^{-1}\left(U_{\alpha^{\prime}}^{\prime}\right)\right\}$, which in particular means that there is a correspondence $U_{\alpha} \mapsto U_{\alpha}^{\prime} \in\left\{U_{\alpha^{\prime}}^{\prime}\right\}$ such that $f_{0}\left(\overline{U_{\alpha}}\right) \subset U_{\alpha}^{\prime}$. For sufficiently large $n$, we have $f_{n}\left(\overline{U_{\alpha}}\right) \subset U_{\alpha}^{\prime}$ since $f_{n}$ converges to $f_{0}$ in $C^{0}$-topology.

A digression is in order. Suppose $\Phi: X \rightarrow X^{\prime}$ is any map of orbispaces where $X=Y / G$, $X^{\prime}=Y^{\prime} / G^{\prime}$ are global quotients. Then by the covering space theory developed in the sequel [6], $\Phi$ is represented by a pair $(f, \rho):(Y, G) \rightarrow\left(Y^{\prime}, G^{\prime}\right)$, where $f$ is $\rho$-equivariant, if and only if under the induced homomorphism $\Phi_{*}: \pi_{1}(X) \rightarrow \pi_{1}\left(X^{\prime}\right)$, the subgroup $\pi_{1}(Y) \subset \pi_{1}(X)$ is sent into the subgroup $\pi_{1}\left(Y^{\prime}\right) \subset \pi_{1}\left(X^{\prime}\right)$, cf. $\S 2.4$ of [6]. End of digression.

Observe that because of $f_{n}\left(\overline{U_{\alpha}}\right) \subset U_{\alpha}^{\prime}$ for sufficiently large $n$, the restriction of $\Phi_{n}$ to the subspace $\overline{U_{\alpha}}$ is a map into the subspace $U_{\alpha}^{\prime}$ of $X^{\prime}$. Since each $\widehat{U_{\alpha}}$ is a closed ball, we have 
$\left(\Phi_{n}\right)_{*}\left(\pi_{1}\left(\widehat{U_{\alpha}}\right)\right)=\{1\} \subset \pi_{1}\left(\widehat{U_{\alpha}^{\prime}}\right)$. Hence the restriction of each $\Phi_{n}$ to $\overline{U_{\alpha}}$ for $n$ sufficiently large is represented by a pair $\left(f_{\alpha}^{(n)}, \rho_{\alpha}^{(n)}\right):\left(\widehat{\overline{U_{\alpha}}}, G_{U_{\alpha}}\right) \rightarrow\left(\widehat{U_{\alpha}^{\prime}}, G_{U_{\alpha}^{\prime}}\right)$, where $f_{\alpha}^{(n)}$ is $\rho_{\alpha}^{(n)}$-equivariant.

By Lemma 3.1.3, there exist mappings $\rho_{\beta \alpha}^{(n)}: T\left(U_{\alpha}, U_{\beta}\right) \rightarrow T\left(U_{\alpha}^{\prime}, U_{\beta}^{\prime}\right)$ with $\rho_{\alpha \alpha}^{(n)}=\rho_{\alpha}^{(n)}$, such that $\sigma_{n}=\left(\left\{f_{\alpha}^{(n)}\right\},\left\{\rho_{\beta \alpha}^{(n)}\right\}\right)$ is a $C^{r}$ admissible homomorphism representing the map $\Phi_{n}$. On the other hand, there is an infinite sequence $n_{i} \rightarrow \infty$ such that $\rho_{\beta \alpha}^{\left(n_{i}\right)}=\rho_{\beta \alpha}$ for some $\rho_{\beta \alpha}: T\left(U_{\alpha}, U_{\beta}\right) \rightarrow T\left(U_{\alpha}^{\prime}, U_{\beta}^{\prime}\right)$ independent of $n_{i}$ for all indexes $\alpha, \beta$, because there are only finitely many indexes and each $\rho_{\beta \alpha}^{(n)}$ is a mapping between two finite sets independent of $n$. Finally, since for each $\alpha,\left\{f_{\alpha}^{\left(n_{i}\right)}\right\}$ has bounded $C^{r}$-norms, we apply the classical Arzela-Ascoli theorem to conclude that there is a $C^{r-1}$-map $f_{\alpha}^{(0)}: \widehat{U_{\alpha}} \rightarrow \widehat{U_{\alpha}^{\prime}}$ such that a subsequence of $f_{\alpha}^{\left(n_{i}\right)}$, still denoted by $f_{\alpha}^{\left(n_{i}\right)}$, converges to $f_{\alpha}^{(0)}$ in $C^{r-1}$-topology.

To see that $\left(\left\{f_{\alpha}^{(0)}\right\},\left\{\rho_{\beta \alpha}\right\}\right)$ is a homomorphism, it suffices to check that $\rho_{\gamma \alpha}(\eta \circ \xi(\mathbf{a}))=$ $\rho_{\gamma \beta}(\eta) \circ \rho_{\beta \alpha}(\xi)\left(\underline{\left\{f_{\alpha}^{(0)}\right\}}(\mathbf{a})\right)$ for any $\mathbf{a} \in \Lambda(\xi, \eta)$. When $n_{i}$ is sufficiently large, $\underline{\left\{f_{\alpha}^{(0)}\right\}}(\mathbf{a})=$ $\left\{f_{\alpha}^{\left(n_{i}\right)}\right\}(\mathbf{a})$, from which the above equation follows.

Putting everything together, we may conclude that the sequence $\sigma_{n_{i}}=\left(\left\{f_{\alpha}^{\left(n_{i}\right)}\right\},\left\{\rho_{\beta \alpha}\right\}\right)$ of $C^{r}$ admissible homomorphisms converges to the $C^{r-1}$ admissible homomorphism $\sigma_{0}=$ $\left(\left\{f_{\alpha}^{(0)}\right\},\left\{\rho_{\beta \alpha}\right\}\right)$ in $\mathcal{O}_{\left\{\rho_{\beta \alpha}\right\}}^{r-1}$. This means that the corresponding maps $\Phi_{n_{i}}=\left[\sigma_{n_{i}}\right]$ converges to a $C^{r-1}$-map $\Phi_{0}=\left[\sigma_{0}\right]$ in the $C^{r-1}$-topology.

\section{REFERENCES}

[1] M.R. Bridson and A. Haefliger, Metric spaces of non-positive curvature, Grundlehren der mathematischen Wissenschaften 319, Springer-Verlag, 1999.

[2] K.S. Brown, Cohomology of groups, Graduate Texts in Mathematics 87, Springer-Verlag, 1982.

[3] M. Chas and D. Sullivan, String topology, arXiv: math. GT/9911159.

[4] W. Chen, A homotopy theory of orbispaces, math. AT/0102020.

[5] W. Chen, The loop space of an orbifold, talk at Workshop on Mathematical Aspects of Orbifold String Theory, Univ. of Wisconsin-Madison, May 4-8, 2001.

[6] W. Chen, On a notion of maps between orbifolds, II. homotopy and CW-complex, preprint.

[7] W. Chen, Orbifold adjunction formula and symplectic cobordisms between lens spaces, Geometry and Topology 8, 701-734 (2004).

[8] W. Chen, Smooth s-cobordisms of elliptic 3-manifolds, J. Diff. Geom. (2006), to appear.

[9] W. Chen, Pseudoholomorphic curves in four-orbifolds and some applications, in Geometry and Topology of Manifolds, Boden, H.U. et al ed., Fields Institute Communications 47, pp. 11-37. Amer. Math. Soc., Providence, RI, 2005

[10] W. Chen and Y. Ruan, Orbifold Gromov-Witten theory, in "Orbifolds in Mathematics and Physics", 25-85, Edited by Adem et al., Contemporary Mathematics 310, Amer. Math. Soc., Providence, RI, 2002.

[11] W. Chen and Y. Ruan, A new cohomology theory of orbifolds, Communications in Mathematical Physics 248 no.1 (2004), 1-31.

[12] L. Dixon, J. Harvey, C. Vafa and E. Witten, Strings on orbifolds, I,II, Nucl. Phys. B261 (1985) 678-, B274 (1986) 285-.

[13] K. Guruprasad and A. Haefliger, Closed geodesics on orbifolds, arXiv:math.DG/0306238 v1.

[14] A. Haefliger, Homotopy and integrability, in "Manifolds - Amsterdam 1970", 133-163, Lecture Notes in Math. 197, Springer, Berlin.

[15] A. Haefliger, Groupoides d'holonomie et classifiants, Astérisque 116 (1984), 70-97. 
[16] A. Haefliger, Complexes of groups and orbihedra, in "Group theory from a geometrical viewpoint, 26 March - 6 April 1990, ICTP, Trieste", World Scientific (1991), 504-540.

[17] A. Haefliger, Extension of complexes of groups, Ann. Inst. Fourier, Grenoble 42, 1-2 (1992), 275-311.

[18] M. Hilsum and G. Skandalis, Morphismes K-orientés d'espaces de feuilles et fonctorialité en théorie de

Kasparov (d'après une conjecture d'A. Connes), Ann. Sci. École Norm. Sup. (4) 20 (1987), no.3, 325-390

[19] M.W. Hirsch, Differential topology, Grad. Text in Math. 33 (1976), Springer-Verlag.

[20] F. Hirzebruch and T. Höfer, On the Euler number of an orbifold, Math. Ann. 286 (1990), 255-260.

[21] T. Kawasaki, The signature theorem for V-manifolds, Topology, 17 (1978), 75-83.

[22] T. Kawasaki, The index of elliptic operators over V-manifolds, Nagoya Math. J. 84, 135-157 (1981)

[23] S. Kobayashi and K. Nomizu, Foundations of differential geometry, I, by John Wiley \& Sons, Inc., 1963.

[24] K. Liu, talk at Workshop on Mathematical Aspects of Orbifold String Theory, Univ. of WisconsinMadison, May 4-8, 2001.

[25] E. Lupercio and B. Uribe, Loop groupoids, gerbes, and twisted sectors on orbifolds, in "Orbifolds in Mathematics and Physics", 163-184, Edited by Adem et al., Contemporary Mathematics 310, Amer. Math. Soc., Providence, RI, 2002.

[26] D. McDuff and D. Salamon, J-holomorphic Curves and Symplectic Topology, Colloquium Publications 52, Amer. Math. Soc., Providence, RI, 2004

[27] J. McKay, Graphs, singularities and finite groups, Proc. Symp. in Pure Math. 37 (1980), 183-186.

[28] I. Moerdijk, Orbifolds as groupoids: an introduction, in "Orbifolds in Mathematics and Physics", 205222, Edited by Adem et al., Contemporary Mathematics 310, Amer. Math. Soc., Providence, RI, 2002.

[29] D. A. Pronk, Groupoid representations for sheaves on orbifolds, Ph.D. Thesis, Utrecht 1995.

[30] M. Reid, La correspondance de McKay, Séminaire Bourbaki, Vol. 1999/2000, Astérisque 276 (2002), 53-72.

[31] I. Satake, The Gauss-Bonnet theorem for V-manifolds, J. Math. Soc. Japan 9 (1957), 464-492.

[32] G. Segal, Elliptic cohomology, Séminaire Bourbaki, Vol. 1987/1988, Astérisque 161/162 (1988), 187-201

[33] W. Thurston, The geometry and topology of three-manifolds, Princeton lecture notes.

[34] C. Vafa, Topological mirrors and quantum rings, Essays on mirror manifolds, 96-119, Internat. Press, Hong Kong, 1992.

[35] E. Witten, Elliptic genera and quantum field theory, Comm. Math. Phys.109 (1987), no. 4, 525-536

Current Address: Department of Mathematics and Statistics, University of Massachusetts at Amherst, Amherst, MA 01003. e-mail: wchen@math.umass.edu 